\title{
Regulation of septum formation by RHO4 GTPase signalling in Neurospora crassa
}

\author{
Dissertation \\ zur Erlangung des Doktorgrades \\ der Mathematisch-Naturwissenschaftlichen Fakultäten \\ der Georg-August-Universität zu Göttingen
}

vorgelegt von

Daniela Justa-Schuch

aus Quakenbrück

Göttingen 2010 
Die vorliegende Arbeit wurde von Januar 2006 bis März 2010 in der Abteilung Molekulare Mikrobiologie und Genetik unter Anleitung von Prof. Dr. Gerhard H. Braus am Institut für Mikrobiologie und Genetik der Georg-August-Universität zu Göttingen angefertigt.

D7

Referent:

Prof. G. H. Braus

Korreferent:

Prof. S. Pöggeler

Tag der mündlichen Prüfung: $\quad 30$. April 2010 
Teile dieser Arbeit wurden veröffentlicht in:

Justa-Schuch, D., Heilig, Y., Richthammer, C., Seiler, S. (2010) Septum formation is regulated by the RHO4-specific exchange factors BUD3 and RGF3 and by the landmark protein BUD4 in Neurospora crassa. Mol. Microbiol. 76: 220-235.

Si, H., Justa-Schuch, D., Seiler, S., Harris, SD. Regulation of septum formation by the Bud3Rho4-GTPase module in Aspergillus nidulans. Genetics. 185: 165-176. 


\section{Table of Contents}

$\begin{array}{ll}\text { Summary } & 1\end{array}$

Zusammenfassung 2

1. Introduction 3

$\begin{array}{lll}1.1 & \text { Cytokinesis } & 3\end{array}$

$\begin{array}{lll}\text { 1.2 Selection of the division site } & 5\end{array}$

1.2.1 Division site selection in Saccharomyces cerevisiae 5

1.2.2 Division site selection in Schizosaccharomyces pombe 6

$\begin{array}{lll}\text { 1.2.3 Division site selection in filamentous fungi } & 7\end{array}$

$\begin{array}{lll}1.3 & \text { Coordination of cytokinesis with the cell cycle } & 8\end{array}$

1.3.1 Coordination of cytokinesis with the cell cycle in S. cerevisiae $\quad 9$

1.3.2 Coordination of cytokinesis with the cell cycle in $S$. pombe 11

1.3.3 Coordination of cytokinesis with the cell cycle in filamentous fungi 12

1.4 Formation of the actomyosin ring, ring contraction and septum formation 14

1.4.1 Formation of the actomyosin ring, ring contraction and septum formation in $S$. cerevisiae

1.4.2 Formation of the actomyosin ring, ring contraction and septum formation in S. pombe

1.4.3 Formation of the actomyosin ring, ring contraction and septum formation in filamentous fungi

\subsection{Rho GTPases}

1.5.1 Rho GTPases and cytokinesis/septation 23

1.5.2 Rho4 GTPase family members and septation 24

1.6. Aims of this work $\quad 24$

2. Materials and methods 26

$\begin{array}{lll}2.1 & \text { Media and growth conditions }\end{array}$

$\begin{array}{lll}2.2 & \text { Plasmid construction } & 27\end{array}$

2.2.1 MYC-tagged overexpression constructs of $r g f-3$ and bud-3 27

2.2.2 GFP constructs for localization studies 27

2.2.3 Maltose binding protein (MBP) constructs for in vitro GEF assays 28

2.2.4 Deletion cassettes for the double knockout $\Delta r g f-3 ; \Delta b u d-3 \quad 29$

$\begin{array}{lll}2.2 .5 & \mathrm{DBF} 2^{\mathrm{T} 671 \mathrm{E}} \text { plasmid } & 30\end{array}$ 
2.3.1 E. coli

2.3.3 N. crassa

2.4 General molecular biological methods 36

2.4.1 Genomic DNA preparation and hybridisation 36

2.4.2 PCR 36

$\begin{array}{lll}2.4 .3 & \text { DNA isolation } & 37\end{array}$

$\begin{array}{ll}2.4 .4 & \text { Ligation and restriction digests } \\ 2.4 .5 & 37\end{array}$

2.4.5 DNA transformation in E. coli 37

$\begin{array}{lll}\text { 2.4.6 Sequence analysis } & 37\end{array}$

$\begin{array}{lll}2.5 & \text { Biochemical methods } & 38\end{array}$

2.5.1 Protein expression and purification in E. coli 38

$\begin{array}{ll}\text { 2.5.2 In vitro GEF assays } & 38\end{array}$

$\begin{array}{ll}\text { 2.5.3 Protein isolation and analysis } & 39\end{array}$

$\begin{array}{ll}\text { 2.5.4 Immunoprecipitation } & 40\end{array}$

$\begin{array}{lll}2.6 & \text { Microscopy } & 40\end{array}$

$\begin{array}{lll}2.6 .1 & \text { Fluorescence microscopy } & 41\end{array}$

3. Results $\quad 42$

3.1 Septation markers in Neurospora crassa 42

3.2 The two distinct exchange factors BUD3 and RGF3 control $\begin{array}{ll}\text { RHO4-dependent septum formation } & 44\end{array}$

3.3 Aspergillus nidulans AnBud3 acts as an AnRho4 GEF and can also stimulate $N$. crassa RHO4 activity $\quad \mathbf{5 0}$

3.4 BUD3 and RGF3 define two independent functions of RHO4 during septation

3.5 The two RHO4 modules BUD3-RHO4 and RGF3-RHO4 are acting prior to actomyosin ring formation

3.6 The localization of BUD4 at the cortex is independent of BUD3 and RGF3

3.7 CDC12-GFP and CLA4-GFP localize as constricting rings at forming septa 64 
4.2 The two RHO4 modules BUD3-RHO4 and RGF3-RHO4 are involved in septation

4.3 BUD3 functions in site selection and the actual septation process

4.4 The anillin-related protein BUD4 marks future septation sites

4.5 BUD3 and BUD4 do not seem to have a prominent role in polar growth

4.6 Expression of dominant-active RHO4 suppressed the septation defect of $\Delta$ bud-3 but not of $\Delta r g f-3$

4.7 BUD3-RHO4 and RGF3-RHO4 are required for the formation of the contractile actomyosin ring and act downstream of BUD4

4.8 Outlook

5. References

Acknowledgements 


\section{Summary}

Cytokinesis, the process in which a single cell is separated into two daughter cells, is highly conserved from plants to fungi and animals. Consistent with being key regulators of many cellular processes Rho GTPases have multiple, yet poorly-defined functions during cytokinesis. The Rho GTPase RHO4 in the euascomycete Neurospora crassa is essential for septation. By screening a $N$. crassa knockout collection for deletion mutants that phenocopy rho-4 defects (i.e. lack of septa, thin hyphae, slow growth, abnormal branching and cytoplasmic leakage), two strains defective in putative Rho guanine nucleotide exchange factors (GEFs) were identified. They were named BUD3 and RGF3 according to their homologues Bud3p and Rgf3p in budding and fission yeast, respectively. The function of these two proteins as RHO4-specific GEFs was determined by in vitro GEF assays. Likewise, AnBud3, the BUD3 homologue in Aspergillus nidulans, was identified as GEF of A. nidulans AnRho4 and N. crassa RHO4 in vitro, indicating that the Rho4-Bud3 module and its function during septation is conserved throughout the euascomycete lineage.

In vivo microscopy with GFP fusion proteins suggested that the two N. crassa GEFs and their target GTPase RHO4 function as two independent modules during septation with BUD3RHO4 acting in site selection and RGF3-RHO4 acting in the actual septation process.

Furthermore, the $N$. crassa homolog of the anillin-related protein BUD4 was determined as being required for septum initiation. Its deficiency leads to typical rho-4 defects. Localization of BUD4 as a cortical ring prior to septation initiation was independent of functional BUD3 or RGF3. These data position BUD4 upstream of both RHO4 functions in the septation process and make BUD4 a prime candidate for a cortical marker protein involved in the selection of future septation sites. The persistence of both BUD proteins and of RHO4 at the septal pore suggests additional functions of these proteins at mature septa. 


\section{Zusammenfassung}

Zytokinese bezeichnet den Prozess, bei dem eine einzelne Zelle in zwei Tochterzellen geteilt wird, und ist bei Eukaryoten hoch konserviert. Die Rho GTPasen sind in ihrer Funktion als Schlüsselregulatoren zahlreicher zellulärer Prozesse auch wesentlich an der Zellteilung beteiligt. Über die genauen Funktionen der Rho Proteine und deren Regulation in diesem Prozess war zu Beginn meiner Arbeit jedoch wenig bekannt. Die Rho GTPase RHO4 des Euascomyceten Neurospora crassa ist essentiell für die Septierung. Im Rahmen dieser Arbeit wurde die N. crassa Knockout-Stammsammlung nach Deletionsmutanten durchsucht, die dieselben Defekte zeigten wie die rho-4 Mutante, d.h. fehlende Septen, dünne Hyphen, abnormes Verzweigungsmuster, langsames Wachstum und zytoplasmatisches Auslaufen. Hierbei wurden die zwei Guaninnukleotid-Austauschfaktoren (GEFs) BUD3 und RGF3 identifiziert, die nach ihren Homologen Bud3p und Rgf3p in Saccharomyces cerevisiae und Schizosaccharomyces pombe benannt wurden. Mittels in vitro GEF-Assays wurde gezeigt, dass beide Proteine spezifisch die GDP/GTP-Austauschaktivität von RHO4 stimulieren. Auf gleiche Weise wurde das in Aspergillus nidulans vorkommende BUD3 Homolog AnBud3 als in vitro GDP/GTP-Austauschfaktor von $A$. nidulans AnRho4 und $N$. crassa RHO4 identifiziert. Diese Ergebnisse deuten daraufhin, dass es sich bei dem RHO4-BUD3 Modul um ein konserviertes Modul innerhalb der Euascomyceten handeln könnte.

In vivo Lokalisationsstudien zeigten, dass BUD3 und RGF3 wie die Rho GTPase RHO4 an Septen akkumulieren. Anschließende Untersuchungen zu Lokalisationsabhängigkeiten lassen vermuten, dass die beiden GEFs jeweils zusammen mit RHO4 als zwei voneinander unabhängige Module in der Septierung agieren. Das Modul BUD3-RHO4 scheint hierbei eher an der Festlegung der Septierungsstelle beteiligt zu sein, während RGF3-RHO4 seine Funktion womöglich in dem eigentlichen Septierungsprozess ausübt.

Neben den beiden GEFs wurde das Anillin-ähnliche Protein BUD4 als weiteres essentielles Protein für die Septierung in N. crassa identifiziert. Die bud-4 Deletionsmutante wies die oben beschriebenen rho-4 Defekte auf. BUD4 akkumulierte wie BUD3, RGF3 und RHO4 an Septen in vivo. Seine Lokalisation als kortikaler Ring an der zukünftigen Septierungsstelle, noch vor der eigentlichen Septenbildung, war unabhängig von funktionellem BUD3 oder RGF3. Dies legt den Schluss nahe, dass BUD4 oberhalb von beiden RHO4-Modulen als kortikales Markerprotein für die Septierungsstelle agiert. Da zudem BUD3 und BUD4 sowie RHO4 an der Pore vollständig ausgebildeter Septen verblieben, ist es möglich, dass diese Proteine dort zusätzliche Funktionen besitzen. 


\section{Introduction}

\subsection{Cytokinesis}

Cytokinesis is the process that separates a single cell into two daughter cells. It is essential for cell proliferation and requires the concerted action of many proteins that have to be tightly regulated in space and time (Eggert et al., 2006). The basic mechanisms of cytokinesis are conserved from plants to fungi and animals: at first the division site is selected, then the division machinery is assembled followed by cell separation. A further common feature is the coordination of these processes with the nuclear cycle (Fig.1; reviewed in Barr \& Gruneberg, 2007, Eggert et al., 2006, Field et al., 1999, Guertin et al., 2002, Pollard \& Wu, 2010).

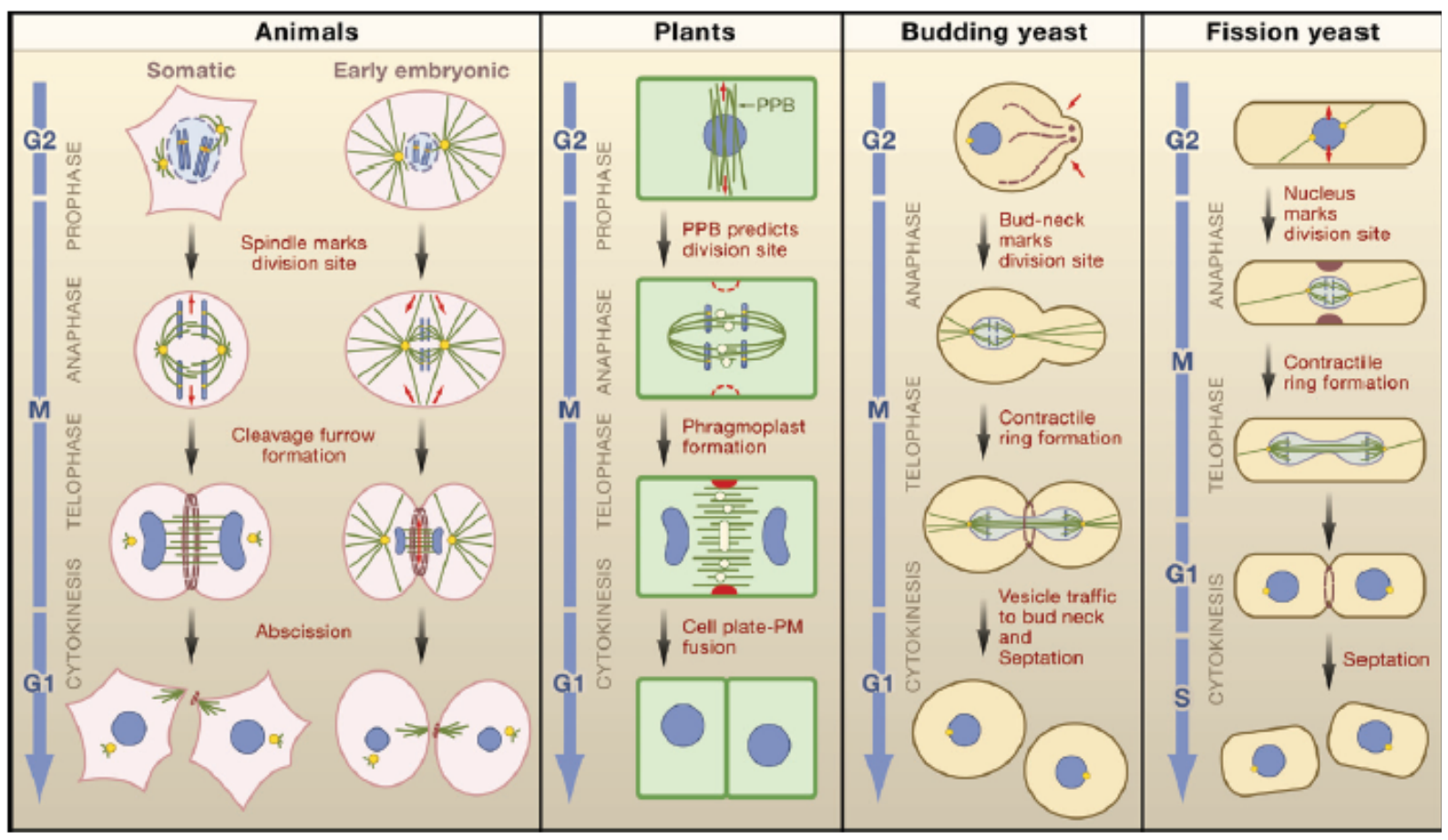

Fig. 1: Cytokinesis in animals, plants, and budding and fission yeast (Barr \& Gruneberg, 2007). The selection of the division site and cytokinesis relative to other key cell-cycle transitions is shown for animal cells, plants, and budding and fission yeast. While in animals the division site is determined by the position of the spindle midzone at the metaphase to anaphase transition, the division site in plants is marked by the preprophase band (an array of microtubules) as cells enter mitosis. In budding yeast the site of cell division is predetermined by the bud scar from the previous cell cycle and in fission yeast the site is selected by the position of the premitotic nucleus. Unlike in plants, which form a dense structure consisting of microtubuli and actin called phragmoplast for cell separation, fungi and animals make use of a contractile actomyosin ring to separate the cells. In plants vesicles accumulate and fuse at the division site after phragmoplast formation to form a new cell plate. Fungi synthesize a chitin-rich septum at the division site during actomyosin ring constriction. For further details please see text. Microtubules, green; actin and the actomyosin contractile ring, brown; kinetochores and centrosomes, yellow; chromosomes, blue.

A recent study revealed that also the protein composition of the cytokinesis complex is conserved across metazoan lineages (Eme et al., 2009). The authors of this study postulated 
that the present-day eukaryotic cytokinesis structures derive from the cell division machinery present in the last common ancestor of eukaryotes (Eme et al., 2009). Nevertheless, it varies how the different organisms position the division plane and how they perform cell separation (Fig.1; reviewed in Barr \& Gruneberg, 2007, Eggert et al., 2006, Field et al., 1999, Guertin et al., 2002, Oliferenko et al., 2009). While in animals the division site is determined by the position of the spindle midzone at the metaphase to anaphase transition and generally lies at the cell equator, the division site in plants is marked by an array of microtubules called preprophase band as cells enter mitosis. In budding yeast the site of cell division is predetermined by the bud scar from the previous cell cycle and is marked by landmark proteins prior to mitosis. In fission yeast the site is also selected prior to mitosis by the position of the premitotic nucleus. Unlike in plants, which form a dense structure consisting of microtubuli and actin called phragmoplast between the dividing nucleus for cell separation, fungi and animals make use of a contractile actomyosin ring to separate the cells (reviewed in Barr \& Gruneberg, 2007, Eggert et al., 2006, Field et al., 1999, Guertin et al., 2002, Oliferenko et al., 2009). In animals a cleavage furrow containing the actomyosin ring is formed at the division site. After furrowing is successfully completed, abscission takes place and two separated cells are generated. Fungi synthesize a chitin-rich septum at the division site during actomyosin ring constriction. This septum is eventually degraded in the unicellular yeasts Saccharomyces cerevisiae and Schizosaccharomyces pombe to obtain two separated cells. However, in filamentous fungi septation, which corresponds to cytokinesis, is only loosely coordinated with nuclear position and cell cycle (Gladfelter \& Berman, 2009), and the formed septum is not necessarily degraded to generate separated cells. Rather multicellular hyphae are formed that are partitioned through septa into hyphal units. In most filamentous fungi a small pore is retained in the septum allowing the controlled flow of cytoplasm and organelles between different compartments. The connection of hyphal units into multicellular structures is the basis for the morphological complexity achieved by filamentous fungi and thus for the success of the fungal kingdom (Gladfelter, 2006, Madhani \& Fink, 1998, Pringle $\&$ Taylor, 2002). Furthermore, increasing evidence suggests a more complex and dynamic regulation of septal functions in filamentous fungi than in yeasts. For example, the mature septum is a dynamic structure and organelles can be associated with it: well characterized are the Woronin bodies, which are peroxisomal derivatives that plug the septal pore upon injury of the hyphae (Jedd \& Chua, 2000) or the presence of microtubule organizing centers associated with septa (Veith et al., 2005). Furthermore, septation is also needed for certain 
steps of fungal development such as conidiation (production of asexual spores) and protoperithecia (female sexual structure) formation.

In the following similarities and differences between cytokinesis/septation in the two unicellular yeasts $S$. cerevisiae and $S$. pombe and in filamentous fungi will be described.

\subsection{Selection of the division site}

Unlike in animals, in fungi the division site is predetermined prior to mitosis. The mechanisms for determination of the cleavage site are highly species-specific. While in budding yeast the septation site is predetermined by the bud scar and is marked by a set of landmark proteins, in fission yeast the site is selected by the position of the premitotic nucleus. So far, not much is known about how filamentous fungi select the division site. In multiple fungal genomes homologues of the budding yeast landmark genes and of the fission yeast $M I D 1$, which is involved in providing the spatial cue for septation in this yeast, are present. This suggests that the corresponding proteins may have a conserved role in determining future division sites in the fungal kingdom. In addition, similar to the situation in fission yeast, the future septation site in the filamentous fungus $A$. nidulans seems to be coupled with nuclear position.

\subsubsection{Division site selection in Saccharomyces cerevisiae}

The yeast $S$. cerevisiae divides by budding. The site of cell division is pre-selected in the interphase $(\mathrm{G} 1 / \mathrm{S})$ by the assembly of landmark proteins at the cell cortex marking the future bud site. Where these landmark proteins assemble, in turn, depends on the used budding pattern and is predetermined by the position of the bud scar from the previous cell cycle. The budding yeast exhibit two different budding patterns depending on the mating type. While haploid cells of the mating types a or $\alpha$ show an axial budding pattern placing the new bud adjacent to its previous division site, diploid $\mathbf{a} / \alpha$ cells exhibit a bipolar budding pattern placing the new bud either proximal or distal to the site of previous cell division. In case of axial budding pattern the future bud-site is transiently marked by the landmark proteins Bud3p and Bud4p, that can interact with septins, the cell wall protein Ax12p/Bud10p and the haploid specific protein Axl1p (Casamayor \& Snyder, 2002, Gao et al., 2007, Lord et al., 
2002). Loss of any of these landmark proteins results in a bipolar budding pattern in haploid cells, whereas the budding pattern in diploids is not affected (Casamayor \& Snyder, 2002). To establish a bipolar budding pattern in diploid cells Bud8p and Bud9p are used as persistent markers to mark the poles distal and proximal to the last division site, respectively (Harkins et al., 2001, Schenkman et al., 2002, Zahner et al., 1996). The localization of Bud8p and Bud9p is, at least partially, dependent on the membrane proteins Rax1p and Rax $2 \mathrm{p}$, which can interact with both landmark proteins (Kang et al., 2004).

In both described budding patterns the landmark proteins help recruit a guanosine triphosphatase (GTPase) module consisting of the GTPase Bud1p, its GTPase activating protein (GAP) Bud2p and its guanine-nucleotide exchange factor (GEF) Bud5p to the future bud site. This module is essential for bud-site selection in yeast, deletion of either of these genes results in a randomized budding pattern in either cell-type (Bender \& Pringle, 1989, Chant et al., 1991, Chant \& Herskowitz, 1991, Kang et al., 2001, Park et al., 1997, Park et al., 1993, Park et al., 2002). After activation the GTPase Budlp recruits the small Rho (Ras homologous) GTPase Cdc42p and its GEF Cdc24p near the bud scar (Kozminski et al., 2003, Park et al., 1997, Zheng et al., 1995). It is likely that the bud site localization of the Bud1GTPase module is in turn dependent on $\mathrm{Cdc} 42 \mathrm{p}$ thereby establishing a positive feedback loop between both GTPase modules (Slaughter et al., 2009). The Cdc42-module is involved in growth and polarity establishment. Its activiation leads to the assembly of septins at the future bud, which form a ring there (Iwase et al., 2006). The septin ring serves to direct the formation of a new bud (Longtine et al., 1996). To ensure that a division site is not used twice, Rgalp, the GAP for Cdc42p, localizes to the bud site and inactivates local Cdc $42 p$ (Tong et al., 2007). Since the selection of the division site in budding yeast occurs early in the cell cycle, the formation of the spindle and its position has to be coordinated with the motherbud axis. The correct orientation of the spindle is coupled with the activation of the mitotic exit network (MEN; see section 1.3.1), so that in case of a failure cytokinesis is not performed (Lee et al., 1999, Miller et al., 1999, Schuyler \& Pellman, 2001, Sheeman et al., 2003, Yeh et al., 2000).

\subsubsection{Division site selection in Schizosaccharomyces pombe}

The fission yeast $S$. pombe grows by elongation at its tips and divides by medial fission generating two roughly equally sized daughter cells. Unlike in budding yeast, in which the division site is predetermined by the previous cell cycle, the division site in fission yeast is 
chosen in the late interphase prior to mitosis. It is dependent on the position of the premitotic nucleus (Paoletti \& Chang, 2000) and alterations in the localization of the nucleus until early mitosis lead to repositioning of the division site as well (Daga \& Chang, 2005, TolicNorrelykke et al., 2005). During cell growth, the position of the nucleus at the middle of the cell is maintained through opposing pushing forces generated by microtubules (Tran et al., 2001). Consistently, tubulin mutants often exhibit an aberrant localization of the division plane caused by mispositioning of the nucleus (Chang et al., 1996).

A key factor for defining the division plane by linking the position of the nucleus to actomyosin ring formation is the anillin-like protein Mid1p. Mid1p localization, in turn, is restricted to the middle of the cell by the position of the nucleus acting as positive cue (Daga $\&$ Chang, 2005), and by the polarity factors Pom1p, Tea1p and Tea4p, which localize to the cell ends and act as negative cue inhibiting cytokinesis there (Celton-Morizur et al., 2006, Huang et al., 2007, Padte et al., 2006). In interphase, Mid1p shuttles between the nucleus and the adjacent cell cortex with being predominantly accumulated in the nuclear compartment. The Cdr2p kinase anchors Mid1p at the medial cortex during interphase (Almonacid et al., 2009). Upon entry into mitosis triggered through the cell cycle kinase Cdc2p, Mid1p is released from the nucleus by phosphorylation through the Polo-family protein kinase Plo1p (Bahler et al., 1998). Once released, Mid1p forms a broad band consisting of many cortical nodes at the medial cortex defining the site for recruitment of actomyosin ring components (Paoletti \& Chang, 2000).

\subsubsection{Division site selection in filamentous fungi}

So far, not much is known about how filamentous fungi select the division site. The annotation of multiple fungal genomes revealed the presence of homologues of $B U D$ genes and/or of homologues of MIDl in the genomes of hemiascomycetes as well as of euascomycetes (Borkovich et al., 2004, Dietrich et al., 2004, Harris et al., 2009, Jones et al., 2004, Wortman et al., 2009). This suggests that the corresponding proteins may have a conserved role in determining future division sites in the fungal kingdom.

Until now, only the Bud4p homologue Int1p in the hemiascomycete Candida albicans, which can grow either as unicellular yeast or in a multicellular form, and the Bud3p homologue AgBud3p in the hemiascomycete Ashbya gossypii are functionally characterized (Gale et al., 2001, Wendland, 2003). Intlp is important for the axial budding pattern in C. albicans, as described for its homologue in the closely related $S$. cerevisiae. It colocalizes with the septin 
Cdc $3 p$ in a ring at the mother-bud neck of yeast and pseudohyphal cells. Under conditions that induce the formation of hyphae, both $\mathrm{Cdc} 3 p$ and Int $1 p$ localize to a ring distal to the junction of the mother cell and germ tube (Gale et al., 2001). AgBud3p in Ashbya acts like its S. cerevisiae homologue Bud3p as a landmark tagging future sites of septation. However, it is not involved in directing lateral branching. In addition to its landmark function, $\mathrm{AgBud} 3 \mathrm{p}$ is part of a scaffold that is involved in recruiting components of the contractile actomyosin ring to the septation site (Wendland, 2003). Thus, at least in closely related species of S. cerevisiae landmark homologues seem to have a conserved function in site selection.

In $S$. pombe the position of the division site is dependent on the localization of the premitotic nucleus (Paoletti \& Chang, 2000). In filamentous fungi septation seems to be only loosely coordinated with nuclear position (Gladfelter \& Berman, 2009). So far, the only known filamentous fungus, in which the future septation site is coupled with nuclear position, is the euascomycete Aspergillus nidulans (Kaminskyj \& Hamer, 1998, Wolkow et al., 1996). In this fungus septa are usually formed between pairs of daughter nuclei. In mutants with a disturbed nuclear distribution septa are distributed unevenly as well (Harris, 2001, Harris et al., 1994, Kaminskyj, 2000, Momany \& Hamer, 1997, Wolkow et al., 1996). However, how septation is coupled with the positioning of nuclei in this fungus is at present not known.

Furthermore, it has been speculated that the hyphal tip is involved in determining septal sites in A. nidulans and in A. gossypii (Dynesen \& Nielsen, 2003, Kaminskyj, 2000, Knechtle et al., 2003). In A. gossypii the growth speed of a hyphal tip in a young mycelium was shown to be decreased during lateral branch or septum formation (Knechtle et al., 2003). It is suggested that during this phase of decreased growth the hyphal tip serves as initiation point for new septa or lateral branches by positioning landmark proteins such as AgBud3p to this site (Knechtle et al., 2003, Walther \& Wendland, 2003, Wendland, 2003). Similarly, in $A$. nidulans the branching frequency was found to be increased when hyphal tip extension was reduced (Dynesen \& Nielsen, 2003). However, since these are very indirect data and since for example the hyphal tip growth speed was only occasionally and then weakly influenced by branching or septation events in later developmental stages in A. gossypii (Knechtle et al., 2003), further studies are necessary to verify this hypothesis.

\subsection{Coordination of cytokinesis with the cell cycle}

In general, successful cell division requires the temporal coordination of chromosome segregation with the constriction of the actomyosin ring and delivery of membrane and cell 
wall (in fungi) material. To ensure that mitotic exit and cytokinesis do not occur before the chromosomes are segregated properly, the onset of cytokinesis is tightly coupled to the cell cycle. In eukaryotes, the cell cycle is controlled by the activity of cyclin dependent kinases (CDKs), which, in turn, are regulated by cyclins and through phosphorylation and dephosphorylation events. CDKs are only active in a complex with their corresponding cyclin, whose concentration varies in cyclical fashion during the cell cycle. To exit from mitosis, the mitotic CDKs must be inactivated (reviewed in Doonan \& Kitsios, 2009). Pololike kinases also play a major regulatory role in cell cycle progression in eukaryotes, for example by regulating the activity of CDK-cyclin complexes. Amongst others, they promote the entry into mitosis, function at the spindle poles and promote the mitotic exit and cytokinesis (Archambault \& Glover, 2009).

Like in animals, in budding and fission yeasts, cytokinesis is tightly regulated in a cell-cycledependent manner. The assembly of the actomyosin ring is dependent on entry into mitosis in both yeasts. The temporal coordination of nuclear division and cytokinesis is mediated by the signal transduction networks SIN (septation initiation network) and MEN (mitotic exit network) in S. pombe and S. cerevisiae, respectively (see sections 1.3.1 and 1.3.2 and Fig. 2). SIN and MEN components are highly conserved and were also identified in the genomes of multiple filamentous fungi. However, whether SIN/MEN has an analogous function in filamentous fungi is at present not known.

\subsubsection{Coordination of cytokinesis with the cell cycle in $S$. cerevisiae}

In budding yeast the polo kinase Cdc5p and the mitotic exit network (MEN) network are involved in coordination of the mitotic exit with the onset of cytokinesis (Fig. 2A; Lippincott et al., 2001, Song \& Lee, 2001). During anaphase, Cdc5p activates the MEN signalling cascade, which regulates exit of mitosis by inactivation of the Cdc28-cyclin B complex and onset of cytokinesis. Central components of the MEN network are the GTPase Tem1p, the protein kinase Cdc15p and the nuclear Dbf2 related (NDR) kinase Dbf2p together with its associated subunit Mob1p (Fig. 2A; Bardin \& Amon, 2001, Luca et al., 2001). Activation of MEN through Cdc5p occurs by phosphorylating Bfa1p, which functions together with Bub2p as a bipartite GAP for Tem1p, resulting in inhibition of the GAP function. This, in turn, leads to activation of the GTPase Tem1p, which is the most upstream component of MEN (Hu et al., 2001, Simanis, 2003). Tem1p activation is followed by Cdc15p and subsequently Dbf2pMob1p complex activation (Asakawa et al., 2001, Bardin et al., 2000, Lee et al., 2001b, Mah 
et al., 2001). Furthermore, Cdc5p assists the release of the protein phosphatase Cdc $14 p$ from the nucleolus by phosphorylating Net1p (Yoshida et al., 2002). Net1p is a nucleolar inhibitor of Cdc14p, which forms a complex with Cdc14p in the nucleolus (Yoshida et al., 2002). Subsequently, Cdc14p is phosphorylated through the active Dbf2p-Mob1p complex and is thereby released into the cytoplasm (Mohl et al., 2009). Once in the cytoplasm, Cdc14p reverses Cdk phosphorylation events to facilitate mitotic exit (Clifford et al., 2008). For example, it dephosphorylates Cdh1p, which is an anaphase promoting complex (APC) activator protein. This allows Cdh1p to interact with the $\mathrm{APC} / \mathrm{C}$ and to drive cyclin degradation leading to exit from mitosis (Simanis, 2003).

As mentioned above, Cdc5p and the MEN network do not only control cyclin degradation and thus mitotic exit, but also play a role in cytokinesis. This is supported by following observations: MEN mutants are available, in which mitotic exit still occurs, but cytokinesis is inhibited (Jimenez et al., 1998). In addition, cells with mutations in Net1p that bypass the requirement for MEN in cyclin degradation still show defects in cytokinesis (Lippincott et al., 2001). Similarly, mitotic exit in cells with mutations in the MEN components can be induced by overexpression of the CDK inhibitor Sic1p, but cytokinesis is still disturbed (Hwa Lim et $a l ., 2003)$. Furthermore, the polo-box of Cdc5p was shown to interact with the septins Cdc11p and Cdc12p, whose presence at the bud neck is known to be necessary for bud growth (Song \& Lee, 2001). Components of the MEN, such as the GTPase Tem1p, have also been implicated in the regulation of septin and actomyosin ring dynamics (Lee et al., 2001a, Lippincott et al., 2001, Simanis, 2003). 


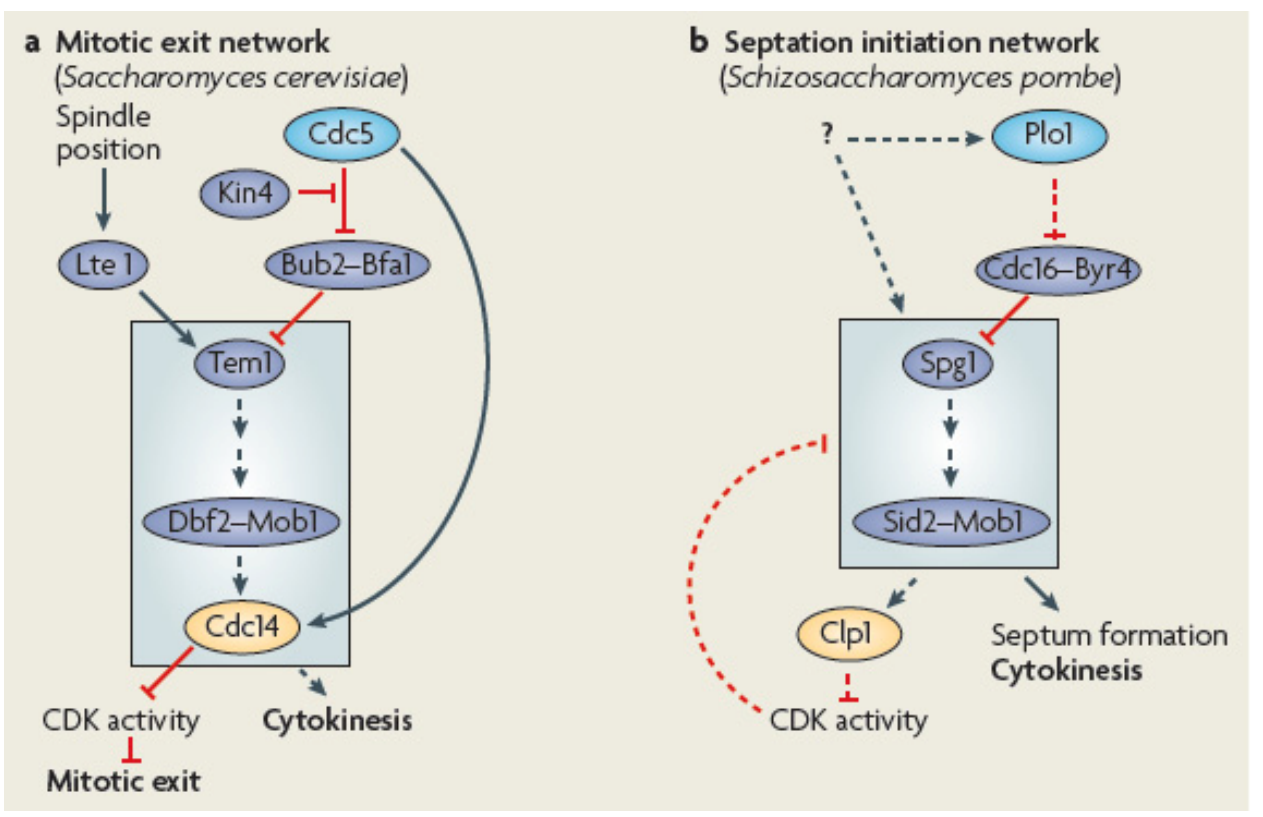

Fig. 2: The MEN (a) and SIN (b) networks in budding and fission yeast (modified from Archambault \& Glover, 2009). (a) In $S$. cerevisiae the MEN contributes to mitotic exit. The Tem1p GTPase is localized on spindle pole bodies and is activated by the GEF Lte1p. Because Lte1p is localized to the bud cortex, the MEN is activated following anaphase and the proper positioning of the spindle through the mother bud neck. In addition, the polo kinase Cdc5p promotes MEN activation by inhibiting the Bub2p-Bfalp GAP complex that accelerates GTP hydrolysis by Tem1p. Bfa1p is rendered insensitive to Cdc5p through phosphorylation by the kinase Kin $4 p$, thereby inhibiting mitotic exit. A signalling cascade that involves several kinases downstream of Tem1p, and that includes the Dbf2p-Mob1p complex (other components are not shown), leads to the release of the Cdc14p phosphatase from nucleolar sequestration. Once in the cytoplasm, Cdc14p reverses Cdk phosphorylation events to facilitate mitotic exit. Cdc5p also function in a network that leads to an initial, earlier burst of Cdc14 release that potentiates MEN activation. Cdc5p and MEN do not only control mitotic exit, but also play a role in cytokinesis. (b) The SIN network in fission yeast is molecularly similar to the MEN. The Spg1p GTPase is kept in its GDP-bound form by the Cdc16p-Byr4p GAP. In late mitosis, the polo kinase Plo1p is thought to activate the SIN, possibly by inhibiting the Spg1p GAP. This leads to the activation of the Sid2p-Mob1p kinase complex, which triggers the assembly of the division septum for cytokinesis. The SIN also potentiates the nucleolar release of the Cdc14p-like phosphatase Clplp in late mitosis, which contributes to inactivate CDK activity, further facilitating SIN signalling. Plo1p also acts earlier in the cell cycle by promoting the nuclear export of Mid1p. Dashed lines indicate indirect or hypothetical connections.

\subsubsection{Coordination of cytokinesis with the cell cycle in $S$. pombe}

As mentioned above, in fission yeast cytokinesis is tightly coupled with the nuclear cycle. While the spatial cue for cytokinesis is provided by Mid1p, the temporal coordination of mitosis and cytokinesis is ensured by a signalling cascade known as the septation initiation network (SIN) (Fig. 2B; Hachet \& Simanis, 2008). The SIN signalling cascade is analogous to the MEN network in budding yeast (Fig. 2; Bardin \& Amon, 2001, Krapp \& Simanis, 2008). The only SIN components having no clear counterpart in MEN are the kinase Sid1p and its regulatory subunit $\mathrm{Cdc} 14 \mathrm{p}$. This additional kinase functions downstream of the kinase Cdc7p, the homologue of $S$. cerevisiae Cdc15p, and activates the Sid2p-Mob1p complex, which is homologous to the Dbf2p-Mob1p complex in S. cerevisiae. Similar to the situation in 
budding yeast, the Polo-like kinase Plo1p is thought to activate the SIN, possibly by inhibiting the GAP of the GTPase Spg1p, the orthologue of Tem1p (Archambault \& Glover, 2009). This results in the activation of the most downstream kinase Sid2p, which in turn phosphorylates the protein phosphatase Clp1p (Chen et al., 2008, Krapp \& Simanis, 2008). Clp1p is the homologue of Cdc14p in $S$. cerevisiae and like its homologue Clp1p is sequestered in the nucleolus in interphase (Clifford et al., 2008). Once phosphorylated by Sid2p in late mitosis, Clp1p is released from the nucleolus and maintained into the cytoplasm by binding to the 143-3 protein $\operatorname{Rad} 24 p$ (Chen et al., 2008, Mishra et al., 2005). Similar to the situation in budding yeast, the cytoplasmic retention of Clp1p promotes cytokinesis by dephosphorylating substrates of CDKs (Clifford et al., 2008). At the end of mitosis SIN triggers contraction of the actomyosin ring and formation of the division septum (Jin et al., 2006, Mishra et al., 2004). While inactivation of the SIN results in multinucleated cells that do not perform cytokinesis, SIN hyperactivation leads to multiseptated cells (Fankhauser \& Simanis, 1994, Minet et al., 1979, Ohkura et al., 1995, Roberts-Galbraith \& Gould, 2008, Schmidt et al., 1997). Furthermore, SIN is also involved in completion of the assembly of the contractile actomyosin ring (CAR) by controlling hypophosphorylation and recruitment of the $\mathrm{PCH}$ protein Cdc15p to the CAR (Hachet \& Simanis, 2008). Since the formation of actomyosin structures and the CAR in the absence of Mid1p activity are dependent on SIN function, it was concluded that the SIN and Mid1p orchestrate CAR assembly (Hachet \& Simanis, 2008).

\subsubsection{Coordination of cytokinesis with the cell cycle in filamentous fungi}

In filamentous fungi, septation seems to be only loosely coordinated with the cell cycle (Gladfelter \& Berman, 2009). Interestingly, components of SIN and MEN, which coordinate cell cycle and cytokinesis in fission and budding yeast, respectively, are also conserved in filamentous fungi. Whether the SIN/MEN functions analogously in filamentous fungi or whether its function has (partly) changed e.g. due to morphological differences, is at present unclear.

So far, there are SIN/MEN components functionally characterized in the euascomycetes $A$. nidulans and $N$. crassa and in the hemiascomycete $C$. albicans. All of them were described to be required for cytokinesis/septation in the respective fungus.

In C. albicans a homologue of the most downstream kinase ScDbf2p is present. Here, CaDbf2 is an essential protein and has several functions during mitotic exit. It is necessary for actomyosin ring contraction and cytokinesis. Additionally, $\mathrm{CaDbf} 2$ also regulates mitotic spindle organization and nuclear segregation. Furthermore, it is required for proper hyphal 
morphogenesis and septum assembly under hypha-inducing conditions (Gonzalez-Novo et al., 2009a).

In A. nidulans gene homologues of most of the SIN components are present in the genome (Kim et al., 2009). Homologues of the three SIN network kinases were identified with SepH being the most upstream kinase, SepL the central kinase and SidB the most downstream kinase. Moreover, SepM and MobA were described to function as co-factors for SepL and SidB, respectively (Kim et al., 2006, Kim et al., 2009). In addition, a homologue of the Spg1p/Tem1p GTPase, named AsgA, was identified in an expressed sequence tag (EST) database (Bruno et al., 2001). The SIN kinases SepH and SidB and the SidB co-factor MobA are essential for septation in Aspergillus (Bruno et al., 2001, Kim et al., 2006). This suggests that a signalling network similar to the SIN in fission yeast may regulate septation in this fungus. SepH, the orthologue of $S$. pombe Cdc $7 \mathrm{p}$, is required for the assembly of the septal band in response to mitotic signals and thus functions upstream of actin ring formation (Bruno et al., 2001, Harris, 2001). The localization of the septin AspB and the formin SepA to the septation site was shown to be dependent on SepH (Sharpless \& Harris, 2002, Westfall \& Momany, 2002). Moreover, MobA and SidB are required for the assembly of the CAR (Kim et al., 2006). Like its fission yeast counterparts Sid2p and Mob1p, SidB and MobA localize to the spindle pole bodies and later to the septation site (Kim et al., 2006). Interestingly, unlike in fission yeast, the SidB and MobA signal contracts along with the actomyosin ring (Kim et al., 2006).

Consistent with the presence of a signalling network resembling the SIN in S. pombe, septation in A. nidulans was described as being dependent on mitosis (Westfall \& Momany, 2002, Wolkow et al., 1996). However, the first three rounds of nuclear division are not accompanied by septation until cells reach an appropriate size/volume (Harris et al., 1994, Wolkow et al., 1996). In addition, due to the fact that the hyphal tip and the hyphal compartments are multinucleate, not all of the individual mitotic events can trigger septation. Taken together, the SIN network in A. nidulans may be involved in coordinating the septation process with the cell cycle as described for its yeast counterparts. However, since not each mitosis is followed by septation, the coordination of both processes in A. nidulans likely occurs in a different way than in both yeasts.

Unlike in A. nidulans, in N. crassa there is no obvious coordination between nuclear division and cytokinesis. Nevertheless, homologues of the most downstream MEN/SIN kinase complex Dbf2p-Mob1p and Sid2p-Mob1p in S. cerevisiae and S. pombe, respectively, are present and were named DBF2 and MOB1. Deletion of either gene results in an aseptate 
phenotype implicating a function of these genes in septum formation (Dvash et al., 2009, Maerz et al., 2009).

\subsection{Formation of the actomyosin ring, ring contraction and septum formation}

As described above septum formation occurs at a pre-determined place and is, in general, coordinated with the nuclear cycle. In fungi it has been shown that a contractile actomyosin ring, which consists of filamentous actin (F-actin), type II myosin, and several other proteins, is formed at the division site. The formation of this ring is a pre-requisite for septation, and its constriction generates the forces necessary for the division of a mother cell into two daughter cells. During the constriction of the ring membrane and septal cell wall material are deposited centripetally. However, while the basic composition of the actomyosin ring is very similar in all fungi, the assembly order of the components into the ring is different. These differences may be explained by species-specific features.

\subsubsection{Formation of the actomyosin ring, ring contraction and septum formation in S. cerevisiae}

In budding yeast the division site is defined by landmark proteins and coordination of mitosis with cytokinesis occurs through the MEN network (see sections 1.2.1 and 1.3.1). The first proteins that assemble at the bud neck are the septins. The assembly of septins is initiated by the Cdc42-GTPase module in the early interphase. Septins are GTP-binding, filament forming proteins that are conserved from yeast to human. In $S$. cerevisiae there are seven septins present. While Cdc3p, Cdc10p, Cdc11p, Cdc12p and Shs1p/Sep $7 p$ are vegetative septins and their deletion results in complete block of mitosis, Spr3p and Spr28p are expressed exclusively during sporulation. All five vegetative septins localize to the bud neck in an interdependent manner and form a ring at the bud site through which subsequently the bud emerges (Gladfelter et al., 2001, Iwase et al., 2006). The p21-activated kinase (PAK)-like kinase Cla $4 \mathrm{p}$, an effector protein of $\mathrm{Cdc} 42 \mathrm{p}$, was shown to be required for the correct localization and/or assembly of the septin ring (Schmidt et al., 2003). Septins act as scaffolds for other proteins involved in septum formation. They recruit the only myosin II heavy chain Myo1p and its regulatory chain Mlc2p together with the formin Bnr1p to the bud neck 
forming a ring there (Bi et al., 1998, Kamei et al., 1998, Lippincott \& Li, 1998b). In S phase the myosin II essential light chain Mlc1p and in S/G2 phase the PCH protein Hoflp/Cyk2p are recruited to the division site (reviewed in Balasubramanian et al., 2004). Localization of Hoflp is dependent on septins, but not on Myolp, and its deletion results in deficiency in cytokinesis. Furthermore Bnrlp and Hoflp interact with each other in a Rho4p-GTPase dependent manner (Kamei et al., 1998, Vallen et al., 2000). It is suggested that Hof1p functions as an adapter linking the primary septum synthesis machinery to the actomyosin system (Vallen et al., 2000). Subsequently, in G2/M phase the IQGAP protein Iqg1p/Cyk1p is localized to the division site through the interaction with Mlc1p (Shannon \& Li, 2000). Iqg1p plays a direct role in F-actin recruitment and is required for the assembly of actin filaments to the ring. Interestingly, Iqg1p was also found to interact in vitro with Tem1p, the MEN GTPase, which is required for mitotic exit (Shannon \& Li, 1999, Shannon \& Li, 2000). Together with Iqglp the second formin present in this yeast, Bnilp, is recruited to the bud neck. Formins are known to be able to nucleate actin filaments. Consistently, Bnilp is, together with Bnrlp, required for actin ring formation and their activity, in turn, is regulated by the Rho GTPase Rholp (Pruyne et al., 2002, Sagot et al., 2002, Tolliday et al., 2002, Vallen et al., 2000). Interestingly, Rholp activation is cell-cycle dependent. It is activated by the CDK-cyclin complex Cdc28p-Cln2p in the interphase at the G1/S transition in a process mediated by its GEF Tus1p (Kono et al., 2008). In late anaphase the actomyosin ring forms and Cyk3p, a further protein important for cytokinesis potentially acting downstream of Iqg1p, is recruited to the ring (Korinek et al., 2000). Contraction of the actomyosin ring is followed closely by the centripetal growth of the septum (Bi et al., 1998, Lippincott \& Li, 1998a). Therefore both processes need to be temporarily and spatially coordinated. Septins, amongst others, are involved in coordination of both processes. They are not only required for targeting actomyosin ring components to the bud neck, but they also recruit septal components such as the chitin synthases Chs $2 p$, which forms the primary septum, and Chs $3 p$, which synthesizes the chitin ring and $\sim 90 \%$ of cellular chitin (Chuang \& Schekman, 1996, Orlean, 1987) to the bud neck (DeMarini et al., 1997, Roh et al., 2002). The proteins Hoflp, Cyk3p and Inn1p seem to be involved in septum formation (Balasubramanian et al., 1998, Jendretzki et al., 2009, Kamei et al., 1998, Korinek et al., 2000, Lippincott \& Li, 1998b, Lippincott \& Li, 2000, Nishihama et al., 2009, Sanchez-Diaz et al., 2008, Vallen et al., 2000). Inn1p couples ring contraction to membrane ingression and induces, in cooperation with Cyk3p, the formation of the primary septum through activating the chitin synthase Chs $2 p$ (Nishihama et al., 2009, Sanchez-Diaz et al., 2008). Following that, a secondary septum is 
deposited around the primary septum from both the mother and the daughter cell (reviewed in Yeong, 2005). To separate mother and daughter cell from each other the chitinase Cts1p degrades the primary septum at the neck. In addition, glucanases function to hydrolyse the secondary septum and/or the surrounding cell wall holding mother and daughter cell together (reviewed in Yeong, 2005). Moreover, since septum formation is often slow and asymmetric in mutants lacking actomyosin contraction, there could be interplay between the actomyosin ring and the deposition of the septum material (Vallen et al., 2000).

Interestingly, whereas in animals and in fission yeast the formation of the contractile actomyosin ring is essential for cell viability, in budding yeast viable strains exist lacking a functional ring that are able to produce separated cells. Cells lacking the contractile ring appear to divide by the formation of the septum. This is probably due to the narrowness of the bud neck $(\sim 1 \mu \mathrm{m})$ (Bi et al., 1998). Cyk3p, Hoflp and Inn1p are involved in this alternative, actomyosin ring independent cytokinesis pathway with Cyk3p being a key component (Vallen et al., 2000). This was supported by studies showing that $C Y K 3$ suppresses the requirement for Iqg1p in viability and cytokinesis without restoring the actin ring and that overexpression of CYK3 leads to an actomyosin ring independent recruitment of Inn1p to the bud neck (Jendretzki et al., 2009, Korinek et al., 2000).

\subsubsection{Formation of the actomyosin ring, ring contraction and septum formation in $S$. pombe}

In fission yeast the spatial cue for cytokinesis is provided by the anillin-related protein Mid1p and the temporal coordination of mitosis and cytokinesis is ensured by the SIN network (see sections 1.2.2 and 1.3.2). The assembly of the contractile actomyosin ring occurs by the ordered recruitment of ring components (Wu et al., 2003). As one of the earliest markers Mid1p accumulates prior to spindle pole body (SPB) separation at the future division site and forms a broad cortical band there that defines the site for recruitment of ring components (Paoletti \& Chang, 2000, Sawin, 2007, Wu et al., 2003). Subsequently, the heavy chain of type II myosin Myo2p interacts with Mid1p and is anchored at the medial cortex (Motegi et al., 2004) followed by the two myosin light chains Cdc4p and Rlc1p (Le Goff et al., 2000, McCollum et al., 1995). Afterwards, the IQGAP-related protein Rng2p, the PCH domain protein $\mathrm{Cdc} 15 \mathrm{p}$ and the formin $\mathrm{Cdc} 12 \mathrm{p}$, which all are regulators of the actin cytoskeleton, join the broad band (Chang et al., 1997, Eng et al., 1998, Fankhauser et al., 1995, Wu et al., 2003). Cdc15p and Cdc12p interact with each other and recruit other actin binding proteins to 
the site to induce actin polymerization at the actomyosin ring (Carnahan \& Gould, 2003, Kovar et al., 2003). Just prior to anaphase, the band of proteins condenses into a contractile ring including actin, the tropomyosin Cdc8p (Balasubramanian et al., 1992), and the $\alpha$-actinin Ain1p (Wu et al., 2001). During anaphase B, the actin capping protein Acp2p (Kovar et al., 2005, Nakano et al., 2001, Wu et al., 2003) and the unconventional myosin-II Myp2p (Bezanilla et al., 1997) join the ring followed by the septin Spn1p (Longtine et al., 1996). Afterwards, contraction of the ring begins, whereupon Mid1p, Acp2p and Spn1p do not constrict with (Wu et al., 2003). Furthermore, the profilin Cdc3p (Balasubramanian et al., 1994) and the myosin assembly factor Rng3p (Balasubramanian et al., 1998) are also essential for the assembly and/or maintenance of the actomyosin ring (Carnahan \& Gould, 2003). As mentioned above, the formation of the contractile actomyosin ring is coupled to the cell cycle through the SIN network. Furthermore, SIN also regulates the formation of the division septum after the actomyosin ring is assembled. The secretion of new membranes and the assembly of the division septum occur in concert with actomyosin ring constriction. The actomyosin ring was shown to serve as a spatial cue for localization of Cps1p, a septumsynthesizing 1,3- $\beta$-glucan synthase. The temporal coordination of Cps1p localization within the cell cycle, however, was ensured by the SIN network (Liu et al., 2002). After the primary and the secondary septum were formed, cell separation occurs by enzymatic degradation of the primary septum. In fission yeast, the Rho GTPase Rho4p is involved in cell separation by targeting glucanases to the primary septum (Nakano et al., 2003, Santos et al., 2003, Santos et al., 2005). In addition, septins are required for this step (Longtine et al., 1996). Four of the seven identified septins localize to the division plane after the actomyosin ring is formed (Longtine et al., 1996). Mid2p, the second anillin-homologue present in fission yeast, was found to organize the septin ring during late mitosis promoting cell separation (Berlin et al., 2003, Tasto et al., 2003). Thus, unlike in budding yeast septins act late in cytokinesis serving as positional markers to target the secretory vesicles for dissolution of the septum. These functional differences of the septins in both yeasts are also reflected in the fact that septins are, unlike in S. cerevisiae, not essential for cell viability in $S$. pombe (reviewed in GonzalezNovo et al., 2009b). 


\subsubsection{Formation of the actomyosin ring, ring contraction and septum formation in filamentous fungi}

Filamentous fungi also form a contractile actomyosin ring (CAR) to execute cytokinesis and deposit membrane and septal cell wall material centripetally to the septation site during ring constriction. Thus, components of the ring, proteins involved in ring assembly such as formins, IQGAP related proteins, actin-binding proteins and $\mathrm{PCH}$ proteins, and proteins required for the formation of the septum are also conserved within filamentous fungi. So far, functional characterizations of septation components in these fungi suggest that the basic functions of these proteins are conserved within the fungal kingdom. However, the assembly order of the components to the septation site varies and minor functional differences exist, probably due to species-specific features. Until now the number of functionally characterized proteins involved in ring assembly, ring constrictions and septum formation in filamentous fungi is low.

Formins, which have essential roles in remodelling the actin and microtubule cytoskeleton (reviewed in Chesarone et al., 2010), seem to have a conserved role in septation. In both yeasts formins are required for actin ring formation (Chang et al., 1997, Tolliday et al., 2002). This is also true for the formin SepA present in A. nidulans (Harris et al., 1997, Harris et al., 1994, Sharpless \& Harris, 2002). It colocalizes with actin at septation sites forming a ring there that constricts upon septum formation (Sharpless \& Harris, 2002). While Cdc12p in fission yeast also forms constricting rings (Chang et al., 1997, Wu et al., 2003), this was not observed for Bnilp in budding yeast (Ozaki-Kuroda et al., 2001). AgBnr2p, one of the three formins present in A. gossypii, also localizes at septa suggesting a role for this protein in septation (Schmitz et al., 2006). In C. albicans the formin CaBnr1 forms a ring at the bud neck. This ring persists there throughout the cell-cycle until the end of cytokinesis when the CaBnr1 ring split into two rings (Li et al., 2008).

In fungi, septins are involved in cytokinesis acting as scaffolds or barriers to demarcate local compartments. However, when and to what extend they function in this process varies (Gonzalez-Novo et al., 2009b). AspB, the only essential septin in Aspergillus, is important for cellular division, branching, and conidiation and localizes to the division site as a single ring (Momany \& Hamer, 1997, Westfall \& Momany, 2002). Like Spnlp in S. pombe AspB is recruited to the septation site after formation of the actin ring (Westfall \& Momany, 2002, Wu et al., 2003). Moreover, AspB is unable to form rings in sepA mutants indicating that the presence of the formin is needed for its proper localization (Westfall \& Momany, 2002). The AspB ring does not constrict upon septum formation, but splits into a double ring flanking the 
septum (Westfall \& Momany, 2002). This ring splitting was also observed in S. cerevisiae and S. pombe (Dobbelaere \& Barral, 2004, Wu et al., 2003). After septum completion the basal AspB ring together with the actin and SepA ring disappears, whereas the apical AspB ring persists (Sharpless \& Harris, 2002, Westfall \& Momany, 2002). Consistent with the hypothesis that septation in Aspergillus is coordinated with the cell cycle, the SIN kinase SepH mentioned above was shown to be required for the localization of the septin AspB and the formin SepA to the site of septation (Sharpless \& Harris, 2002, Westfall \& Momany, 2002). AspA and AspC, two other septins in Aspergillus, are also involved in, but not required for septation (Lindsey et al., 2010, Momany et al., 2001). In A. gossypii the septin homologues AgCdc3, AgSep7, AgCdc11 and $\mathrm{AgCdc} 12$ are not essential, but required for selection and organization of the septation site (Gladfelter et al., 2005, Helfer \& Gladfelter, 2006, Kaufmann \& Philippsen, 2009). In this fungus septation generally occurs at the base of branches (Walther \& Wendland, 2003). Septins were shown to assemble into discontinuous rings composed of discrete "bars" or filaments at these branch sites and to locally promote mitosis there (Helfer \& Gladfelter, 2006). This suggests that septins act early, prior to mitosis, in the septation process in Ashbya as also described in the closely related budding yeast (Helfer \& Gladfelter, 2006). In C. albicans, the non-essential septin CaCdc11 shows the same localization pattern in yeast cells as Cdc11p in S. cerevisiae suggesting a similar function for this protein during cytokinesis in this fungus (Gonzalez-Novo et al., 2004, Sudbery, 2001, Warenda \& Konopka, 2002). In developing hyphae the septin CaCdc11 forms a ring at the neck of the germ tube (Sudbery, 2001) and in hyphal cells septins establish a nuclear division plane (Gladfelter et al., 2005).

Furthermore, IQGAP homologues were identified and functionally characterized in the fungi Ashbya and Candida. In general, IQGAP proteins are important regulators of the actin cytoskeleton and are involved in cytokinesis (reviewed in Brandt \& Grosse, 2007). Like their counterparts Iqg1p and Rng2p in budding and fission yeast, respectively, AgCyk1 and CaIqg1 are essential for actomyosin ring formation (Eng et al., 1998, Li et al., 2008, Lippincott \& Li, 1998b, Shannon \& Li, 2000, Wendland \& Philippsen, 2002). In Ashbya AgCyk1 localizes as a ring to future septation sites prior to chitin deposition and is essential for septum formation (Wendland \& Philippsen, 2002). Its localization, in turn, is restricted to septation sites by the landmark protein AgBud3 described above (Wendland, 2003). Interestingly, in S. cerevisiae Iqg1p was shown to be required for proper Bud4p localization (Osman et al., 2002). In Candida CaIqg1 localizes to the bud neck in yeast cells and forms a constricting ring there (Li et al., 2008). Unlike its counterpart in budding yeast CaIqg1 interacts with the formins 
CaBnil and CaBnrl that act as actin-nucleating proteins (Li et al., 2008, Tolliday et al., 2002). However, in fission yeast a genetic interaction between the IQGAP Rng $2 p$ and the formin Cdc12p was described (Eng et al., 1998). Interestingly, CaIqg1 is regulated by phosphorylation through the cyclin dependent kinase Cdc28 thereby coordinating cytokinesis with the cell cycle (Li et al., 2008).

In Ashbya, the PCH protein AgHof1 was shown to be essential for actin ring and septum formation (Kaufmann \& Philippsen, 2009). This is different to the situation in budding yeast, in which the disruption of Hoflp does not affect the assembly of the actomyosin ring but results in disassembly of the ring during contraction (Lippincott \& Li, 1998a). In S. pombe the $\mathrm{PCH}$ protein $\mathrm{Cdc} 15 \mathrm{p}$ was described as being essential for the maintenance of the actomyosin ring in late mitosis (Wachtler et al., 2006). Localization studies revealed that AgHof1 together with the type II myosin AgMyo1, AgBud3 and AgCyk1 form collars of cortical bars already adjacent at hyphal tips, thereby marking septation sites in A. gossypii (Kaufmann \& Philippsen, 2009). Subsequently, during hyphal elongation, bar-to-ring transition takes place and the proteins mentioned above localize as rings. This bar-to-ring transition of protein localization is a pre-requisite for actin ring assembly and is dependent on AgHofl and AgCyk1 but not on AgMyo1 (Kaufmann \& Philippsen, 2009). Up to several hours after site selection, the actin ring contracts, cytokinesis takes place and the septum is formed (Kaufmann \& Philippsen, 2009). Interestingly, in S. pombe Cdc15p and Rng2p and further proteins involved in ring formation localize prior to ring formation in nodes, which are precursors of the ring (reviewed in Pollard \& $\mathrm{Wu}, 2010$ ).

In yeast, the PAK-like protein kinase Cla4p is a downstream effector protein of the Cdc $42 p$ GTPase-module and thus, is involved in budding and cytokinesis (Cvrckova et al., 1995, Versele \& Thorner, 2004). Its counterpart in Ashbya, AgCla4 was found to be involved in septation, because its deletion results in mutants that are severely impaired in actin and chitin ring formation (Ayad-Durieux et al., 2000). In addition, a homologue of the Cdc42p-module could also be identified in the Ashbya genome (Wendland \& Philippsen, 2001).

Recently, the actinin-like protein ActA in Aspergillus was shown to localize as a set of double rings embracing the septum site. acnA deletion mutants exhibited defects in septation and conidiation indicating a role of AcnA in actin accumulation during septum formation. Taken together AcnA is assumed to function as an adaptor or an anchor protein for the actin ring (Wang et al., 2009). Furthermore, deposition of septal wall material is tightly coupled to the assembly and constriction of the CAR in A. nidulans (Momany \& Hamer, 1997), as described in baker's and fission yeast. 


\subsection{Rho GTPases}

Rho (as homologous) GTPases belong to the Ras superfamily of small guanosine triphosphatases (GTPases) and are highly conserved throughout eukaryotes. They are key regulators of many extracellular-stimulus-mediated cellular processes, such as regulation of actin and microtubule dynamics, cell cycle progression, gene expression and cell polarity, through their ability to bind to numerous downstream effectors in their active state. Rho proteins act as molecular switches and cycle between an active GTP-bound and an inactive GDP-bound conformational state. When active, Rho GTPases are associated with the plasma membrane and can interact with downstream effector proteins, whereas, upon inactivation, they are sequestrated to the cytoplasm (Fig. 3; for reviews see Buchsbaum, 2007, EtienneManneville \& Hall, 2002, Hall, 2005, Wennerberg et al., 2005). In budding yeast as well as in fission yeast six Rho GTPases named Rho1p to Rho5p and Cdc42p are described (reviewed in Levin, 2005, Nakano et al., 2003). In Neurospora crassa six genes were identified to encode Rho proteins and their corresponding proteins were named RHO1 to RHO4, RAC and CDC42 (Borkovich et al., 2004).

With being involved in so many important cellular processes, Rho GTPases are, in turn, tightly regulated (Fig. 3). As activators function the guanine nucleotide-exchange factors (GEFs). They catalyse the exchange of GDP for GTP in vivo by binding to Rho proteins via their GEF domain (also called Dbl homology domain) and thereby stabilizing the nucleotidefree status of the Rho proteins (reviewed in Rossman et al., 2005). GTPase-activating proteins (GAPs) suppress Rho activity by enhancing the low intrinsic GTPase activity of Rho proteins so that bound GTP is hydrolysed (for review see Tcherkezian \& Lamarche-Vane, 2007). Further regulators are the guanine nucleotide-dissociation inhibitors (GDIs). They form complexes with inactive GDP-bound Rho proteins to dissociate them from the membrane and sequester them in a cytoplasmic pool of inactive GTPases (reviewed in DerMardirossian \& Bokoch, 2005, Dovas \& Couchman, 2005). Interestingly, in all investigated genomes GEFs and GAPs outnumber the Rho GTPases they regulate by two- to three-fold indicating the importance and complexity of Rho activity regulation (Schmidt \& Hall, 2002, Tcherkezian \& Lamarche-Vane, 2007). GEFs as well as GAPs often contain, in addition to their catalytic domain, further domains such as a pleckstrin homology $(\mathrm{PH})$ or a citron homology $(\mathrm{CNH})$ domain. These domains could be involved in spatially and temporally regulation of the Rho proteins through integrating signals for effective crosstalk between several signalling pathways (reviewed in Cote \& Vuori, 2007, Rossman et al., 2005, Tcherkezian \& LamarcheVane, 2007). Rho proteins were shown to only exhibit their full activity when they shuttle 
between the active and inactive state (Barale et al., 2006, Fidyk et al., 2006, Vanni et al., 2005). The formation of Rho activity zones was observed in processes such as cytokinesis in a variety of organism and cell types (Bement et al., 2005), budding in yeast (Irazoqui et al., 2003, Richman et al., 2002, Wedlich-Soldner et al., 2003) and wound healing in Xenopus oocytes (Benink \& Bement, 2005). These spatially constrained zones are thought to be maintained by the balance of local Rho GTPase activation through GEFs with local Rho GTPase inactivation through GAPs. The model describing this constant flux of Rho proteins through the GTPase cycle is named the GTPase flux model (Bement et al., 2006).

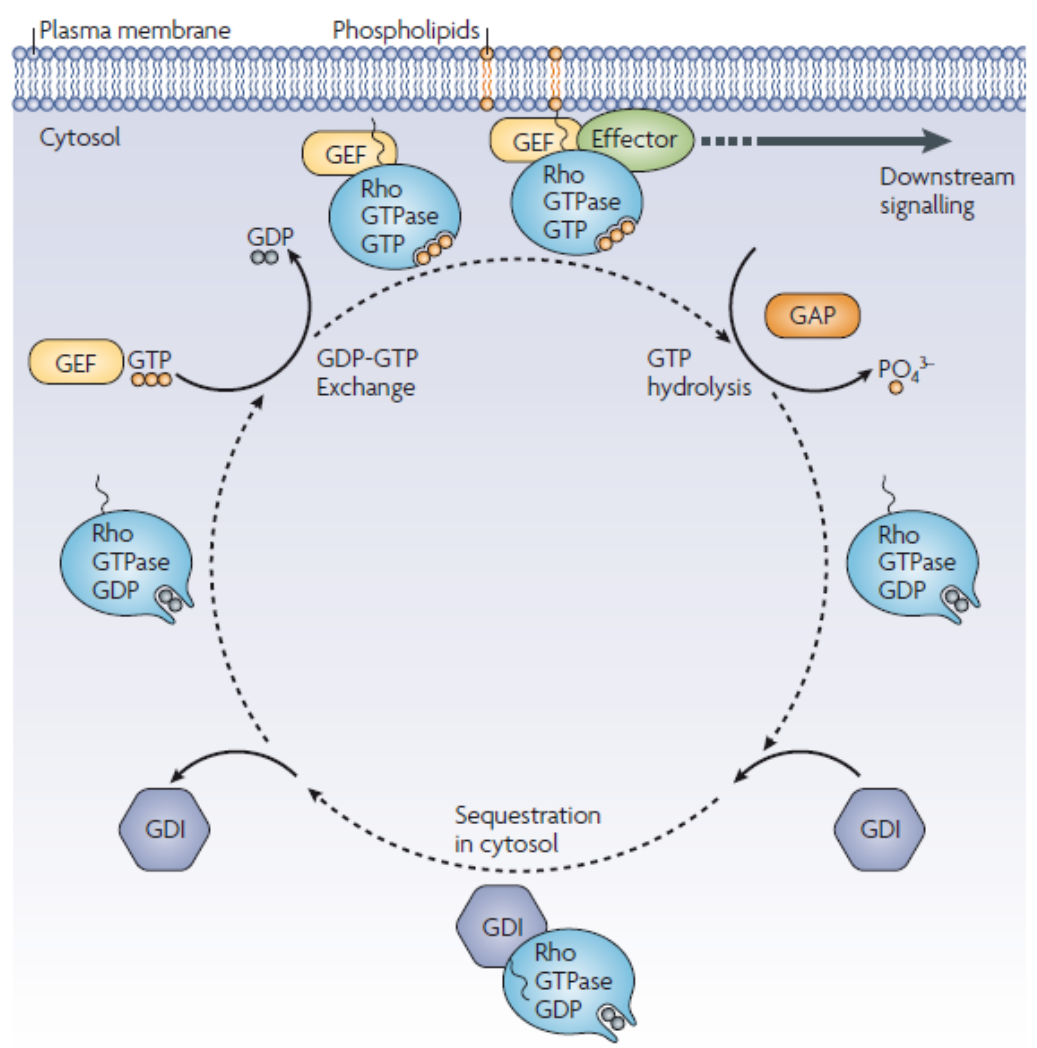

Fig. 3: Regulating Rho GTPase activity (Iden \& Collard, 2008). Rho GTPases function as molecular switches and cycle between an active GTP-bound and an inactive GDP-bound conformational state. When active, GTPbound Rho protein is predominantly associated with the membrane, where it interacts with and activates its downstream effector proteins. However, inactive GDP-bound Rho protein is present in the cytoplasm. GEF proteins are activators of Rho GTPases by stimulating the exchange of GDP for GTP. GAPs act as Rho inhibitors by stimulating the intrinsic GTPase activity of the GTPase. The release of GDP from the Rho protein is blocked by GDIs, which thereby act as inhibitors. For further details see text.

As mentioned above, Rho GTPases are key regulators of actin reorganization and regulation of cell cycle progression. Consistently, many Rho proteins are involved in cytokinesis/septation, which generally is coordinated with the cell cycle and requires the formation of a contractile actomyosin ring (see sections above). In the following Rho proteins and their function in cytokinesis/septation in different eukaryotes will be described. 


\subsubsection{Rho GTPases and cytokinesis/septation}

In animal cells RhoA, the founding member of the Rho family, is the central player in cytokinesis. Cells, in which RhoA is depleted, show no cortical contractility or furrow formation. Key RhoA effectors involved in cytokinesis are the anillin, which is a scaffold protein that links RhoA with the ring components actin and myosin during cytokinesis (Piekny \& Glotzer, 2008), formins, which promote the polymerization of actin necessary for contractile ring formation, the Rho-dependent kinase (ROCK), which regulates myosins, and the Citron kinase, which is required in some cells for the late stages of cytokinesis (reviewed in Piekny et al., 2005). Furthermore, in all eukaryotes the Rho protein Cdc42 plays a critical role in cell polarity and organization of the cytoskeleton and thus, is also involved in cytokinesis (Etienne-Manneville \& Hall, 2002). Inhibition of Rho or Cdc42 prevents the assembly of the contractile ring in many mammalian cells as well as in Xenopus embryos (Drechsel et al., 1997, Glotzer, 2001). In addition, expression of constitutively activated Rho or Cdc42 also blocks cytokinesis, suggesting that cycling between the active and inactive forms is required for function (Drechsel et al., 1997, Dutartre et al., 1996, Glotzer, 2001). In S. cerevisiae Cdc42p is essential for cytokinesis. During budding, it accumulates at the site of polarized growth and promotes, when activated through its GEF Cdc24p, the assembly of the septin ring. Septins, in turn, recruit further proteins involved in cytokinesis (see section 1.2.1). A further key regulator of cytokinesis in budding yeast is Rholp. It acts as an upstream regulator of the formin Bnilp, which in turn is necessary for actomyosin ring formation. Cells lacking functional Rho1p are deficient in actin ring formation (Tolliday et al., 2002).

The fission yeast Cdc42p and Rho1p are functional homologues of budding yeast Cdc42p and Rholp, and are essential for cell viability (Miller \& Johnson, 1994, Nakano et al., 1997). Rholp in fission yeast regulates, amongst others, septum formation and its inactivation is required for the separation of daughter cells (Nakano et al., 1997). Rgflp, Rgf2p and Rgf3p were identified as Rho1p-specific GEFs. While Rgflp and Rgf2p are involved in formation of the cell wall and the septum, Rgf3p functions in cytokinesis (Garcia et al., 2006, Mutoh et al., 2005). Furthermore, Rho5p, a functional homologue of Rholp, has also a function in controlling cell growth and division in S. pombe (Nakano et al., 2005, Rincon et al., 2006). Rho3p in budding yeast is involved in maintenance of cell polarity for bud growth by properly organizing the actin cytoskeleton during bud formation (Imai et al., 1996, Matsui \& Toh, 1992). Its counterpart in fission yeast, Rho3p, functions in polarized growth. Upon its deletion elongated and multi-septated cells are generated implying a function of Rho3p in controlling cell shape and septation (Nakano et al., 2002). While Rho3p and Rho4p in 
budding yeast have partially redundant functions and are involved in cell polarity by activating formins (Dong et al., 2003, Matsui \& Toh, 1992, Roumanie et al., 2005), Rho4p in fission yeast is a unique Rho GTPase. It is involved in septation and cell wall integrity. Rho4p localizes to the septum during mitosis and cells lacking rho4 are viable, but display cell separation defects at high temperature. This separation defect is caused by the inability to target glucanases for degradation of the primary septum to the septum (Nakano et al., 2003, Santos et al., 2003, Santos et al., 2005).

\subsubsection{Rho4 GTPase family members and septation}

As described above, fission yeast cells lacking rho4 have multiple and abnormal septa implying a function of Rho4p in septation and cell wall formation. However, rho4 deletion mutants in the hemiascomycete species $S$. cerevisiae and A. gossypii show no or only a subtle phenotype (Matsui \& Toh, 1992, Wendland et al., 2000). Phylogenetic analysis of fungal Rho proteins suggests that a new Rho-type GTPase family, whose founding member is Rho4p from the archiascomycete $S$. pombe, is involved in septation (Rasmussen \& Glass, 2005). Interestingly, CaRho4 in the hemiascomycete Candida albicans is also involved in septum construction. It localizes to septation sites in yeast and hyphal cells and upon its deletion cells show a cell separation defect (Dunkler \& Wendland, 2007). As predicted on the basis of the phylogenetic analysis, RHO4 in the filamentous euascomycete Neurospora crassa is also involved in septation as being essential (Rasmussen \& Glass, 2005). Cells lacking rho-4 are aseptate and grow slowly. Furthermore, no F-actin rings are formed in this mutant indicating a role of RHO4 in actomyosin ring formation (Rasmussen \& Glass, 2005). Localization studies revealed that RHO4 localizes to future septation sites, forms constricting rings upon septation and persists around the septal pore after septum completion. In addition, RHO4 is also observed at the plasma membrane and during conidia formation at the primary septum, of which it disappears after placement of the secondary septum (Rasmussen \& Glass, 2007).

\subsection{Aims of this work}

Cytokinesis is highly conserved throughout eukaryotes. In both yeast models great progress in understanding the principle mechanisms of cytokinesis, such as defining the future division site and formation of the contractile actomyosin ring, was made. In many filamentous fungi however, not much is known about how the nuclear cycle is coordinated with septation, how 
the future septation site is determined and how exactly the actomyosin ring and septum formation occurs. The available data of the Neurospora crassa genome revealed that many components involved in cytokinesis/septation in both yeasts and further fungi are also conserved in this fungus. At begin of this work RHO4 was the only functionally described protein showed to be required for septation in $N$. crassa. The aim of this study was to characterize further components involved in septation, such as landmark proteins, formins and septins. The work also aimed at placing the RHO4 module in the context of the septation process by identifying upstream regulators and downstream effectors of RHO4. For this purpose further characterization of the RHO4 module and identification of RHO4 activating GEFs, of which all in all seven are present in the Neurospora genome, was an additional focus. 


\section{Materials and methods}

\subsection{Media and growth conditions}

Escherichia coli strains were propagated at $37^{\circ} \mathrm{C}$ in LB medium (1\% bacto-tryptone, $0.5 \%$ yeast extract, $1 \% \mathrm{NaCl}, \mathrm{pH} 7.5$; for solid medium 1.5\% agar was added), modified from (Bertani, 1951). For selection media were used containing $100 \mu \mathrm{g} / \mathrm{ml}$ ampicillin, $50 \mu \mathrm{g} / \mathrm{ml}$ chloramphenicol or $50 \mu \mathrm{g} / \mathrm{ml}$ kanamycin (all from Sigma-Aldrich, Taufkirchen, D), respectively. For protein expression modified LB medium was used: $\mathrm{LB}^{+}(1 \% \mathrm{NaCl}, 0.8 \%$ yeast extract, $1.8 \%$ peptone, $0.4 \%$ glucose).

Saccharomyces cerevisiae was grown at $30^{\circ} \mathrm{C}$ under non-selective conditions in YEPD (2\% pepton, $1 \%$ yeast extract, $2 \%$ glucose $)$ or in SD minimal medium lacking uracil $(0.17 \%$ yeast nitrogen base (w/o amino acids, w/o ammonium sulphate), $0.5 \%$ ammonium sulphate, $10 \mathrm{mg}$ L-adenine sulphate, 10mg L-argenine, 10mg L-histidine, 15mg L-isoleucine, 50mg L-leucine, $15 \mathrm{mg}$ L-lysine, 10mg L-methionine, 25mg L-phenylalanine, 100mg L-threonine, 10mg Ltryptophane, $15 \mathrm{mg}$ L-tyrosine and $75 \mathrm{mg}$ L-valine, $2 \%$ glucose). For solid media 2\% agar was added to the respective medium.

Neurospora crassa strains were either grown in liquid or on solid (supplemented with $2 \%$ agar) Vogel's minimal medium (VMM) with 2\% (w/v) sucrose. For strains that contain the bar gene cassette, which confers glufosinate resistance, ammonium nitrate-free medium was used. Growth tests were performed in race tubes over $100 \mathrm{~h}$ to determine linear growth rates. If colonial growth of the fungus was needed, sucrose was replaced by $2 \%$ sorbose $(\mathrm{w} / \mathrm{v})$, 0.05\% glucose (w/v), and 0.05\% fructose (w/v) (Mishra \& Tatum, 1972). For crossings corn meal agar plates with 2\% (w/v) corn meal agar (Sigma, St. Louis, USA) and $0.1 \%$ glucose $(\mathrm{w} / \mathrm{v})$ were used. If needed $(150 \mu \mathrm{g} / \mathrm{ml})$ histidine or $200 \mu \mathrm{g} / \mathrm{ml}$ pantothenic acid were supplemented to the respective medium. For selection the hygromycinB (InvivoGen, San Diego, USA), nourseothricin (Werner BioAgents, Jena, D) and glufosinate-ammonium (Sigma-Aldrich, Taufkirchen, D) concentrations in the media were adjusted to $200 \mu \mathrm{g} / \mathrm{ml}, 30$ $\mu \mathrm{g} / \mathrm{ml}$ and $200 \mu \mathrm{g} / \mathrm{ml}$, respectively. General media and genetic procedures for N. crassa are also available through the Fungal Genetics Stock Center (www.fgsc.net). 


\subsection{Plasmid construction}

Primers and plasmids used in this study are listed in table 1 and table 2, respectively.

\subsubsection{MYC-tagged overexpression constructs of $r g f-3$ and $b u d-3$}

The myc-tagged full-length fusion protein construct of $r g f-3$ was generated by amplification of the NCU02131 ORF from start to stop codon (http://www.broad.mit.edu) with the primer pair DJ_2131_NotI_myc containing the $1 x$-myc-coding sequence at the very 5 ' end and DJ_2131_Stop_SpeI using the Phusion ${ }^{\circledR}$ polymerase (Finnzymes AG, Espoo, FIN). After sequencing the amplified PCR-construct was digested with NotI and SpeI and inserted into the vector pEHN1_nat (kindly provided by S. Pöggeler; Göttingen), which was digested accordingly, resulting into the plasmid pEHN1_nat_myc_rgf-3. For the generation of the rgf$3^{\Delta 865-893}$ construct, in which the putative Rho interaction helix was deleted, inverse PCR with the primer pair DJ_dhelix_2131_rev_NheI and DJ_dhelix_2131_forw_NheI and the vector pEHN1_nat_myc_rgf-3 as template was performed. The NheI restriction sites were added to the primers to create an additional restriction site for a diagnostic restriction digest. After sequencing, the construct was digested with NotI and SpeI and inserted into the vector pEHN1_nat, which was digested accordingly, resulting into the plasmid pEHN1_nat_myc_sHelix_rgf-3. The full-length myc-tagged bud-3 construct was generated by amplification of the NCU06579 ORF from start to stop codon (http://www.broad.mit.edu) with the primers DJ_6579_SpeI_myc containing the $1 x$-myc-coding sequence at the very 5' end and DJ_6579_Stop_Kpn2I using the Phusion ${ }^{\circledR}$ polymerase (Finnzymes AG, Espoo, FIN). To obtain the plasmid pEHN1_nat_myc_bud-3 the sequenced PCR-construct was introduced via SpeI and Kpn2I into the vector pEHN1nat.

\subsubsection{GFP constructs for localization studies}

GFP-tagged fusion proteins were generated by amplification of the ORFs from start to stop codons as annotated by the $N$. crassa database (http://www.broad.mit.edu), for cloning purposes restriction sites were added to the ends of the primers. For $r g f-3$ the primer pair DJ_2131_rev_PacI and DJ_SpeI_2131_ATG, for bud-3 DJ_SpeI_6579_ATG and DJ_6579_rev_XmaI, for bud-4 (NCU00152) DJ_BUD4_SpeI_ATG and DJ_BUD4_rev_PacI, for bni-1 (NCU01431) DJ_BNI1_SpeI_ATG_and DJ_BNI1_rev_PacI, for $c d c-12$ (NCU03795) DJ_CDC12_SpeI_ATG and DJ_CDC12_rev_PacI and for cla-4 (NCU00406) 
the primer pair DJ_CLA4_XbaI_ATG and DJ_CLA4_rev_PacI were used. PCR was performed with the Phusion ${ }^{\circledR}$ polymerase (Finnzymes AG, Espoo, FIN) following the manufacturer's protocol or with the $P f u$-polymerase (Fermentas GmbH, St. Leon-Rot, D) using genomic wild type DNA as template. All PCR-amplified constructs were verified by sequencing. The amplified fragments were digested with PacI/SpeI ( $r g f-3$, bud-4, cdc-12 and bni-1), PacI/XbaI (cla-4) and XmaI/SpeI (bud-3) and inserted into the vector pMF272 (Freitag et al., 2004), which was digested accordingly, leading to the plasmids pMF272_rgf-3_sgfp, pMF272_bud-3_sgfp, pMF272_bud-4_sgfp, pMF272_cdc-12_sgfp, pMF272_cla-4_sgfp and pMF272_bni-1_sgfp. The GFP-tagged truncated versions of $r g f-3$ were generated by PCR using the primer pairs DJ_SpeI_GEF_PH_2131_f and DJ_PacI_PH_GEF_2131_r for RGF3_GEF_PH, DJ_SpeI_PH_CNH_2131_f and DJ_PacI_CNH_PH_2131_r for RGF3_PH_CNH and DJ_SpeI_ATG_CNH_2131_f and DJ_PacI_CNH_2131_r for RGF3_CNH and inserted in PacI/SpeI-digested vector pMF272 to obtain the plasmids pMF272_RGF3_GEF_PH, pMF272_RGF3_PH_CNH and pMF272_RGF3_CNH. Alternatively, a nourseothricin resistence (nat) cassette was amplified by PCR using the primers DJ_5'ApaI_natR_f and DJ_3'ApaI_natR_r (for pMF272_bud-3-sgfp_nat) or DJ_5'NotI_natR_f and DJ_3'NotI_natR_r (for pMF272_bni-1-sgfp_nat) and pEHN1nat as template. Subsequently, the cassette was introduced via ApaI into pMF272_bud-3_sgfp and via NotI into pMF272_bni-1_sgfp resulting in the final plasmids pMF272_bud-3-sgfp_nat and pMF272_bni-1-sgfp_nat.

\subsubsection{Maltose binding protein (MBP) constructs for in vitro GEF assays}

MBP-tagged N. crassa GEF constructs containing the GEF domain and $\mathrm{PH}$ and $\mathrm{CNH}$ domain if available were cloned by reverse transcription (RT)-PCR using the RevertAid M-MuLV Reverse Transcriptase (Fermentas GmbH, St. Leon-Rot, D) and the primers CoS_06579_3 and CoS_06579_4 for bud-3 and NV_2131_1 and NV_2131_2 for rgf-3 with total RNA prepared by TRIzol extraction (Invitrogen $\mathrm{GmbH}$, Karlsruhe, D) from vegetative hyphae as template. For cloning of the two Aspergillus nidulans constructs Anrho-4 (AN2687.3) containing the whole coding sequence and Anbud-3 (AN0113.3) with the GEF domain enclosed the primers DJ_AN2687rho4_NcoI_f and DJ_HindIII_AN2687rho4_r for rho-4 and DJ_AN0113_NcoI_f and DJ_AN0113_NotI_TGA_rev for bud-3 were used for PCR with a cDNA pool as template prepared from vegetative hyphae (kindly provided by M. Christmann and R. Harting; Institute for Microbiology and Genetics, University of Göttingen, D). The amplified constructs were subcloned into pJet1.2/blunt vector (Fermentas GmbH, St. Leon- 
Rot, D) and sequenced. In the process of generating cDNA for $r g f-3$ two different splice variants were found that differed in the sequence of the PH domain (NCBI accession no. GQ401155 and GQ401156). The MBP fusion constructs were digested either with SalI and NotI (bud-3), EcoRV and HindIII (rgf-3), NcoI and NotI (anbud-3) or NcoI and HindIII (anrho-4) and inserted into a modified pMalc2x vector (Vogt \& Seiler, 2008), which was digested accordingly to generate the plasmids pMal_RGF3_short, pMal_RGF3_long, pMal_BUD3, pMal_AnBud3 and pMal_AnRho4. The plasmids for the expression of the six MBP- or GST-tagged N. crassa Rho proteins are described in (Vogt, 2008).

\subsubsection{Deletion cassettes for the double knockout $\Delta r g f-3 ; \Delta b u d-3$}

The deletion cassette for $\Delta r g f-3$ containing ca. $1 \mathrm{~kb}$ of $5^{\prime}$ rgf-3 flanking sequence, the marker $h p h$ leading to a hygromycin resistance and ca. $1 \mathrm{~kb}$ of 3' rgf-3 flanking sequence was amplified by PCR from genomic DNA of the $\Delta r g f-3$ strain using the primer pair DJ_5UTR_2131_f and DJ_3UTR_2131_r and was subcloned into pJet1.2/blunt vector (Fermentas GmbH, St. Leon-Rot, D). The deletion construct for the knockout of $\Delta b u d-3$ containing ca. $1 \mathrm{~kb}$ of 5 ' bud-3 flanking sequence, the nourseothricin resistance cassette and ca. $1 \mathrm{~kb}$ of 3' bud-3 flanking sequence was created utilizing homologous recombination in yeast (Colot et al., 2006). For amplification of the knockout cassette, the Phusion polymerase (Finnzymes AG, Espoo, FIN) was used following the manufacturer's instructions. The 5'UTR and 3' UTR regions of the bud-3 gene were amplified from genomic DNA of the $N$. crassa wild type strain with following primer pairs: DJ_pRS426_5UTR_6579_f and DJ_5UTR_pnat_6579_r and DJ_pnat_3UTR_6579_f and DJ_3UTR_6579_pRS426_r. Specific 29-bp overhangs were added to the primers that were homologous to the yeast plasmid pRS426 (Christianson et al., 1992) and the nourseothricin resistance cassette, respectively. The nourseothricin resistance cassette was generated from pEHN1nat vector using the primers DJ_5'ApaI_natR_f and DJ_3'ApaI_natR_r. The three obtained PCR fragments were co-transformed together with the EcoRI-XhoI linearized plasmid pRS426 by electroporation according to (Becker \& Lundblad, 2001) at $1.5 \mathrm{kV}$ into the yeast strain PJ694A (James et al., 1996), where homologous recombination took place. Yeast DNA was isolated from PJ69-4A by Smash and Grab (Rose et al., 1990) and transformed into E. coli. The recombinant plasmid pRS426_5'UTR_nat_3'UTR_bud-3 containing the bud-3 deletion cassette was isolated and subsequently sequenced. 


\subsubsection{DBF2 $2^{\mathrm{T} 671 \mathrm{E}}$ plasmid}

The vector pEHN1_nat_myc_his_dbf- $2^{\mathrm{T} 671 \mathrm{E}}$ was obtained by PCR-based site-directed mutagenesis with the primer pair DJ_DBF2_T2E_f and DJ_DBF2_T2E_r and pEHN1_nat_myc_his_dbf-2 as template. The PCR was performed with the Phusion polymerase (Finnzymes AG, Espoo, FIN) as described in section 2.4.2. The obtained PCR products were digested with $D p n I$ to separate the new mutated non-methylated target plasmid from the non-mutated methylated input plasmid and subsequently transformed into E. coli. The mutation was verified by sequencing and the mutated $d b f-2$ construct was introduced into the pEHN1nat vector via AscI and BamHI.

Table 1: Primers used in this study.

\begin{tabular}{ll}
\hline Primer name & Sequence (restriction sites are underlined) \\
\hline CoS_06579_3 & ATGTCGACCCCTTCGCGGCCCTCTTCAT \\
CoS_06579_4 & TGCGGCCGCTTAGGCCTTGGCGATTTCTTCAC \\
NV_2131_1 & CGATATCGGCATTCCAGTAAGGTGCTAC \\
NV_2131_2 & CAAGCTTCTAAGCCTTCTCCGCATGCC \\
DJ_2131_rev_PacI & CCGTTAATTAAAGCCTTCTCCGCATGCCCATC \\
DJ_SpeI_2131_ATG & GGCACTAGTATGTCGTTTCGCGGCGACGATTC \\
DJ_SpeI_6579_ATG & GCACTAGTATGGTTCGTGTCACGGAGGAAC \\
DJ_6579_rev_XmaI & CCGCCCGGGTGACCCGTCAACCCCATCAAG \\
DJ_5'ApaI_natR_f & GGGCCCGAATTCAACTGATATTGAAGGAGC \\
DJ_3'ApaI_natR_r & GGGCCCCTCAGGGGCAGGGCATGCTC \\
DJ_5'NotI_natR_f & GCGGCCGCGAATTCAACTGATATTGAAGGAGC \\
DJ_3'NotI_natR_r & GCGGCCGCCTCAGGGGCAGGGCATGCTC \\
DJ_BUD4_SpeI-ATG & GGCACTAGTATGGCGTCGGAGGAACCGG \\
DJ_BUD4_rev_PacI & CCGTTAATTAATACAAGACTGCTCTTCGTTC \\
DJ_BNI1_SpeI_ATG & GGCACTAGTATGTCCTCCCACGACAAGAATG \\
DJ_BNI1_rev_PacI & CCGTTAATTAATCCTGTTGTTTCTTTACTTTCTTC \\
DJ_CDC12_SpeI_ATG & GGCACTAGTATGGCCCCTGCCACTACCG \\
DJ_CDC12_rev_PacI & CCGTTAATTAAGCGGCGACCGTGGCTGCG \\
DJ_CLA4_XbaI_ATG & GGCTCTAGAATGGCGCAAAATGGGTATGC \\
DJ_CLA4_rev_PacI & CCGTTAATTAATTTCGCCGACCTCTTGAAGG \\
DJ_AN2687RHO4_NcoI_f & CCGCCATGGGCTCTGGCTCAATGTACGATGACC \\
DJ_HindIIIAAN2687RHO4_r & CCGAAGCTTTCACAGAATCTTGCAGCTGCG \\
DJ_AN0113_NcoI_f & CCGCCATGGGGCAACCAACCAATGTCAGTCG \\
DJ_AN0113_Not__TGA_rev & CCGGCGGCCGCTCACGACCATTTGCCACTCTCTC \\
DJ_PacI_PH_GEF_2131_r & GCCTTAATTAACTCGGCGTTCTGTGCATGTAG \\
DJ_SpeI_GEF_PH_2131_f & GGCACTAGTTATGTCGTTTCGCGGCGACG \\
DJ_SpeI_PH_CNH_2131_f & GGCACTAGTATGGGTTTCCAATCAGTCCTCAATC \\
DJ_PacI_CNH_PH_2131_r & GGCTTAATTAAAGCCTTCTCCGCATGCCC \\
DJ_SpeI_ATG_CNH_2131_f & GGCACTAGTATGGTATGCAGAGCTCAGGTAAAC \\
DJ_PacI_CNH_2131_r & GGCTTAATTAAAGCCTTCTCCGCATGCCC \\
DJ_2131_Not_myc & CTCGCGGCCGCGGGTGAGCAGAAGCTGATCTCCGAGGAA \\
DJ_2131_Stop_SpeI & GACCTCAACTCGTTTCGCGGCGACGATTCACG \\
DJ_dheli_2131_r_NheI & CGCACTAGTCTAAGCCTTCTCCGCATGCC \\
DJ_dhelix_2131__N_NheI & CCGGCTAGCCTCAATCTCCTTTTTGCTCTTCTTTTC \\
\hline
\end{tabular}


Table 1 (continued): Primers used in this study.
Primer name
DJ_6579_SpeI_myc
DJ_6579_Stop_Kpn2I
DJ_DBF 2-T2E $\mathrm{f}$
DJ DBF2 T2E $r$

Sequence (restriction sites are underlined)

AGAACTAGTGGGTGAGCAGAAGCTGATCTCCGAGGAAGA CCTCAACGTTCGTGTCACGGAGGAACTGG

CAACTCCGGATCATGACCCGTCAACCCC

GTTTGTCGGATTCgagTTCCGTCATCGCAAG

CTTGCGATGACGGAActcGAATCCGACAAAC

Table 2: Plasmids used in this study.

\begin{tabular}{|c|c|c|}
\hline Plasmid name & Description & Source \\
\hline pMF272 & $\begin{array}{l}\text { Cloning vector for C-terminal GFP } \\
\text { fusions under control of the ccg-1 } \\
\text { promoter }\end{array}$ & Freitag et al., 2004 \\
\hline pEHN1nat & $\begin{array}{l}\text { Cloning vector with the gpd } \\
\text { promoter and the nat-resistance } \\
\text { cassette }\end{array}$ & $\begin{array}{l}\text { S. Pöggeler, } \\
\text { Göttingen, D }\end{array}$ \\
\hline pJet1.2/blunt & $\begin{array}{l}\text { Cloning vector of CloneJETTM } \\
\text { PCR Cloning Kit for subcloning of } \\
\text { PCR fragments }\end{array}$ & $\begin{array}{l}\text { MBI Fermentas, St. } \\
\text { Leon-Rot, D }\end{array}$ \\
\hline pNV72 & $\begin{array}{l}\text { pMal-c2x; changed multiple } \\
\text { cloning site, expression plasmid for } \\
\text { MalE fusions }\end{array}$ & N. Vogt, 2008 \\
\hline pRS426 & $\begin{array}{l}\text { Yeast/E.coli vector for homologous } \\
\text { recombination in yeast; contains } \\
\text { URA3 gene sequence }\end{array}$ & Christianson et al., 1992 \\
\hline pMal_BUD3 & $p N V 72 ;$ bud-3 $3_{a a 230-637} c D N A$ & $\begin{array}{l}\text { C. Richthammer, } \\
\text { Göttingen, D }\end{array}$ \\
\hline pMal_RGF3_short & $p N V 72 ; r g f-3_{\text {aa811-1672 }} c D N A$ & This study \\
\hline pMal_RGF3_long & $p N V 72 ; r g f-3_{a a 811-1688} c D N A$ & This study \\
\hline pMal_AnBud3 & pNV72; anbud-3 aa230-647 cDNA & This study \\
\hline pMal_AnRho4 & pNV72;anrho-4 cDNA & This study \\
\hline pMal_RHO1 & pNV72;rho-1 cDNA & N. Vogt, 2008 \\
\hline pMal_RHO2 & pNV72;rho-2 cDNA & N. Vogt, 2008 \\
\hline pMal_RHO3 & pNV72;rho-3 cDNA & N. Vogt, 2008 \\
\hline pMal_RHO4 & pNV72;rho-4 cDNA & N. Vogt, 2008 \\
\hline pMal_RAC & $p N V 72 ; r a c$ cDNA & N. Vogt, 2008 \\
\hline pMal_CDC42 & $p N V 72 ; c d c-42$ cDNA & N. Vogt, 2008 \\
\hline pETM-30 & $\begin{array}{l}\text { expression plasmid for GST } \\
\text { fusions }\end{array}$ & $\begin{array}{l}\text { protein expression } \\
\text { facility, Heidelberg, D }\end{array}$ \\
\hline pNV28 & pETM-30;rho-1 cDNA & Vogt \& Seiler, 2008 \\
\hline pNV29 & pETM-30;rho-2 cDNA & Vogt \& Seiler, 2008 \\
\hline pNV30 & pETM-30;rho-3 cDNA & Vogt \& Seiler, 2008 \\
\hline pNV31 & pETM-30;rho-4 cDNA & Vogt \& Seiler, 2008 \\
\hline pNV32 & pETM-30;rac cDNA & Vogt \& Seiler, 2008 \\
\hline pNV33 & pETM-30;cdc-42 cDNA & Vogt \& Seiler, 2008 \\
\hline pMF272_rgf-3_sgfp & pMF272;rgf-3 coding sequence & This study \\
\hline pMF272_bud-3_sgfp & pMF272;bud-3 coding sequence & This study \\
\hline pMF272_bud-4_sgfp & pMF272;bud-4 coding sequence & This study \\
\hline pMF272_bni-1_sgfp & pMF272;bni-1 coding sequence & This study \\
\hline pMF272_bud-3-sgfp_nat & pMF272_bud-3_sgfp; $P_{\text {gpdA }}::$ nat $^{R}$ & This study \\
\hline pMF272_bni-1-sgfp_nat & pMF272_bni-1_sgfp; $\mathrm{P}_{\mathrm{gpd} A}: \mathrm{nat}^{\mathrm{R}}$ & This study \\
\hline pMF272_cdc-12_sgfp & pMF272;cdc-12 coding sequence & This study \\
\hline pMF272_cla-4_sgfp & pMF272;cla-4 coding sequence & This study \\
\hline
\end{tabular}


Table 2 (continued): Plasmids used in this study.

\begin{tabular}{|c|c|c|}
\hline Plasmid name & Description & Source \\
\hline pMF272_rgf-3_GEF_PH & $\begin{array}{l}\text { pMF272;rgf-3 } 3^{(1-1270)} \text { coding } \\
\text { sequence }\end{array}$ & This study \\
\hline pMF272_rgf-3_PH_CNH & $\begin{array}{l}\text { pMF272;rgf-3 } 3^{(1077-1688)} \text { coding } \\
\text { sequence }\end{array}$ & This study \\
\hline pMF272_rgf-3_CNH & $\begin{array}{l}\text { pMF272;rgf-3 } 3^{(1323-1688)} \text { coding } \\
\text { sequence }\end{array}$ & This study \\
\hline pEHN1_nat_myc_rgf-3 & $\begin{array}{l}\text { pEHN1_nat; } m y c-r g f-3 \text { coding } \\
\text { sequence }\end{array}$ & This study \\
\hline pEHN1_nat_myc_bud-3 & $\begin{array}{l}\text { pEHN1_nat; } m y c-b u d-3 \text { coding } \\
\text { sequence }\end{array}$ & This study \\
\hline pEHN1_nat_myc_AHelix_rgf-3 & $\begin{array}{l}\text { pEHN1_nat; } m y c-r g f-3^{4865-893} \\
\text { coding sequence }\end{array}$ & This study \\
\hline pEHN1_nat_myc_his_dbf-2 & $\begin{array}{l}\text { pEHN1_nat; } m y c^{5}-h i s^{6}-d b f-2 \text { coding } \\
\text { sequence }\end{array}$ & S. März, Göttingen, D \\
\hline pEHN1_nat_myc_his_dbf-2 ${ }^{\mathrm{T} 671 \mathrm{E}}$ & $\begin{array}{l}\text { pEHN1_nat; } m y c^{5}-h i s^{6}-d b f-2^{T 671 E} \\
\text { coding sequence }\end{array}$ & This study \\
\hline pRS426_5'UTR_nat_3'UTR_bud-3 & $\begin{array}{l}\text { Yeast vector pRS } 426 \text { containing } \\
\text { the bud-3 deletion cassette ( } 5 \\
\text { flanking sequences of bud-3, the } \\
\text { nat }{ }^{\mathrm{R}} \text { cassette and 3' flanking } \\
\text { sequences of } b u d-3 \text { ) for generation } \\
\text { of the double knockout } \Delta r g f- \\
3 ; \Delta b u d-3\end{array}$ & This study \\
\hline pJet_5'UTR_hph_3'UTR_rgf-3 & $\begin{array}{l}\text { Vector containing the } r g f-3 \\
\text { deletion cassette (5' flanking } \\
\text { sequences of } r g f-3 \text {, the hygromycin } \\
\text { resistance cassette }(h p h) \text { and } 3 \text {, } \\
\text { flanking sequences of } r g f-3 \text { ) for } \\
\text { generation of the double knockout } \\
\Delta r g f-3 ; \Delta b u d-3\end{array}$ & This study \\
\hline
\end{tabular}

\subsection{Strains}

\subsubsection{E. coli}

For general cloning procedures the Escherichia coli strain DH5 $\alpha \mathrm{F}^{-}, \Phi 80 \mathrm{~d} \Delta\left(\right.$ lacZ)M15 ${ }^{-1}$, $\Delta(\operatorname{lacZYA}-\arg F) \mathrm{U} 169, \operatorname{rec} A 1$, endA1, hsdR17 $\left(\mathrm{r}_{\mathrm{K}}^{-}, \mathrm{m}_{\mathrm{K}}{ }^{+}\right), \operatorname{supE44,} \lambda^{-}$, thil, gyrA96, relA1] (Woodcock et al., 1989) was used. Protein expression was done with E. coli Rosetta2 (DE3) cells (Merck KGaA, Darmstadt, Germany).

\subsubsection{S. cerevisiae}

The yeast strain PJ69-4A (MAT $\alpha$; trpl-901; leu2-3112; ura3-52, his3-200; ga14A; ga18O $\Delta$; LYS2::GAL1-HIS3, GAL2-ADE2; met2::GAL7-lacZ; James et al., 1996) was used for generation of the deletion construct for the knockout of the bud3-gene in $N$. crassa through homologous recombination (Orr-Weaver \& Szostak, 1983). 


\subsubsection{N. crassa}

Neurospora crassa strains used in this study are summarized in table 1.

All single deletion strains used in this study were generated by the $N$. crassa genome project (Dunlap et al., 2007; http://www.dartmouth.edu/ neurosporagenome). Their full-length open reading frames were replaced by a hygromycin resistance cassette and the strains were verified by Southern analysis. $\Delta r g f-3, \Delta b u d-3, \Delta b u d-4$ and $\Delta r h o-4$ were deposited at the Fungal Genetics Stock Center as heterokaryons carrying two types of nuclei: one harbouring the deletion (marked by the hygromycin resistance) and a second wild type nucleus that shelters the deletion defect. Homokaryotic deletion strains of $\Delta r g f-3, \Delta b u d-3, \Delta b u d-4$ and $\Delta r h o-4$ were obtained by back-crosses of the respective heterokaryotic deletion strain with wild type and selecting for progeny carrying the deletion nucleus.

To express dominant-active RHO4 into $\Delta r g f-3$ or $\Delta b u d-3$ the two strains rho-4(Q154L) and rho-4(G103V) containing HA-tagged dominant-active versions of RHO4 were crossed with the respective heterokaryotic deletion strain. After selecting for progeny carrying the deletion nucleus, the expression of the respective daRHO4 variant in the respective deletion strain was verified by Western blotting using Anti-HA antibodies. For expression of the GFP fusion proteins RGF3-GFP, BUD3-GFP, BUD4-GFP, BNI1-GFP, CDC12-GFP and CLA4-GFP and the truncated mutants of RGF-3 RGF3_GEF_PH, RGF3_PH_CNH and RGF3_CNH in wildtype background the respective plasmid enlisted in table 3 was targeted to the his-3 locus via homologous recombination by transformation into his-3- $3^{-}$spheroplasts (FGSC \#6103) as described by (Vollmer \& Yanofsky, 1986), thus complementing the histidine auxotrophy. GFP-positive strains of the different fusion proteins were identified by fluorescence microscopy and Western blot experiments. The complementation strains rgf-3-sgfp, bud-4$s g f p$ and $b n i-1-s g f p$ were obtained by crossing the respective GFP fusion protein-expressing strain with the respective heterokaryotic deletion strain. Progeny growing on selective medium were further tested for expression of the respective GFP fusion protein as described above. Likewise, the strains rgf-3-sgfp; $\Delta b u d-3, r g f-3-s g f p ; \Delta b u d-4$, sgfp-rho-4; $\Delta r g f-3$, sgfprho-4; $\Delta b u d-3$, sgfp-rho-4; $\Delta b u d-4$, bud-4-sgfp; $\Delta r g f-3$, bud-4-sgfp; $\Delta b u d-3, \quad r g f-3(G E F-P H)$ sgfp; $\Delta r g f-3$ and $r g f-3(P H-C N H)-s g f p ; \Delta r g f-3$ were generated via crosses of the respective strain into the desired deletion background. For bud-3-sgfp, bud-3-sgfp; $\Delta r g f-3$ and bni-1$s g f p ; \Delta r g f-3$ another strategy was used since crosses were not successful: Forced heterokarya with wild-type characteristics between the aseptate (and thus aconidiate) homokaryotic deletion strains $\Delta b u d-3$ and $\Delta r g f-3$ and FEN71-18 were generated to facilitate the genetic manipulation via DNA transformation. For this purpose, conidia of the FEN71-18 strain were 
added to the respective deletion strain grown on solid VMM and fusion of both strains was monitored by growth of the heterokaryon with wild type characteristics on medium supplemented with benomyl and hygromycin. Spheroplasts of the resulting heterokaryotic strains $\Delta b u d-3+F E N 71-18$ and $\Delta r g f-3+F E N 71-18$ were prepared and the plasmids pMF272_bud-3-sgfp_nat and pMF272_bni-1-sgfp_nat were introduced into the respective strains. Afterwards, transformants growing on hygromycin- and nourseothricin (nat)containing medium were tested for GFP fusion protein-expression by microscopy and single spores of these identified strains were isolated and then tested for the presence/absence of the sheltering nucleus by growth on $5 \mu \mathrm{g} / \mathrm{ml}$ benomyl and $200 \mu \mathrm{g} / \mathrm{ml}$ pantothenic acid. Positive isolates of bud-3-sgfp, bud-3-sgfp; $\operatorname{rgf-3,}$ bni-1-sgfp; $\operatorname{rgf-3}$ and bni-1-sgfp; $\Delta b u d-3$ were further characterized. Likewise, the complemented strain myc-rgf-3; $\operatorname{rgf} f-3$ was obtained. The plasmid pEHN1_nat_myc_rgf-3 was introduced into the $\Delta r g f-3+$ FEN71-18 strain and single spores were isolated that were unable to grow on benomyl-containing medium, but were nourseothricin- and hygromycin- resistant. The myc-rgf-3; $\operatorname{rgf}-3$ strain showed wild type growth and the expression of MYC-RGF3 was verified in Western blot experiments. To generate a hyper-active mutant of DBF2 the vector pEHN1_nat_myc_his_dbf-2 ${ }^{\mathrm{T} 671 \mathrm{E}}$ was transformed into wild type spheroplasts. Nourseothricin-resistant transformants expressing the mutated $\mathrm{MYC}^{5}$-HIS ${ }^{6}$-tagged $\mathrm{DBF} 2^{\mathrm{T} 671 \mathrm{E}}$ were verified by Western blot analysis using Anti-cmyc antibodies. For generation of the double knockout mutant $\Delta r g f-3 ; \Delta b u d-3$ the plasmid pJet_5'UTR_hph_3'UTR_rgf-3 containing the rgf-3 deletion cassette, which consists of ca. 1 $\mathrm{kb}$ of $5^{\prime}$ sequence flanking the $r g f-3 \mathrm{ORF}$, the selectable marker $h p h$, which confers to hygromycin resistance driven by the $\operatorname{trp} C$ promoter, and ca. $1 \mathrm{~kb}$ of 3' flanking sequence of the rgf-3 ORF, and the plasmid pRS426_5'UTR_nat_3'UTR_bud-3 containing the bud-3 deletion cassette, which consists of ca.1 kb of 5' sequence flanking the bud-3 ORF, the selectable nourseothricin marker cassette, driven by the Pgpd promoter, and ca $1 \mathrm{~kb}$ of 3 , flanking sequence of the bud-3 ORF, were linearized with NcoI and HindIII, respectively, and subsequently introduced into $\Delta m u s-52: \because$ bar $^{R}$; his-3 mat A (\#FGSC 9720, Colot et al., 2006) spheroplasts. Primary transformants growing on hygromycin- and nourseothricin-containing medium were screened by Southern hybridisation analyses (Sambrook et al., 1989). 
Table 3. N. crassa strains used in this study

\begin{tabular}{|c|c|c|}
\hline Strain & Genotype & Source \\
\hline wild type & 74-OR23-1A & FGSC \#987 \\
\hline wild type & ORS-SL6 a & FGSC \#4200 \\
\hline his-3- & his-3 & FGSC \#6103 \\
\hline$\Delta m u s-52 ; h i s-3$ & $m u s-52 \Delta::$ bar $^{R} ;$ his-3 A & FGSC\#9720 \\
\hline FEN71-18 & $p a n-2 ; b m l^{R}$ & Nargang et al., 1995 \\
\hline$\Delta r g f-3$ (heterokaryon) & 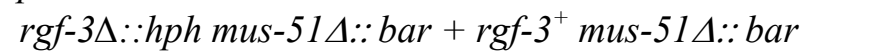 & FGSC \#11493 \\
\hline srgf-3 (homokaryon) & 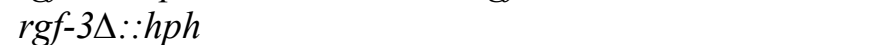 & FGSC \#11493 x FGSC \#987 \\
\hline$\Delta r g f-3+F E N 71-18$ & $\operatorname{rgf}-3 \Delta:: h p h+p a n-2 b m l^{R}$ & This study \\
\hline$r g f-3-s g f p$ & his- $3^{+}:: P c c g-1-r g f-3-s g f p$ & This study \\
\hline rgf-3-sgfp (complemented) & 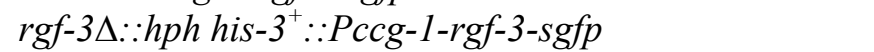 & This study \\
\hline$\Delta b u d-3$ (heterokaryon) & 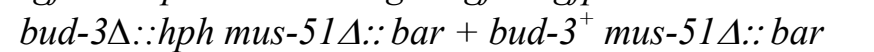 & FGSC \#19734 \\
\hline$\Delta$ bud-3 (homokaryon) & 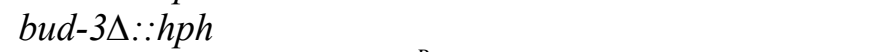 & FGSC \#19734 x FGSC \#987 \\
\hline$\Delta b u d-3+F E N 71-18$ & 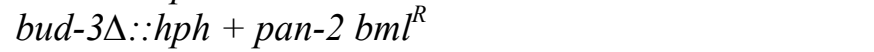 & This study \\
\hline bud-3-sgfp & his-3 ${ }^{+}:: P c c g-1-b u d-3-s g f p$ & This study \\
\hline bud-3-sgfp (complemented) & 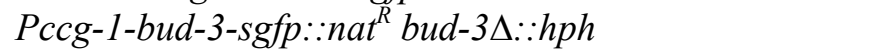 & This study \\
\hline$\Delta$ bud-4 (heterokaryon) & 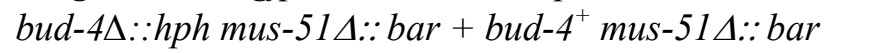 & FGSC \#13505 \\
\hline$\Delta$ bud-4 (homokaryon) & 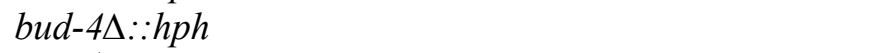 & FGSC \#13505 x FGSC \#987 \\
\hline$b u d-4-s g f p$ & $h i s-3^{+}:: P c c g-1-b u d-4-s g f p$ & This study \\
\hline bud-4-sgfp (complemented) & 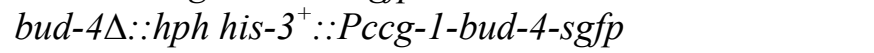 & This study \\
\hline$\Delta$ bni-1 (heterokaryon) & 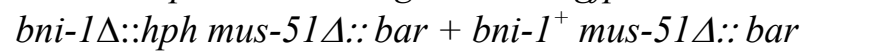 & FGSC \#11490 \\
\hline$b n i-1-s g f p$ & $h i s-3^{+}: \because P c c g-1-b n i-1-s g f p$ & This study \\
\hline bni-1-sgfp(complemented) & bni-1A::hph his- $3^{+}: \because$ Pccg-1-bni-1-sgfp & This study \\
\hline$c d c-12-s g f p$ & $h i s-3^{+}: \because P c c g-1-c d c-12-s g f p$ & This study \\
\hline cla-4-sgfp & $h i s-3^{+}: \because P c c g-1-c l a-4-s g f p$ & This study \\
\hline rho-4 (heterokaryon) & 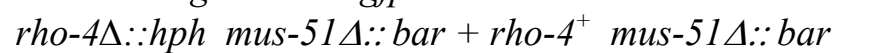 & FGSC \#11726 \\
\hline rho-4 (homokaryon) & rho- $4 \Delta:: h p h$ & FGSC \#11726 x FGSC \#987 \\
\hline CR5-10 & ad-3A his-3 rho-4 $4^{R I P 154}+a d-3 B$ cyh-a am ${ }^{l}$ & Rasmussen \& Glass, 2005 \\
\hline sgfp-rho-4; $\Delta s a d-1$ & Sad-1::hph his- $3^{+}: \because P c c g 1-r h o-4-s g f p$ & FGSC \#10195 \\
\hline rho-4(RIP) + FEN71-18 & ad-3A his-3 rho-4 $4^{R I P 154}+$ pan-2 bml ${ }^{R}$ & This study \\
\hline rho-4(RIP);rgf-3-sgfp & his- $3^{+}: \because P c c g-1-r g f-3-s g f p$ rho- $4^{R I P I 54}$ & This study \\
\hline rho-4(RIP); bud-3-sgfp & his- $3^{+}:: P c c g-1-b u d-3-s g f p$ rho- $4^{R I P 154}$ & This study \\
\hline$r g f-3-s g f p ; \Delta b u d-3$ & 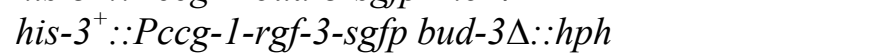 & This study \\
\hline$r g f-3-s g f p ; \Delta b u d-4$ & $h i s-3^{+}: \because P c c g-1-r g f-3-s g f p$ bud- $4 \Delta:: h p h$ & This study \\
\hline bud-3-sgfp; $\Delta r g f-3$ & Pccg-1-bud-3-sgfp::nat ${ }^{R}$ rgf-3A::hph & This study \\
\hline bud-3-sgfp; $\Delta$ bud-4 & his- $3^{+}: \because P c c g-1-b u d-3-\operatorname{sgfp}$ bud- $4 \Delta::$ hph & This study \\
\hline bud-4-sgfp; $\Delta r g f-3$ & $h i s-3^{+}: \because P c c g 1-b u d-4-s g f p$ rgf- $3 \Delta:: h p h$ & This study \\
\hline bud-4-sgfp; $\Delta$ bud-3 & his- $3^{+}: \because P c c g 1-b u d-4-s g f p$ bud- $3 \Delta:: h p h$ & This study \\
\hline rho-4(Q154L); $\Delta r g f-3$ & his- $3^{+}:: P g p d-H A-Q 154 L-r h o-4$ rho-4 ${ }^{R I P 154}$ rgf-3A $:: h p h$ & FGSC \#10188 x FGSC \#11493 \\
\hline rho-4(Q154L); $\Delta$ bud -3 & his- $3^{+}::$Pgpd-HA-Q154L-rho-4 rho-4 ${ }^{\text {RIPI } 154}$ bud-3A.: hph & FGSC \#10188 x FGSC \#19734 \\
\hline rho-4(G103V); $\Delta b u d-3$ & his- $3^{+}::$Pgpd-HA-G103V-rho-4 rho-4 ${ }^{\text {RIPI54 }}$ bud-3A ::hph & FGSC \#10184 x FGSC \#19734 \\
\hline sgfp-rho-4; $\Delta r g f-3$ & 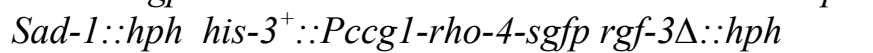 & FGSC \#10195 x $\Delta r g f-3$ \\
\hline sgfp-rho-4; $\Delta$ bud-3 & 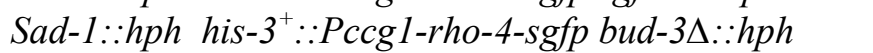 & FGSC \#10195 x $\Delta b u d-3$ \\
\hline sgfp-rho-4; $\Delta$ bud-4 & Sad-1::hph his- $3^{+}::$Pccg1-rho-4-sgfp bud-4A::hph & FGSC \#10195 x $\Delta b u d-4$ \\
\hline bni-1-sgfp; $\Delta r g f-3$ & Pccg-1-bni-1-sgfp::nat ${ }^{R}$ rgf-3A:: hph & This study \\
\hline bni-1-sgfp; $\Delta b u d-3$ & Pccg-1-bni-1-sgfp::nat ${ }^{R}$ bud-3A::hph & This study \\
\hline$r g f-3(G E F-P H)-s g f p$ & his- $3^{+}: \because P c c g-1-r g f-3(1-1270)-s g f p$ & This study \\
\hline$r g f-3(P H-C N H)-s g f p$ & his- $3^{+}: \because P c c g-1-r g f-3(1077-1688)-s g f p$ & This study \\
\hline myc-rgf-3; $4 r g f-3$ & Pgpd-myc-rgf-3::nat ${ }^{R}$ rgf-3A::hph & This study \\
\hline$m y c-d b f-2^{T 67 I E}$ & Pgpd-myc-dbf-2 $2^{T 671 E}:: n a t^{R}$ & This study \\
\hline
\end{tabular}




\subsection{General molecular biological methods}

Standard methods were performed as described (Ausübel et al., 1997, Sambrook et al., 1989) or according to manufacturers instructions.

\subsubsection{Genomic DNA preparation and hybridisation}

$N$. crassa mycelia were harvested by filtration and ground in liquid nitrogen using mortar and pestle to obtain homogenized cell material. The powder was used to prepare genomic DNA with the Wizard ${ }^{\circledR}$ Genomic DNA Purification Kit (Promega GmbH, Madison, D) according to the Plant Tissue Protocol.

Southern blotting was performed as described before (Sambrook et al., 1989) using the Gene Images ${ }^{\mathrm{TM}}$ Random-Prime DNA labelling kit and the Gene Images ${ }^{\mathrm{TM}}$ CDP-Star ${ }^{\mathrm{TM}}$ Detection Kit (GE Healthcare Life Sciences, Munich, D) for non-radioactive labelling and detection.

\subsubsection{PCR}

Phusion ${ }^{\circledR}$ - (Finnzymes AG, Espoo, FIN) or Pfu-polymerase (Fermentas GmbH, St. Leon-Rot, D) were used for preparative PCR reactions according to manufacturers manuals. Alternatively control PCRs and colony PCRs were performed with Taq-polymerase (Fermentas GmbH, St. Leon-Rot, D). Custom oligonucleotides were ordered from Eurofins MWG Operon (Ebersberg, D) and Invitrogen GmbH (Karlsruhe, D). A standard PCR reaction consisted of an initial denaturation step over two minutes at $94^{\circ} \mathrm{C}, 30$ cycles with each cycle consisting of $30 \mathrm{~s}$ denaturation at $94^{\circ} \mathrm{C}, 30 \mathrm{~s}$ primer annealing at $50-60^{\circ} \mathrm{C}$ (depending on primer composition) and 1-7 min DNA elongation at $72^{\circ} \mathrm{C}$ (elongation time dependend on length of the to amplifying construct), and of a final elongation step at $72^{\circ} \mathrm{C}$ over $10 \mathrm{~min}$. Genomic DNA, cDNA or in case of colony PCRs E. coli transformants were used as template for the reactions. Site-directed mutagenesis PCRs or inverse PCRs were performed with the Phusion $^{\circledR}$ - (Finnzymes AG, Espoo, FIN) polymerase according to the QuickChange ${ }^{\mathrm{R}}$ SiteDirected Mutagenesis Kit protocol (Stratagen, La Jolla, USA). The obtained PCR products were digested with $D p n I$ to separate the new mutated non-methylated target plasmid from the non-mutated methylated input plasmid and subsequently transformed into E. coli. 


\subsubsection{DNA isolation}

DNA gel extraction was performed using the peqGOLD Gel Extraction Kit (PeqLab GmbH, Erlangen, D) or alternatively the QIAquick Gel Extraction Kit (Qiagen, Hilden, D) according to instruction manuals. Plasmid DNA from E. coli was prepared using peqGOLD Plasmid Miniprep Kit II (PeqLab GmbH, Erlangen, D).

\subsubsection{Ligation and restriction digests}

Ligation reactions were performed with T4 DNA ligase (Fermentas GmbH, St. Leon-Rot, D) in 2 fold concentrated quick ligation buffer (100 mM HEPES, pH 7.6, $20 \mathrm{mM} \mathrm{MgCl}$, $4 \mathrm{mM}$ DTT, $4 \mathrm{mM}$ ATP and 14\% (v/v) PEG4000) for 10 to $45 \mathrm{~min}$ at room temperature (RT). For subcloning PCR fragments were ligated into pJet1.2/blunt Cloning Vector of CloneJET ${ }^{\mathrm{TM}}$ PCR Cloning Kit (MBI Fermentas, St. Leon-Rot, D). Restriction enzymes were ordered from Fermentas (St. Leon-Rot, D) or New England Biolabs GmbH (Frankfurt, D) and digests were performed according to the instruction manuals.

\subsubsection{DNA transformation in $E$. coli}

Chemically competent $E$. coli cells were prepared and DNA transformation was performed as described (Inoue et al., 1990).

\subsubsection{Sequence analysis}

DNA was sequenced at the "Labor für Genomanalyse" (G2L, Göttingen, D). Sequences were analysed with 4Peaks (Copyright (C) 2001 - 2009: Mekentosj B.V. Gerberastraat 1171431 RA Aalsmeer, The Netherlands. KvK Amsterdam 34282675) and Lasergene (DNASTAR, Inc., Madison, USA) software. BLAST searches were performed at NCBI (http://www.ncbi.nlm.nih.gov/). For alignments MultAlin (Corpet, 1988; $\mathrm{http}$ ://bioinfo.genopoletoulouse.prd.fr/multalin/multalin.html) was used. Gene sequences of the investigated $N$. crassa genes were obtained by the $N$. crassa genome data base http://www.broadinstitute.org/annotation/genome/neurospora/MultiHome.html, for $A$. nidulans genes were available at http://www.broadinstitute.org/annotation/genome/aspergillus group/MultiHome.html. 


\subsection{Biochemical methods}

\subsubsection{Protein expression and purification in $E$. coli}

N. crassa Rho proteins RHO1 to RHO4, RAC and CDC42, the A. nidulans Rho GTPase AnRho4 (AN2687.3), the putative $N$. crassa Rho GEF domains of NCU06579 (amino acids 230-637) and NCU02131 (amino acids 811-1688) and the putative A. nidulans RhoGEF AnBud3 (AN0113.3) (amino acids 230-647) were expressed and purified as N-terminal MBP(maltose binding protein-) or GST- (glutathione S-transferase-) tagged fusion proteins from $E$. coli Rosetta2 (DE3) cells (Merck KGaA, Darmstadt, D). Freshly transformed cells were inoculated to an $\mathrm{OD}_{600}$ of 0.1 in $\mathrm{LB}^{+}$medium $(1 \% \mathrm{NaCl}, 0.8 \%$ yeast extract, $1.8 \%$ peptone, $0.4 \%$ glucose), grown at $20^{\circ} \mathrm{C}$ until an $\mathrm{OD}_{600}=0.4-0.5$ was reached and fusion protein expression was induced with $0.2 \mathrm{mM}$ IPTG for $2 \mathrm{~h}$. Subsequently cells were harvested by centrifugation and the pellet was resuspended in lysis buffer $(50 \mathrm{mM}$ Tris, $\mathrm{pH} 7.4,125 \mathrm{mM}$ $\mathrm{NaCl}, 5 \mathrm{mM} \mathrm{MgCl} 2,10 \%$ sucrose, 0.02\% NP-40, $2 \mathrm{mM}$ DTT, $1 \mathrm{mM} \mathrm{PMSF}, 0.35 \mathrm{mg} / \mathrm{ml}$ benzamidine) and stored at $-20^{\circ} \mathrm{C}$. Cell extracts were generated by ultrasonication and incubated either with preequilibrated Amylose Resin (New England Biolabs GmbH, Frankfurt, D) for MBP-tagged proteins or with preequilibrated GSH sepharose (Amersham Biosciences Europe GmbH, Freiburg, D) for GST-tagged proteins on a rotating wheel for one hour at $4^{\circ} \mathrm{C}$. Afterwards, the resin was washed twice with washing buffer $(50 \mathrm{mM}$ Tris, $\mathrm{pH}$ 7.4, $250 \mathrm{mM} \mathrm{NaCl}, 5 \mathrm{mM} \mathrm{MgCl} 2,10 \%$ glycerol, 0.02\% NP-40, $2 \mathrm{mM}$ DTT, $1 \mathrm{mM}$ PMSF, $0.35 \mathrm{mg} / \mathrm{ml}$ benzamidine) and fusion proteins were eluted in several steps with elution buffer (for MBP fusion proteins $=50 \mathrm{mM}$ Tris, $\mathrm{pH} 7.4,200 \mathrm{mM} \mathrm{NaCl}, 5 \mathrm{mM} \mathrm{MgCl} 2,10 \%$ glycerol, $0.02 \%$ NP-40, 2mM DTT, $20 \mathrm{mM}$ maltose and for GST fusion proteins $=50 \mathrm{mM}$ Tris, $\mathrm{pH} 7.4$, $200 \mathrm{mM} \mathrm{NaCl}, 5 \mathrm{mM} \mathrm{MgCl} 2,10 \%$ glycerol, $0.02 \%$ NP-40, $2 \mathrm{mM}$ DTT, $20 \mathrm{mM}$ Glutathione (red.)). Protein-rich fractions were pooled and protein concentrations were determined by Bradford analysis (Bradford, 1976). Protein expression was verified by Coomassie staining and/or Western blotting (see section 2.5.3).

\subsubsection{In vitro GEF assays}

Guanine nucleotide exchange assays were carried out by fluorometric determination of mant (2'(3')-O-methylanthraniloyl)-GDP incorporation as described (Abe et al., 2000) using a Tecan Infinite 200 spectrophotometer at $21^{\circ} \mathrm{C}$. Mant-GDP binding to purified Rho proteins leads to an increase in fluorescence intensity, since its fluorescence signal is no longer 
quenched in the aqueous solution. The reaction was started ( = point of time 0 ) by adding 0.1 $\mu \mathrm{M}$ mant-GDP and 0.8-1 $\mu \mathrm{M}$ MBP-GEF to 1-1.2 $\mu \mathrm{M}$ Rho GTPase in 30mM Tris, $\mathrm{pH} 7.5,5$ $\mathrm{mM} \mathrm{MgCl2,} 10 \mathrm{mM} \mathrm{NaH} 2 \mathrm{PO} 4 / \mathrm{K} 2 \mathrm{HPO} 4,3 \mathrm{mM}$ DTT, which was pre-equilibrated for $5 \mathrm{~min}$ at $21^{\circ} \mathrm{C}$. Fluorescence intensity $\left(\lambda_{\mathrm{exc}}=356 \mathrm{~nm}, \lambda_{\mathrm{em}}=448 \mathrm{~nm}\right)$ was monitored over $8 \mathrm{~min}$ and the resulting emission curves were further analyzed. To allow the comparison between independently prepared biological samples of GEF and GTPase, we used the linear range of the slope of each determined kinetic. The kinetics of at least two independent GEF and two independent GTPase preparations each performed in duplicate measurements were determined and the background fluorescence of mant-GDP without added proteins was subtracted. The mean value of the slopes calculated for the respective Rho protein in the absence of any GEF construct represents the intrinsic activity of the respective Rho protein and was set to $100 \%$. To determine the exchange activity of the Rho GTPase in the presence of the GEF relative to its intrinsic activity, the mean slope value calculated for emission curves of Rho in the presence of the GEF was divided by the mean slope value calculated for the intrinsic activity of Rho and the resulting value was multiplied by 100 to obtain the relative exchange activity of Rho in the presence of GEF. Relative exchange activity of the

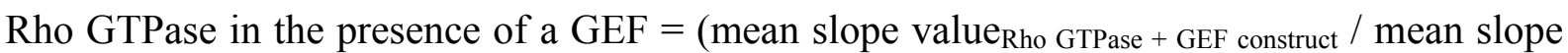
value Rho GTPase without GEF) $* 100$.

\subsubsection{Protein isolation and analysis}

To prepare crude cell extracts from $N$. crassa strains were grown in $50 \mathrm{ml}$ liquid minimal medium and mycelia were harvested by filtration. Subsequently cells were homogenized in protein extraction buffer $\left(100 \mathrm{mM} \mathrm{Na}_{2} \mathrm{HPO}_{4} \mathrm{pH} 7.6,50 \mathrm{mM} \mathrm{NaCl}, 1 \%\right.$ SDS $)$ by using the Precellys 24 (PeqLab GmbH, Erlangen, D; program: 3 x $15 \mathrm{sec}$ at $5500 \mathrm{rpm}$; 30sec pause). After centrifugation at $4^{\circ} \mathrm{C}$ for 10 min the supernatant was charged with $3 \times$ Laemmli buffer (10\% glycerol, $5 \% \beta$-mercaptoethanol, $15 \%$ SDS, $12,5 \%$ upper-buffer $(0,5 \mathrm{M}$ Tris- $\mathrm{HCl} \mathrm{pH}$ $6,8), 0,75 \%$ bromophenol blue, $3 \mathrm{M}$ urea) and incubated for $8 \mathrm{~min}$ at $98^{\circ} \mathrm{C}$. For Western hybridisation experiments proteins were separated by sodium dodecyl sulphate polyacrylamide gel electrophoresis (SDS-PAGE) and were transferred onto a nitrocellulose membrane by electroblotting (Ausübel et al., 1997). The PageRuler ${ }^{\mathrm{TM}}$ Prestained Protein Ladder (Fermentas GmbH, St. Leon-Rot, D) was used as marker. Mouse monoclonal Anti-cmyc antibody $9 E 10$ (Santa Cruz Biotechnology, Heidelberg, D), mouse monoclonal Anti-GFP (B-2): sc-9996 antibody (Santa Cruz Biotechnology, Heidelberg, D), monoclonal Anti-HA mouse clone HA-7 (Sigma-Aldrich, Taufkirchen, D) and Anti-MBP rabbit antiserum (New 
England Biolabs GmbH, Frankfurt, D) were used as primary antibodies followed by peroxidase-coupled goat anti-mouse or goat anti-rabbit $\mathrm{IgG}$ antibody (Invitrogen $\mathrm{GmbH}$, Karlsruhe, D) as secondary antibodies. Detection was performed using the ECL technology (GE Healthcare Life Sciences, München, D).

\subsubsection{Immunoprecipitation}

For immunoprecipitation the respective $N$. crassa strains were grown in $100 \mathrm{ml}$ liquid medium and mycelia were harvested by filtration and subsequently ground in liquid nitrogen. The powder was resuspended in lysis buffer $(20 \mathrm{mM}$ Tris $\mathrm{pH} 7.5,150 \mathrm{mM} \mathrm{NaCl}, 10 \%$ glycerol, $0.1 \%$ NP-40, $0.5 \mathrm{mM}$ EDTA, $1 \mathrm{mg} / \mathrm{ml}$ pepstatin A, $10 \mathrm{mg} / \mathrm{ml}$ aprotinin, $50 \mathrm{mg} / \mathrm{ml}$ benzamidine, $10 \mathrm{mg} / \mathrm{ml}$ leupeptin, $0.1 \mathrm{mM}$ PMSF, $1 \mathrm{M}$ DTT, $100 \mathrm{mM}$ sodium orthovanadate, $25 \mathrm{mM} \beta$-glycerophosphate, $100 \mathrm{mM} \mathrm{NaF}$ ) and the samples were centrifuged at $4000 \mathrm{~g}$ for 15 min at $4^{\circ} \mathrm{C}$. With the resulting supernatant a second centrifugation step at $22000 \mathrm{~g}$ for $30 \mathrm{~min}$ at $4^{\circ} \mathrm{C}$ was performed thereby obtaining a clear lysate. Afterwards, the cleared crude extract was incubated with either mouse monoclonal Anti-GFP (B-2): sc-9996 antibody (Santa Cruz Biotechnology, Heidelberg, D) for GFP-tagged proteins or with monoclonal Anti-HA mouse clone HA-7 (Sigma-Aldrich, Taufkirchen, D) for HA-tagged proteins on a rotation device for $2 \mathrm{~h}$ at $4^{\circ} \mathrm{C}$, and with Protein-A-Sepharose beads for additional $1 \mathrm{~h}$ at $4^{\circ} \mathrm{C}$. The beads were washed $3 \mathrm{x}$ with wash buffer (20 mM Tris $\mathrm{pH} 7.5,500 \mathrm{mM} \mathrm{NaCl}, 10 \%$ glycerol, $0.1 \% \mathrm{NP}-40$, $0.5 \mathrm{mM}$ EDTA, $1 \mathrm{mg} / \mathrm{ml}$ pepstatin A, $10 \mathrm{mg} / \mathrm{ml}$ aprotinin, $50 \mathrm{mg} / \mathrm{ml}$ benzamidine, $10 \mathrm{mg} / \mathrm{ml}$ leupeptin, $0.1 \mathrm{mM}$ PMSF, $1 \mathrm{M}$ DTT, $100 \mathrm{mM}$ sodium orthovanadate, $25 \mathrm{mM} \beta$ glycerophosphate, $100 \mathrm{mM} \mathrm{NaF}$ ). Immunoprecipitated proteins were recovered by boiling the beads for $10 \mathrm{~min}$ at $98^{\circ} \mathrm{C}$ in Laemmli buffer, separated by SDS-PAGE and stained with Coomassie (Merril, 1990). Alternatively, blotting and immunodetection were performed after gel electrophoresis.

\subsection{Microscopy}

Low magnification documentation of fungal hyphae or colonies was performed with a SZX16 stereomicroscope, equipped with a Colorview III camera and Cell ${ }^{\mathrm{D}}$ imaging software (Olympus, Tokyo, Japan). Images were further processed using Photoshop CS2 (Adobe, San Jose, USA). 


\subsubsection{Fluorescence microscopy}

For fluorescence microscopy of mature hyphae, strains were grown on 2-5 mm thick layers of Vogel's minimal medium (VMM) plates for 1-2 days, for microscopy of germinating conidia and young germlings, cultures were grown in liquid Vogel's minimal medium for 5-10 hours and for investigation of GFP expression in dormant conidia, conidia of the respective strains were dissolved in water. The "inverted agar block" method (Hickey et al., 2002) was used for live-cell imaging. For extended image acquisition, the excised agar blocks were not assembled on glass cover slips but in cell culture dishes with a glass bottom (Greiner Bio-One GmbH, Frickenhausen, D). Cell wall and plasma membrane were stained with Calcofluor White $(2 \mu \mathrm{g} / \mathrm{ml}$ dissolved in water; Sigma-Aldrich, Taufkirchen, D) and FM4-64 $(1 \mu \mathrm{g} / \mathrm{ml}$ dissolved in VMM); Molecular Probes, Eugene, USA), respectively. 2D microscopy was performed with an inverted Axiovert S100 microscope (Carl Zeiss AG, Oberkochen, Germany) equipped with an ORCA ER digital camera (Hamamatsu Photonics, Hamamatsu, Japan). Image acquisition was done using the Openlab 5.5 software (Improvision, Coventry, GB). For time-lapse imaging an inverted Axio Observer Z1 (Carl Zeiss AG, Oberkochen, Germany) microscope equipped with a QuantEM 512SC camera (Photometrics, Tucson, USA) was used. Time-lapse microscopy was performed at capture intervals of $20 \mathrm{~s}$ for periods up to $40 \mathrm{~min}$ using the oil immersion objective $100 \mathrm{x} / 1.3$. The slidebook 5.0 software (Intelligent Imaging Innovations, GmbH, Göttingen, Germany) was used for image acquisition, and image series were converted into movies ( ${ }^{*}$.mov) or into TIFF-series. 


\section{Results}

\subsection{Septation markers in Neurospora crassa}

To investigate which genes are involved in the septation process in Neurospora crassa, the available $N$. crassa knockout mutant library (Dunlap et al., 2007) was screened for deletion mutants that show defects in septum formation as indicated by cell lysis and lack of conidiation in developing colonies. In this screen we concentrated on genes that have homologues/orthologues in Saccharomyces cerevisiae or Schizosaccharomyces pombe known to be involved in cytokinesis/septation such as landmark proteins, regulators of the actin cytoskeleton, Rho proteins and their regulators or septins. Investigated candidate genes and their mutant phenotypes are summarized in Table 4. Three groups of mutants were classified according to their deletion phenotypes: (i) essential genes of which no viable ascospores or microconidia were obtained, (ii) aseptate mutants and (iii) mutants that were able to form septa and did not show obvious septation defect such as an altered septation pattern.

Table 4: Summary of $N$. crassa genes potentially involved in the septation process. For functionally characterized proteins/genes, the $N$. crassa names are used (lower-case letters), otherwise the names of $S$. cerevisiae homologues are given (capital letters). Genes were classified according to their putative functions and their respective deletion phenotype.

\begin{tabular}{|c|c|c|c|c|}
\hline $\begin{array}{c}\text { Functional } \\
\text { category }\end{array}$ & $\begin{array}{c}\text { no KO } \\
\text { available }\end{array}$ & $\begin{array}{c}\text { no viable ascospores } \\
\text { or microconidia } \\
\end{array}$ & aseptate & septa formed \\
\hline $\begin{array}{l}\text { Potential } \\
\text { landmark } \\
\text { proteins }\end{array}$ & & & $\begin{array}{l}\text { NCU06579 (BUD3) } \\
\text { NCU00152 (BUD4) }\end{array}$ & $\begin{array}{l}\text { NCU00481 }(A X L 1) \\
\text { NCU04601 }(A X L 2) \\
\text { NCU08343 }(R A X 1) \\
\text { NCU03045 }(R A X 2)\end{array}$ \\
\hline $\begin{array}{c}\text { Actin } \\
\text { organization }\end{array}$ & NCU04173 (act-1) & NCU01431 (bni-1) & $\begin{array}{l}\text { NCU03116 (IQG1) } \\
\text { NCU00551 (MYO2) } \\
\text { NCU06617 (MLC1) }\end{array}$ & \\
\hline $\begin{array}{c}\text { Rho } \\
\text { GTPases } \\
\text { and } \\
\text { RhoGEFs }\end{array}$ & & $\begin{array}{l}\text { NCU01484 (rho-1) } \\
\text { NCU00668 (ROM2) } \\
\text { NCU06067 }(c d c-24)\end{array}$ & $\begin{array}{l}\text { NCU03407 }(\text { rho-4) } \\
\text { NCU02131 }(R G F 3) \\
\text { NCU06579 (BUD3) }\end{array}$ & $\begin{array}{l}\text { NCU08683 }(\text { rho-2) } \\
\text { NCU00600 }(r h o-3) \\
\text { NCU02160 }(r a c-1) \\
\text { NCU06454 }(\text { cdc-42) } \\
\text { NCU02764 }(r s p-1) \\
\text { NCU09492 }(\text { DOCK) } \\
\text { NCU06561 }(r d i-1)\end{array}$ \\
\hline $\begin{array}{c}\text { Ras } \\
\text { GTPases } \\
\text { and } \\
\text { RasGEFs }\end{array}$ & & & & $\begin{array}{l}\text { NCU02167 (krev-1) } \\
\text { NCU03852 (BUD2) } \\
\text { NCU03616 (smco-7) } \\
\text { NCU06500 (CDC25) }\end{array}$ \\
\hline $\begin{array}{l}\text { Ste20-type } \\
\text { kinases }\end{array}$ & & & $\begin{array}{c}\text { NCU04096 } \\
(\text { SID1 in } S . \text { pombe })\end{array}$ & $\begin{array}{l}\text { NCU00406 (CLA4) } \\
\text { NCU03894 (STE20) } \\
\text { NCU11235 (pod-6) } \\
\text { NCU00772 (gck-1) }\end{array}$ \\
\hline
\end{tabular}


Table 4 (continued): Summary of $N$. crassa genes potentially involved in the septation process. For functionally characterized proteins/genes, the $N$. crassa names are used (lower-case letters), otherwise the names of $S$. cerevisiae homologues are given (capital letters). Genes were classified according to their putative functions and their respective deletion phenotype.

\begin{tabular}{|c|c|c|c|c|}
\hline $\begin{array}{l}\text { Functional } \\
\text { category }\end{array}$ & no KO available & $\begin{array}{c}\text { no viable } \\
\text { ascospores or } \\
\text { microconidia }\end{array}$ & aseptate & septa formed \\
\hline $\begin{array}{l}\text { Septation } \\
\text { initiation } \\
\text { network }\end{array}$ & NCU08878 (TEM1) & & $\begin{array}{l}\text { NCU01605 (mob-1) } \\
\text { NCU09071 (dbf-2) } \\
\text { NCU01335 (CDC15) }\end{array}$ & NCU06636 (CDC14) \\
\hline Septins & & NCU03795 (CDC12) & & $\begin{array}{l}\text { NCU08297 (CDC3) } \\
\text { NCU03515 (CDC10) } \\
\text { NCU02464 (CDC11) }\end{array}$ \\
\hline Polarisome & & NCU01431 (bni-l) & & $\begin{array}{l}\text { NCU08468 (BUD6) } \\
\text { NCU03115 (SPA2) }\end{array}$ \\
\hline $\begin{array}{l}\text { Other } \\
\text { candidates }\end{array}$ & NCU10905 (HOF1) & & NCU04095 (CYK3) & NCU06648 (BOI1) \\
\hline Cell wall & & & & $\begin{array}{l}\text { NCU03611 (CHS1) } \\
\text { NCU05239 (CHS2) } \\
\text { NCU04251 (CHS3) } \\
\text { NCU09324 (CHS4) } \\
\text { NCU04352 (CHS5) } \\
\text { NCU05268 (CHS6) } \\
\text { NCU05350 (CHS7) } \\
\text { NCU07435 (CHS5) } \\
\text { NCU05411 (BUD7) } \\
\text { NCU05720 (CHS7) }\end{array}$ \\
\hline
\end{tabular}

The following genes were found to be essential in $N$. crassa so that a function in septation cannot be excluded: the sole formin-coding gene present in $N$. crassa bni-1, the Rho GTPase rho-1, the Rho GEFs NCU00668 and $c d c-24$ and the septin NCU03795. Mutants of the putative GEF and landmark protein NCU06579, of the homologue of bud-4 NCU00152, of the actin cytoskeleton regulators igg-1, myo-2 and mlc-1, of the Rho GTPase rho-4, of the putative Rho GEF NCU02131, of the Ste20-like kinase NCU04096, of the homologues of septation initiation network genes $d b f-2$, mob-1 and $c d c-7$ (NCU09071, NCU01605 and NCU01395), and of the homologue of cyk-3 NCU04095 displayed an aseptate phenotype. The inability of these mutants to form septa was further confirmed by fluorescence microscopy using Calcofluor White staining. In the following, the functions of NCU02131, NCU06579 and NCU00152 in the septation process were further characterized. 


\subsection{The two distinct exchange factors BUD3 and RGF3 control RHO4- dependent septum formation}

C. Rasmussen showed in her work that the Rho GTPase RHO4 is required for septation in N. crassa. In the mutant screen for further proteins involved in this process the two putative GEFs NCU06579 and NCU02131 were identified as essential for septum formation. Furthermore, both mutants displayed the same defects as described for rho-4, such as complete lack of septa, slow growth (ca. 15\% of wild type), abnormal branching, thin hyphae, cytoplasmic leakage and abolished conidiation (Fig. 4), suggesting the two GEFs may act as RHO4-specific GEFs.

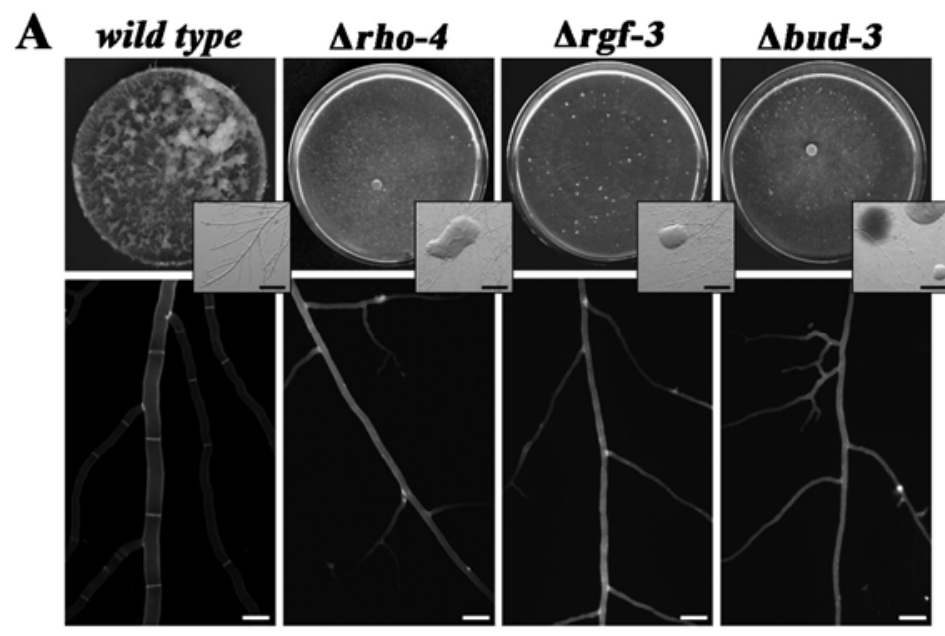

B

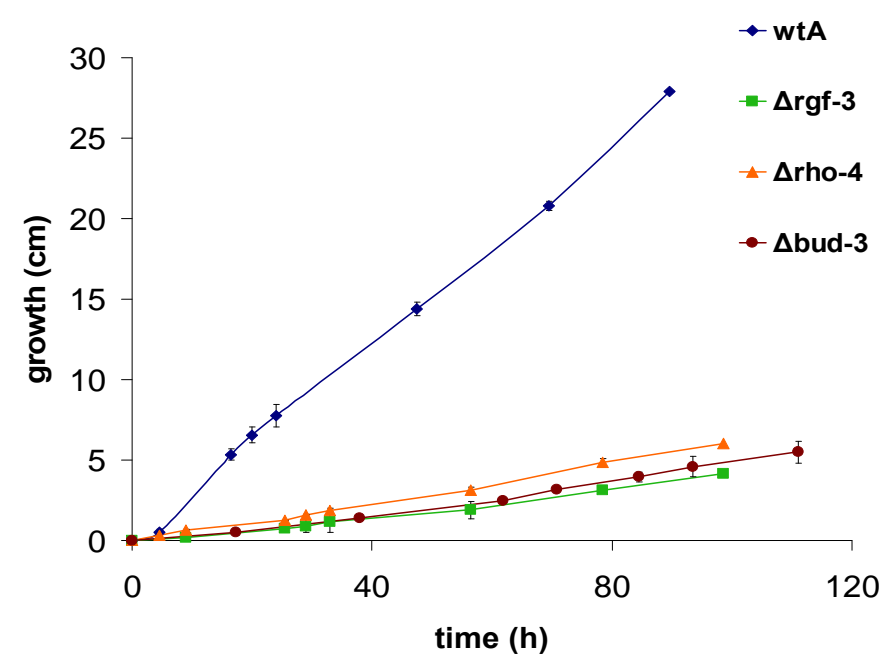

Fig. 4: Septum formation is controlled by RHO4, BUD3 and RGF3. (A) Phenotypic characterization of the indicated $N$. crassa strains. Note the inability to conidiate, the leakage of cytoplasm (magnified inserts; bars = $500 \mu \mathrm{m})$ and the lack of septa in the $\Delta r h o-4, \Delta r g f-3$ and $\Delta b u d-3$ deletion strains. Furthermore, these mutants form thin hyphae and show an abnormal branching pattern. The cell wall and septa are labelled with Calcofluor White. Bars $=10 \mu \mathrm{m}$. (B) $\Delta r h o-4, \Delta r g f-3$ and $\Delta b u d-3$ display a slow-growth phenotype. While the wild type grows at a rate of $0,3 \mathrm{~cm} / \mathrm{h}$, the growth rates of the mutants are between 0,03 and $0,06 \mathrm{~cm} / \mathrm{h}$. 
Complementation of the mutant defects either by introducing the full-length gef-gfp fusion constructs pMF272_rgf-3 and pMF272_bud-3_nat (also see next section) or the full-length over expression construct pEHN1_nat_myc_rgf-3 into the respective deletion strain validated the specificity of the defects for the two deletions. NCU06579 and NCU02131 encode for proteins with a predicted size of $167 \mathrm{kDa}$ and $186 \mathrm{kDa}$, respectively, and are characterized by a domain structure also found in other GEFs (Fig. 5). Both proteins have a centrally located Rho GEF domain also called $\underline{\mathrm{d}} b 1$ homology (DH) domain (amino acids 254-461 and 8671054, respectively). In addition, in NCU02131 a pleckstrin homology (PH) domain (amino acids 1091-1258) and a citron homology (CNH) domain (amino acids 1329-1634) are present. $\mathrm{PH}$ domains mediate protein and/or lipid interactions, while $\mathrm{CNH}$ domains are thought to have regulatory functions and may be involved in macromolecular interactions. Sequence analysis revealed that among functionally characterized proteins, AgBud3p and SpRgf3 from Ashbya gossypii and Schizosaccharomyces pombe, respectively, are the closest relatives of NCU06579 and NCU02131 (expect values of $7 \mathrm{e}^{-06}$ and $2 \mathrm{e}^{-103}$, respectively), and thus the two N. crassa proteins were named accordingly.

A

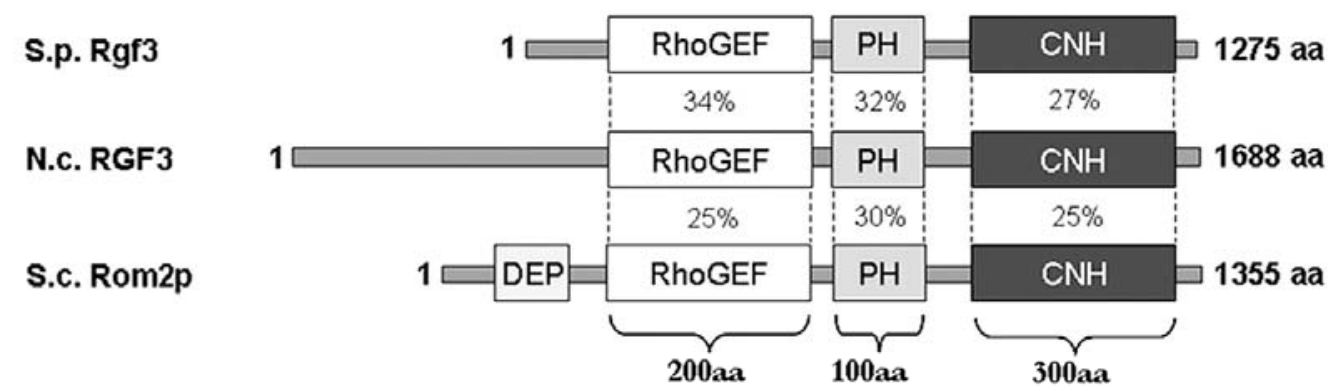

B

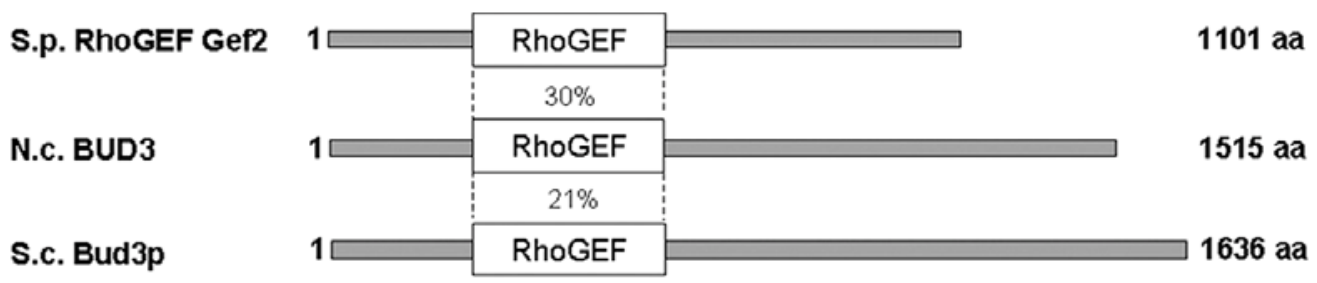

Fig. 5 Domain structure and sequence comparison of the $N$. crassa GEFs RGF3 (A) and BUD3 (B) with homologous proteins from budding and fission yeast. Sequence similarities between the domains in the three proteins are given in per cent; RhoGEF: catalytic domain, also called Dbl-homologous (DH) domain; PH: pleckstrin homology domain; DEP: domain found in Dishevelled, Egl-10, and Pleckstrin; CNH: citron homology domain.

No enzymatic function for $A$. gossypii and $S$. cerevisiae Bud3p or to Gef2, the closest, but uncharacterized $S$. pombe homologue of NCU06579/BUD3 (expect value of $3 \mathrm{e}^{-14}$ ), was 
described so far. Only a weak similarity to Rho-GEFs was noted for A. gossypii Bud3p (Wendland, 2003). In contrast, a clear role during septum formation has been assigned for SpRgf3, the closest S. pombe homologue of NCU02131/RGF3 (Morrell-Falvey et al., 2005, Mutoh et al., 2005). It was described as a Rhol interacting protein in fission yeast (Tajadura et al., 2004). Similarly, Rom2p and Rom1p, the closest NCU02131/RGF3 homologues in $S$. cerevisiae (expect values of $5 \mathrm{e}^{-25}$ and $4 \mathrm{e}^{-22}$, respectively), are GEFs that are specific for Rho1p (Ozaki et al., 1996, Park \& Bi, 2007).

In order to investigate, if RGF3 and BUD3 function as GEFs in N. crassa, and if so, which of the six Rho GTPases present in $N$. crassa are regulated by them, in vitro GEF assays were performed. For these assays full-length-cDNAs of the six Rho proteins RHO1, RHO2, RHO3, RHO4, CDC42 and RAC and cDNA fragments of BUD3 and RGF3 containing the catalytic GEF domain and the GEF, PH and CNH domains respectively, were generated and expressed as MBP or GST fusion proteins in E. coli. The six Rho proteins could be purified in sufficient amounts and all showed an intrinsic GDP-GTP exchange activity in the assays, indicating that the purified proteins were functional. During the cloning of the $r g f-3 \mathrm{cDNA}$ the existence of two splice variants of RGF3 was noted that differed in the presence of an additional intron within the PH domain. These two splice variants RGF3_short (amino acids 811-1672) and RGF3_long (amino acids 811-1688) were bacterially expressed and purified. The yield of purified MBP-tagged RFG3_short and RGF3_long with a size of 135-145kDa was low and the proteins seemed to be degraded into smaller products of $100-120 \mathrm{kDa}$ (Fig. 6). However, the quantity was sufficient to perform in vitro GEF assays, and both purified variants displayed GEF activity on RHO4 in the assays.

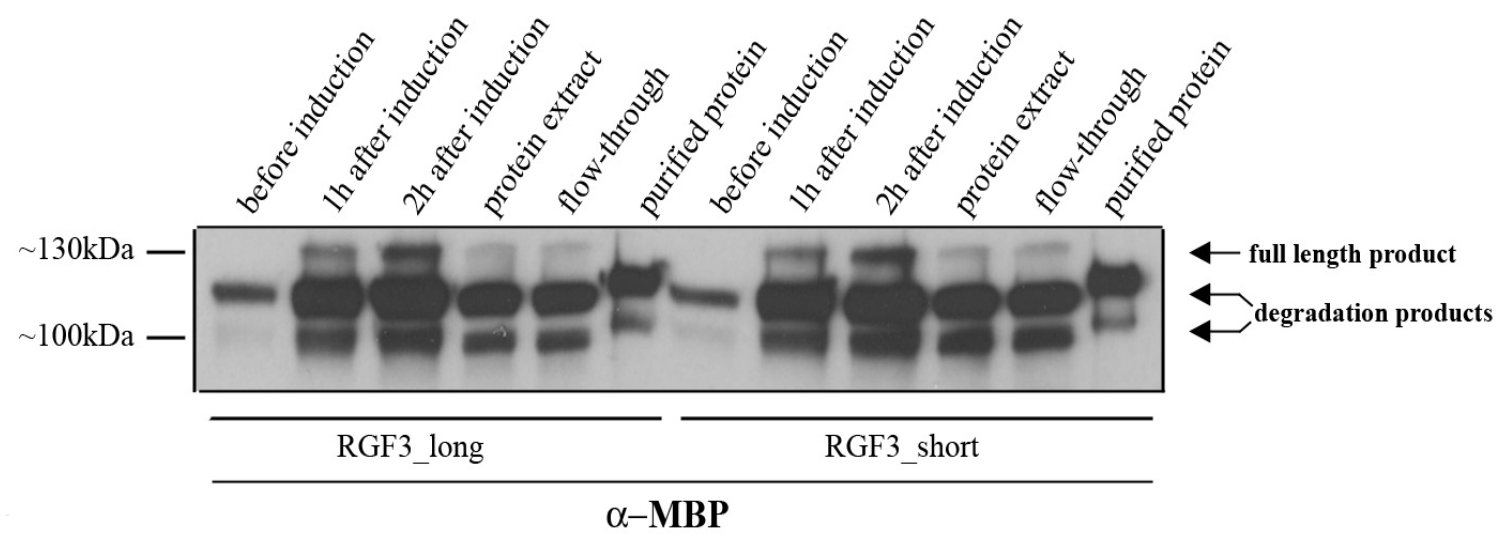

Fig. 6: Expression and purification of the two MBP-tagged RGF3 splice variants RGF3 long and RGF3_short are verified by Western blot analysis using MBP antibodies. Expression of both MBP-RGF3 fusion constructs was induced in $E$. coli for two hours (indicated by the appearance of a band at the predicted size of $130 \mathrm{kDa}$ and two lower bands representing degradation products). Subsequently, the fusion proteins were successfully affinity-purified using amylose resin. 
Interestingly, the two RGF3 versions showed no differences in their substrate specificity in vitro. RGF3_short as well as RGF3_long specifically stimulated the GDP-GTP exchange activity of purified RHO4 (Fig. 7B, C). The exchange activity of the other five GTPases however, was not stimulated by addition of RGF3_short or RGF3_long (Fig. 7B, C). Although both RGF3 variants showed the same substrate specificities in vitro, they may still add to the complexity of RHO4 signalling in vivo. Corresponding GEF assays of E. coliexpressed BUD3-MBP were performed by C. Richthammer (Institute of Microbiology and Genetics, University of Göttingen, D). BUD3 also stimulated the GDP-GTP exchange activity of RHO4, but of no other Rho GTPase (Fig. 8).

Thus, the mutant characteristics and the in vitro experiments identified BUD3 and RGF3 as RHO4-specific GEFs in N. crassa. In addition, N. crassa mutants in the Rho GTPases rho-2, rho-3, rac and $c d c-42$ displayed distinct defects and were still able to generate septa (data not shown), while rho- 1 is an essential gene (Vogt, 2008), further supporting the specificity of RGF3 and BUD3 for RHO4. 

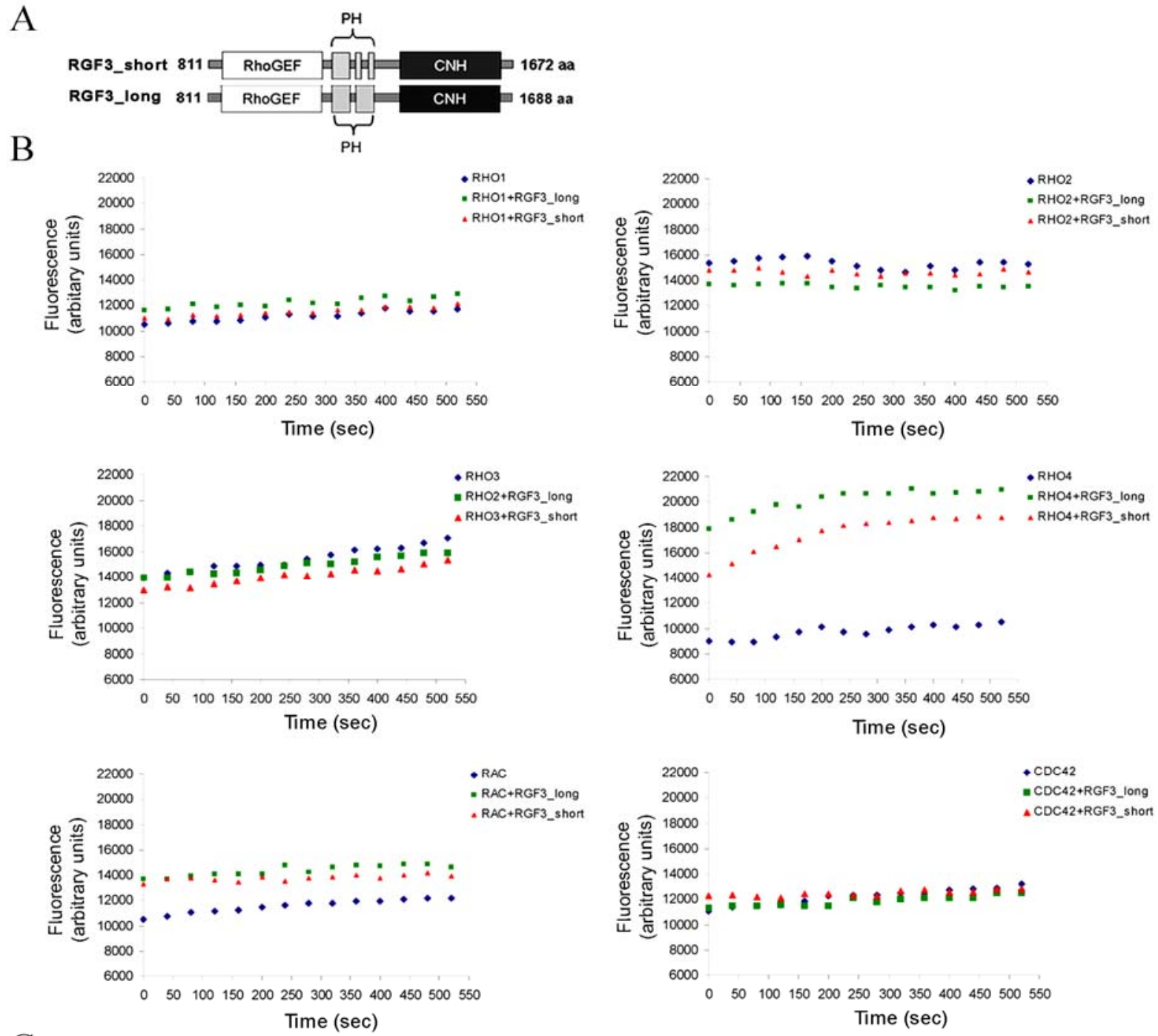

C

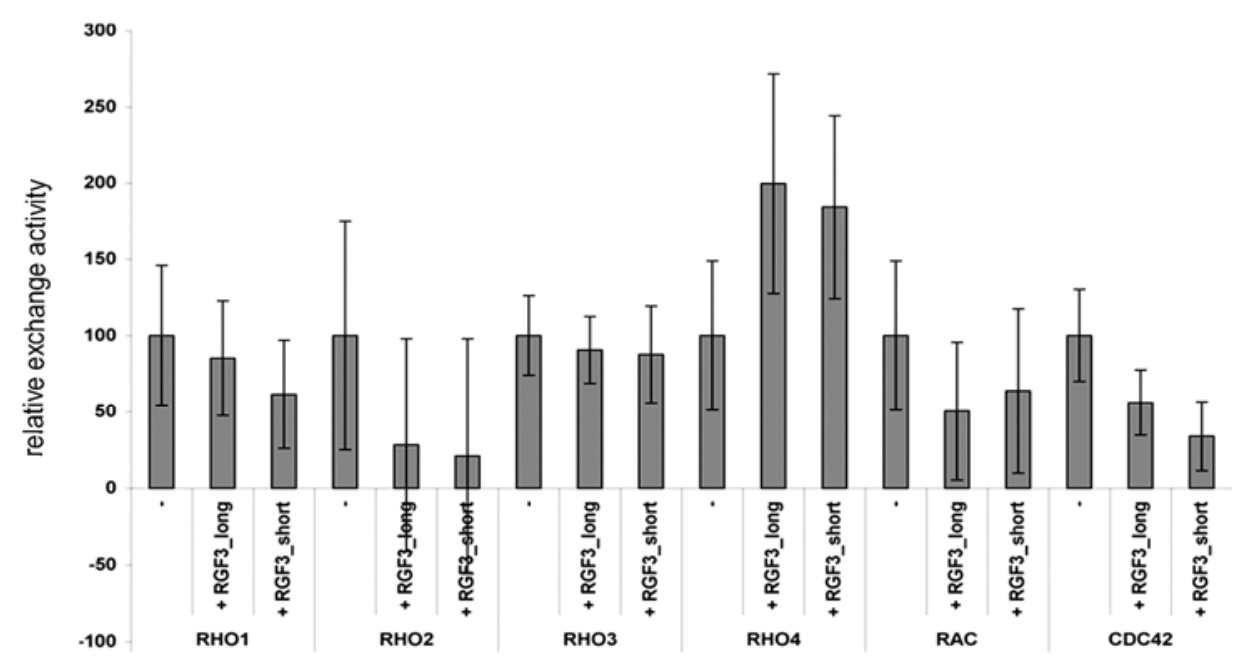

Fig. 7: RGF3 is a RHO4-specific GEF. (A) Schematic drawing of the RGF3 domains used for the in vitro GEF assays. (B) Exemplary in vitro kinetics of fluorescent mant-GDP binding to the six Rho GTPases present in $N$. crassa in the absence/presence of RGF3 short and RGF3 long are shown. Both RGF3 variants specifically stimulated the in vitro exchange activity of RHO4, but not of any of the other five Rho GTPases. Activity is given in arbitrary fluorescence units. (C) Summary of the relative exchange activity of the Rho proteins in the presence of RGF3_long and RGF3_short. The mean values \pm SD of at least two independent Rho and two GEF purifications with each experiment performed in duplicates were calculated and the intrinsic Rho activity was set to $100 \%$. 
A

BUD3 $230 \rightleftharpoons 637$ aa

$\mathrm{B}$
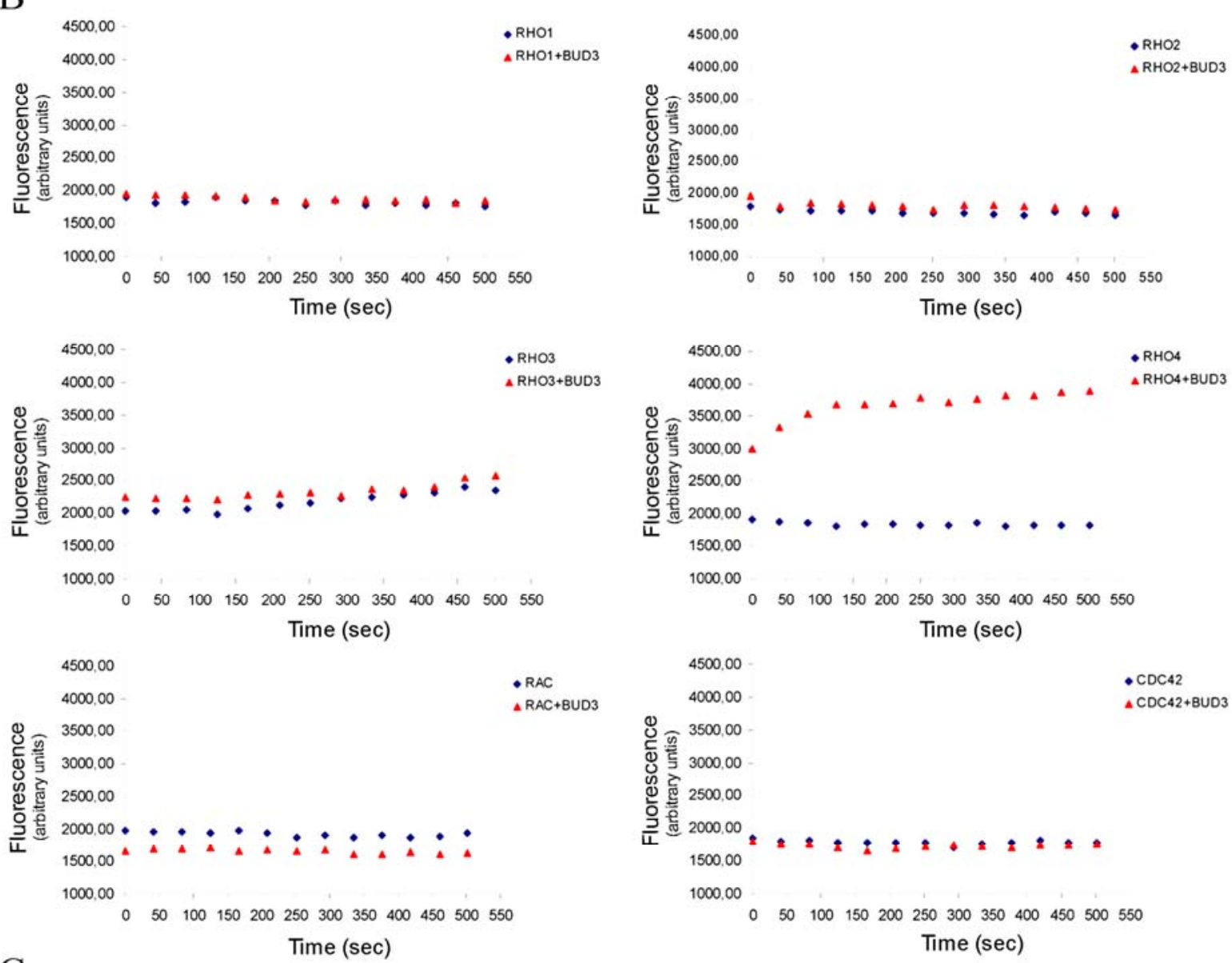

$\mathrm{C}$

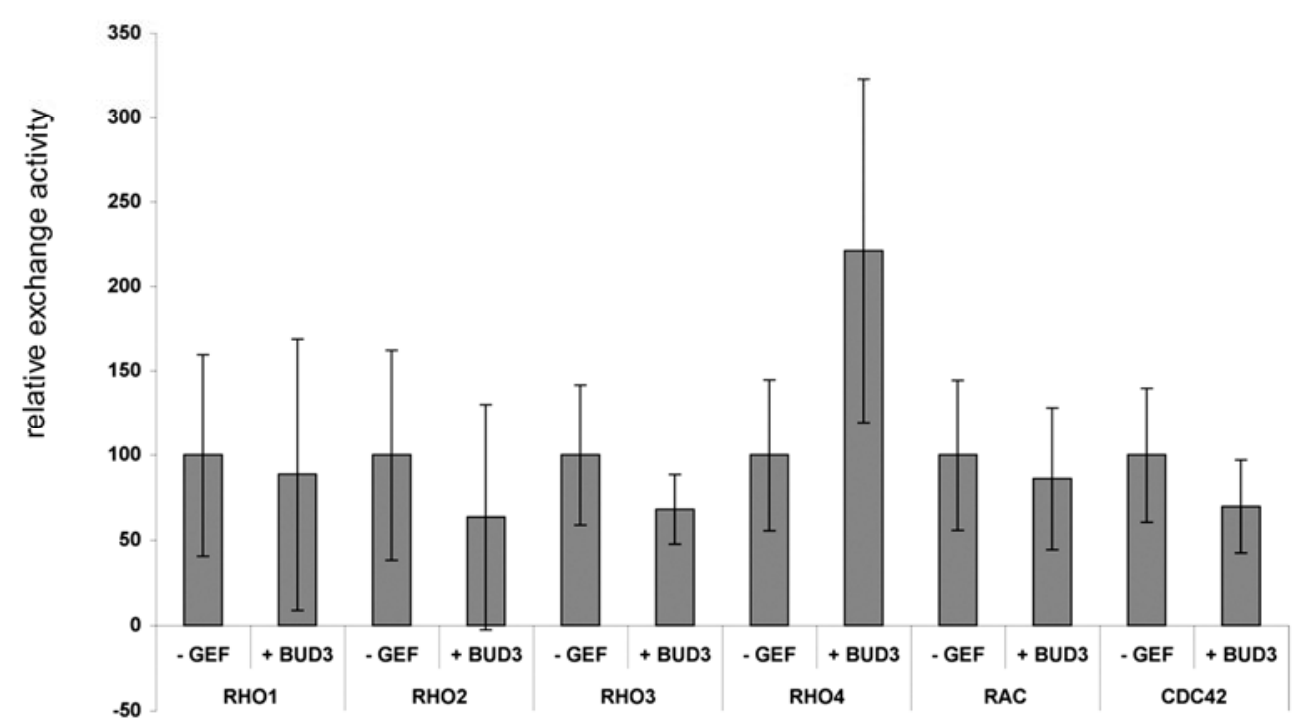

Fig. 8: BUD3 is a RHO4-specific GEF. (A) Schematic drawing of the BUD3 construct used for the in vitro GEF assays. (B) Exemplary in vitro kinetics of fluorescent mant-GDP binding to the six Rho GTPases present in $N$. crassa in the absence/presence of BUD3 are shown. BUD3 specifically stimulated the in vitro exchange activity of RHO4, but not of any of the other five Rho GTPases. Activity is given in arbitrary fluorescence units. (C) Summary of the relative exchange activity of the Rho proteins in the presence/absence of BUD3. The mean values $\pm \mathrm{SD}$ of at least two independent Rho and two GEF purifications with each experiment performed in duplicates were calculated and the intrinsic Rho activity was set to $100 \%$. 


\subsection{Aspergillus nidulans AnBud3 acts as an AnRho4 GEF and can also stimulate $N$. crassa RHO4 activity}

After BUD3 was shown to act as a RHO4-specific GEF in N. crassa, I wanted to investigate if BUD3 and its function as a RHO4 GEF is conserved within filamentous fungi. For this purpose I screened the Aspergillus nidulans genome database for homologues of BUD3 and RHO4 on the basis of sequence similarities. In this screen A. nidulans homologues of $N$. crassa BUD3 (encoded by the gene AN0113; expect value of 0.0 between $N$. crassa BUD3 and AN0113), and of N. crassa RHO4 (encoded by the gene AN2687; expect value of $8 \mathrm{e}^{-90}$ between $N$. crassa RHO4 and AN2687), were identified and named accordingly. To determine if AnBud3 also acts as a GEF and stimulates the GDP-GTP exchange activity of AnRho4, in vitro GEF assays were performed. Therefore, full-length cDNA of An-rho-4 (amino acids 1-280) and a cDNA fragment of An-bud-3 containing the catalytic RhoGEF domain (amino acids 230-647) were cloned and sequenced. The cDNA fragment of An-bud-3 contained no introns. Sequencing of the An-rho-4 cDNA revealed the existence of three exons disrupted by two introns with a length of $45 \mathrm{bp}$ and $41 \mathrm{bp}$ at positions +2998 and +3248 relative to the start codon, respectively. These data confirmed the in silico predictions, except for the second intron in An-rho-4, which was shortened by one codon in vivo. An-bud-3 and An-rho-4 cDNAs were used to express MBP-fusion proteins in E. coli, which were affinitypurified using amylose resin. High yields of both proteins with predicted sizes of $86 \mathrm{kDa}$ for AnBud3 and $71 \mathrm{kDa}$ for AnRho4 were obtained (Fig. 9) and in vitro GEF assays were performed (Fig. 10).

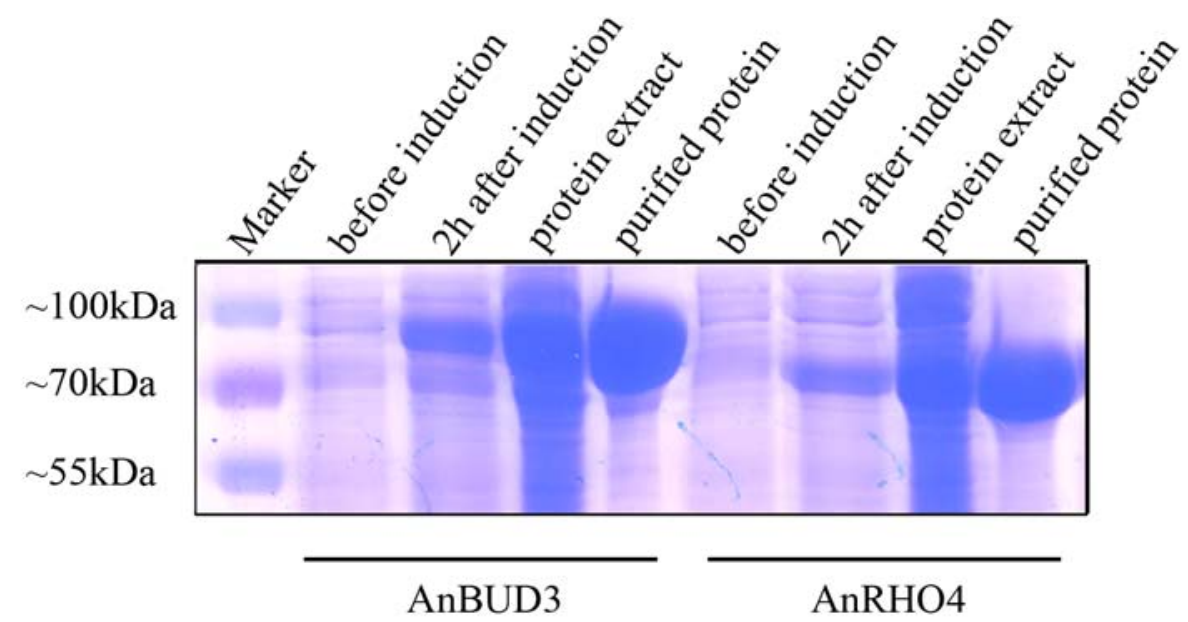

Fig. 9: Coomassie staining of bacterially expressed and affinity-purified full-length MBP tagged AnRho4 (71 kDa) and MBP tagged AnBud3 (86kDa) construct. Both constructs were expressed in E. coli for two hours as indicated by the appearance of a band at the predicted size. Subsequently both proteins were affinity-purified using amylose resin. 
AnBud3 stimulated the GDP-GTP exchange activity of AnRho4 in the in vitro assays (Fig. 10). Interestingly, AnBud3 was also able to stimulate the exchange activity of purified Neurospora crassa RHO4 in vitro (Fig. 10A,B), suggesting a highly conserved nature for the activation of Rho GTPases through their respective GEFs across these two fungal species.

A
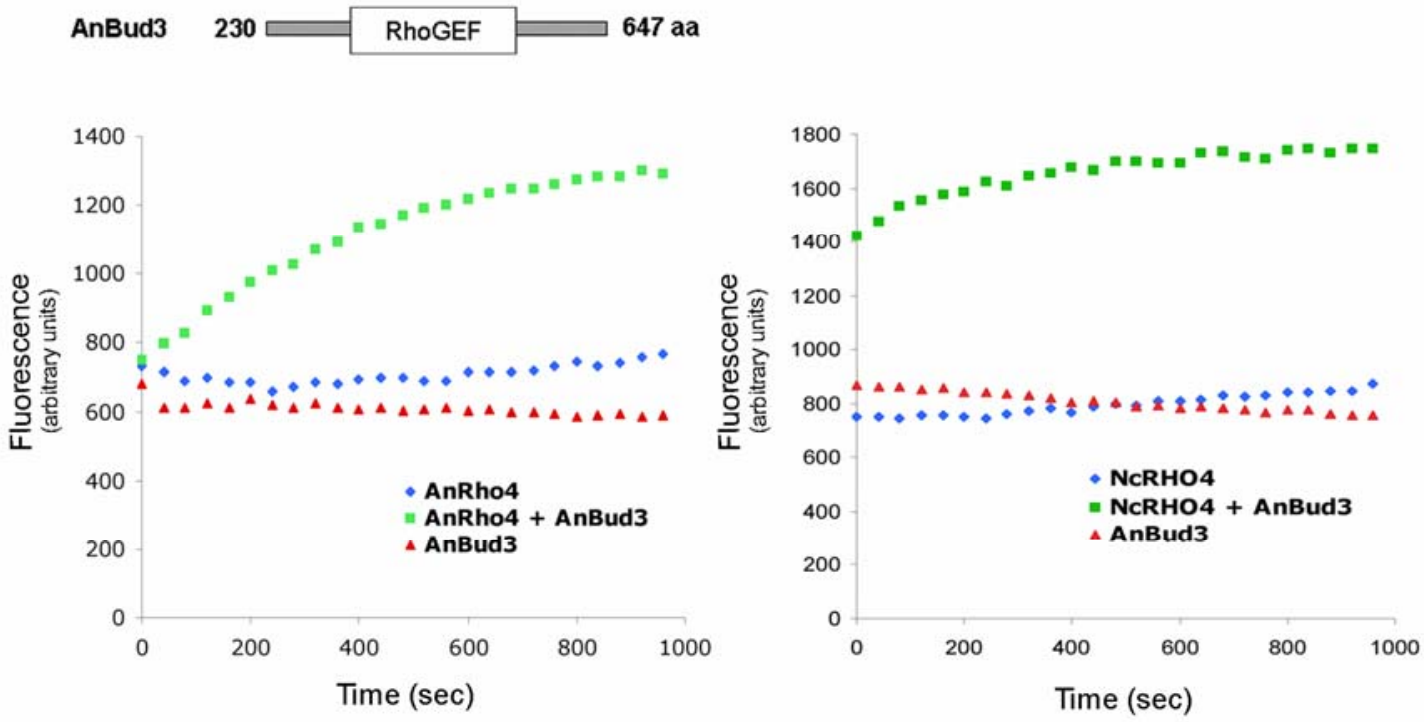

B

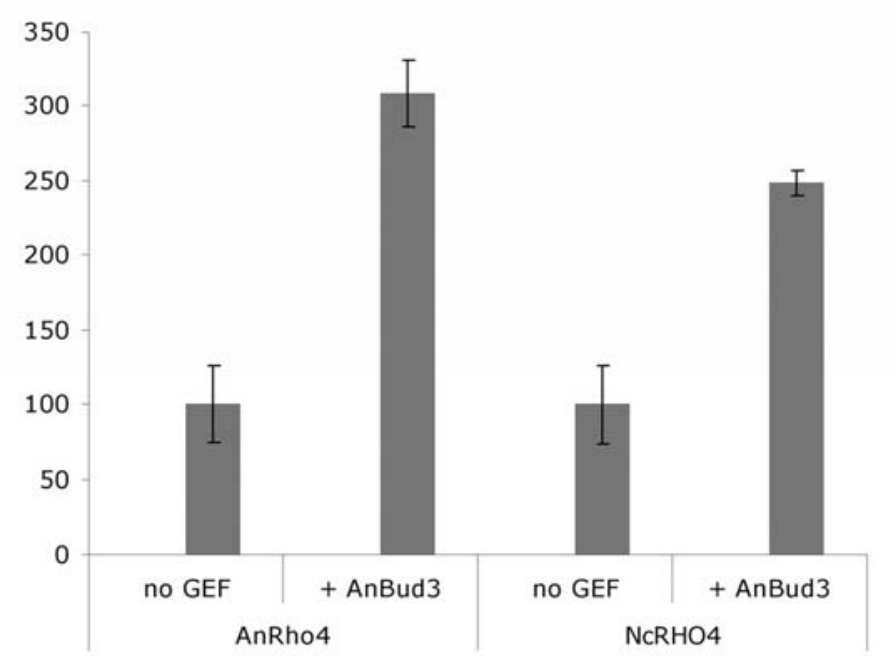

Fig. 10: AnBud3 is a RHO4 GEF. (A) Schematic drawing of the AnBud3 fusion protein used for the in vitro GEF assays and exemplary kinetics of AnBud3 induced mant-GDP binding to AnRho4 and NcRHO4, respectively. The in vitro guanine nucleotide exchange activity was determined by measuring binding of fluorescent mant-GDP to purified AnRho4 or NcRHO4 in the presence or absence of the AnBud3 construct containing the GEF domain. Activity is given in arbitrary fluorescence units. (B) Summary of the relative exchange activity of AnRho4 and NcRHO4 in the presence/absence of AnBud3. AnBud3 stimulated the exchange activity of AnRho4 as well as of NcRHO4. The diagram indicates the mean values $\pm \mathrm{SD}$ of at least two independent Rho protein and two GEF purifications with each experiment performed in duplicates. The intrinsic Rho activity is set to $100 \%$. 


\subsection{BUD3 and RGF3 define two independent functions of RHO4 during septation}

RGF3 and BUD3 functioned as RHO4-specific GEFs in N. crassa in vitro. Next I determined the localization of BUD3 and RGF3 in the fungus to investigate if both proteins show localization patterns similar to that described for RHO4 (Rasmussen \& Glass, 2007). Therefore functional GEF-GFP fusion proteins integrated at the his-3 locus under the control of the $c c g-1$ promoter in the respective deletion strain were generated. The $c c g-1$ promoter is repressed by glucose and during vegetative growth (Freitag et al., 2004) and is frequently used for the expression of the fusion proteins at low levels in N. crassa (e.g. it was utilized for RHO4 expression in the localization studies of Rasmussen \& Glass, 2007 and for expression of signalling molecules such as SOFT and MAK2 (Fleissner et al., 2009, Honda \& Selker, 2009)). Although the same expression system was used for both fusion constructs, protein levels of BUD3-GFP and RGF3-GFP were distinct (Fig. 11). While I was able to detect RGF3-GFP in cell extracts, BUD3-GFP was only detectable after immunoprecipitation.

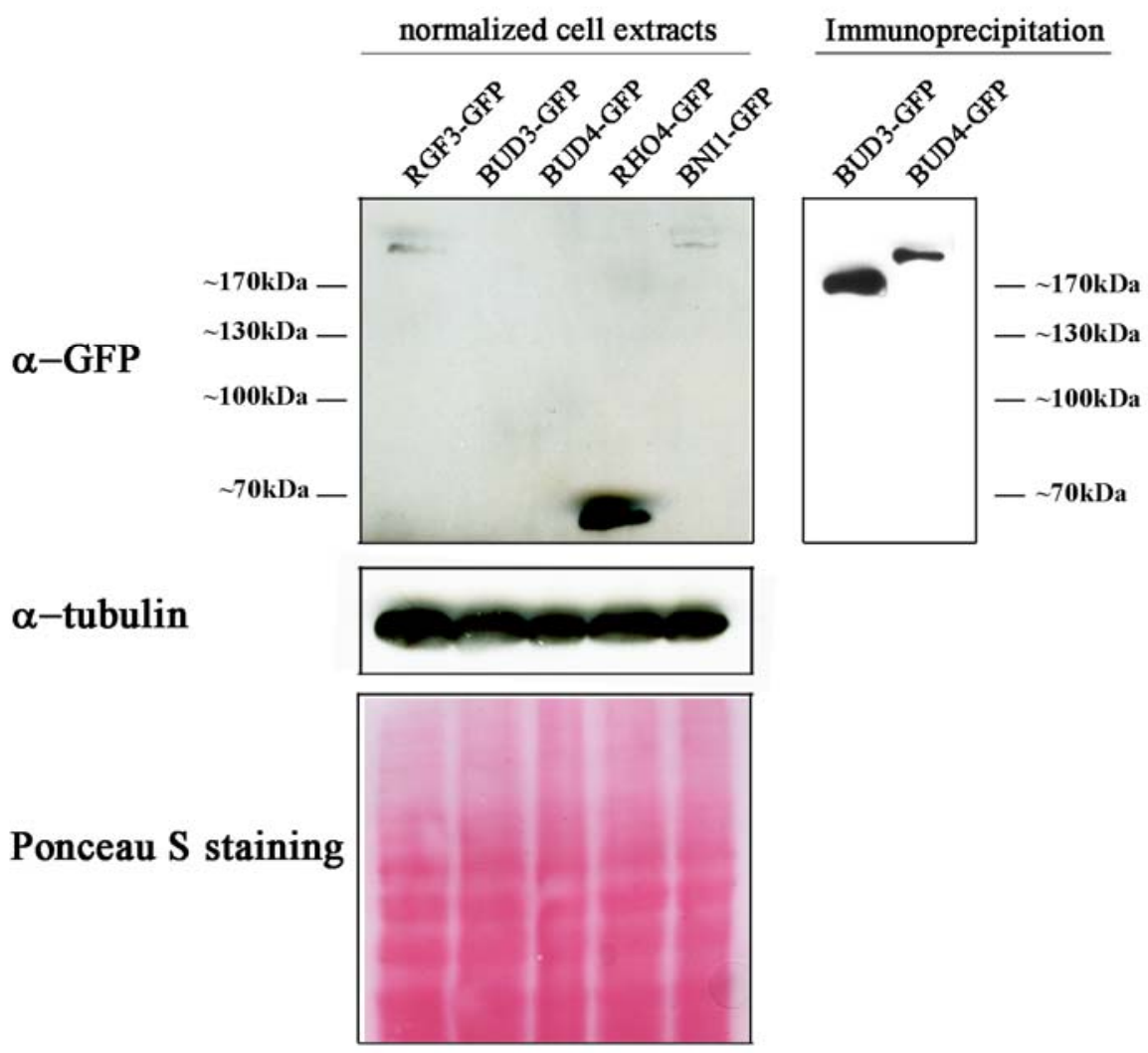

Fig. 11: Protein levels of RGF3-GFP, BUD3-GFP, BUD4-GFP, RHO4-GFP and BNI1-GFP are distinct although the same expression system is used. GFP fusion proteins of RGF3 (214kDa), RHO4 (65kDa) and BNI1 $(230 \mathrm{kDa})$ are detectable in cell extracts by Western blot analysis using anti-GFP antibodies, whereas BUD3-GFP and BUD4-GFP are only detected after immunoprecipitation with anti-GFP antibodies. Normalization of cell extracts is confirmed by Ponceau S staining and Western blot against tubulin. 
Both GEF fusion proteins complemented the respective mutants and localized as cortical ring prior to or concomitant with septation initiation, which was monitored by Calcofluor White or FM4-64 staining of the cell wall or plasma membrane, respectively. Furthermore, they localized as one constricting ring to the developing septum (Fig. 12A, B; Fig. 19A, B) as described for RHO4 (Rasmussen \& Glass, 2007; Fig. 19D). Interestingly, BUD3, but not RGF3, persisted around the mature septal pore after completion of septation in a manner similar to that observed for RHO4 (Rasmussen \& Glass, 2007; Fig. 19D). Exemplary TIFF series of time course experiments with RGF3-GFP, BUD3-GFP and RHO4-GFP are shown in Fig. 19A, B and D at the end of section 3.6.

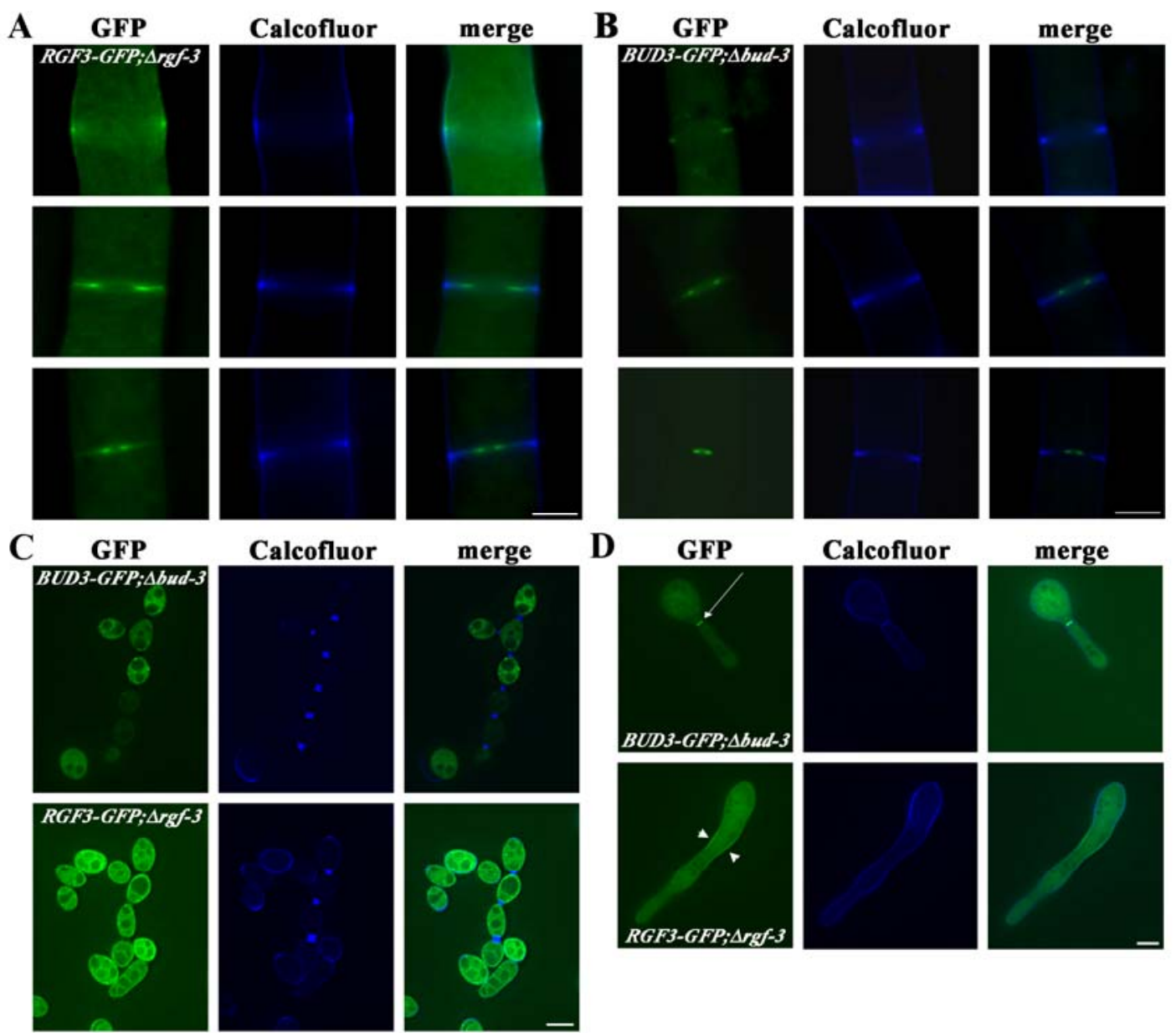

Fig. 12: BUD3 and RGF3 localize to the forming septum, but not to the hyphal tip. (A) RGF3-GFP and (B) BUD3-GFP accumulate at incipient septation sites and form constricting rings in vegetative hyphae. TIFF series of RGF3-GFP and BUD3-GFP localization during septum formation are shown in Fig. 19A and B, respectively. Note that BUD3-GFP, but not RGF3-GFP remains at the septal pore of mature septa. (C) RGF3-GFP and BUD3-GFP are not distributed in a polarized manner in dormant conidiospores. (D) Both GEF-GFP fusion proteins show no apical localization in young germlings. The arrow indicates a septum in the germling that is labelled by BUD3-GFP. Note that RGF3-GFP fluorescence is also observed in the cytoplasm and along the plasma membrane (arrow heads). Cell wall and septa are stained with Calcofluor White. Bars $=5 \mu \mathrm{m}$.

To investigate if RGF3 and BUD3 are involved in polarity establishment or maintenance, the localization pattern of both GFP fusion proteins in dormant and germinating conidiospores 
was analyzed (Fig. 12C, D). RGF3 localized diffusely throughout the cytoplasm and along the plasma membrane in dormant conidia, a localization pattern that was also described for RHO4 (Rasmussen \& Glass, 2007). In contrast, BUD3 localized in a patchy and vesicular manner through the cytoplasm and did not accumulate along the plasma membrane. This difference was also observed during germination. While BUD3 was only detectable at forming septa, RGF3 in addition also localized to the plasma membrane. Both proteins did not accumulate at the point of future germ tube emergence, at the tip of young germ tubes or mature hyphal tips, indicating that they have no prominent role in polarity establishment and extended polar tip growth, a notion that is consistent with the observed mutant characteristics.

To investigate if the localization of both GEFs is dependent on RHO4, the distribution pattern of BUD3-GFP and RGF3-GFP in a rho-4(RIP) background lacking functional RHO4 (Rasmussen \& Glass, 2005), where no septa are formed, was analyzed.
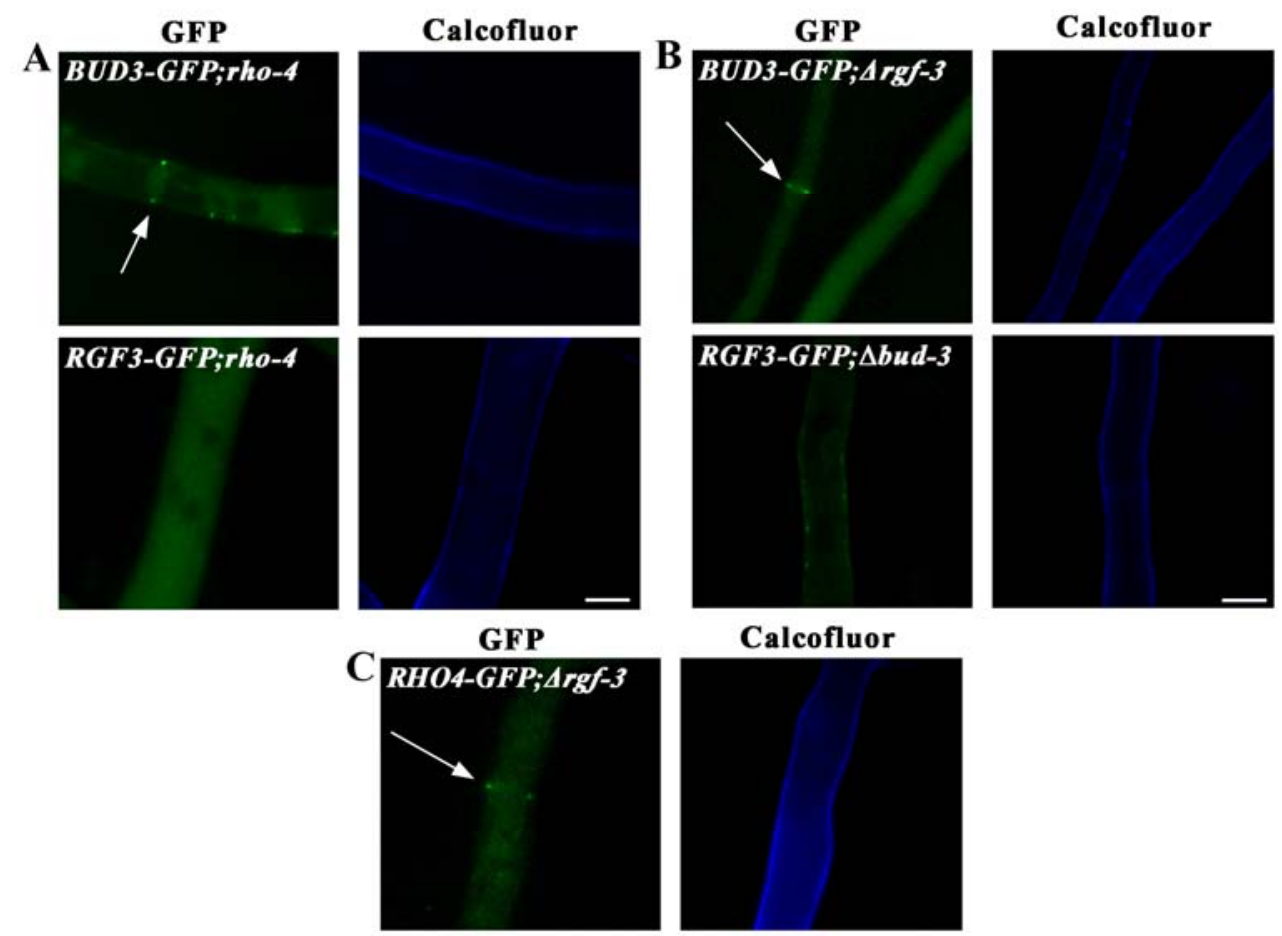

\section{Calcofluor}
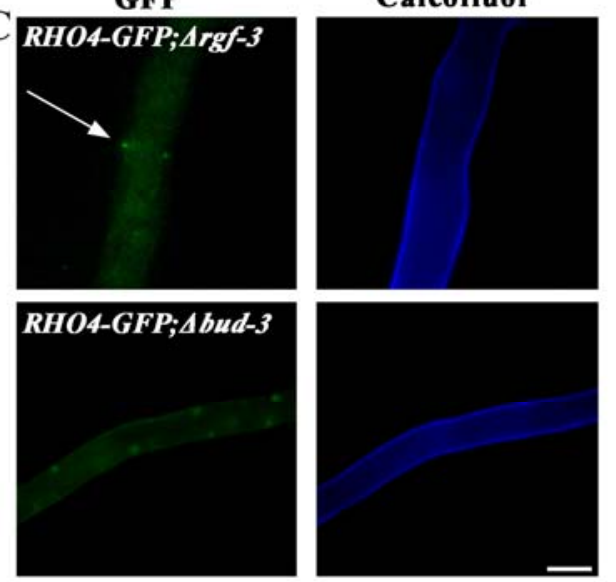

Fig. 13: The localization of RGF3 depends on BUD3 and on RHO4. Distribution of the GEF-GFP fusion proteins in $\Delta r h o-4$ (A) and the respective other $\Delta g e f$ mutant (B). BUD3-GFP forms cortical rings in both aseptate strains (A and $\mathbf{B}$, arrows in upper panels), while RGF3-GFP is distributed in a diffuse manner in the two deletion backgrounds (A and B, lower panels). (C) GFP-RHO4 forms cortical rings in $\Delta r g f-3$ (upper panel; arrow) and localizes in a patchy manner in $\Delta b u d-3$ (lower panel). The cell wall is stained with Calcofluor White. Bars $=5$ $\mu \mathrm{m}$. 
Distinct localizations of both GEFs were observed (Fig. 13A), suggesting independent functions of the two GEFs and the presence of two distinct RHO4 modules. While BUD3GFP localized as cortical dots and rarely as cortical rings in rho-4(RIP) (three cortical rings observed per 27 visual fields; Table 5), potentially marking future sites of septation, RGF3GFP was distributed diffusely throughout the cytoplasm. When the distribution of the two GEFs in the respective other gef mutant background was determined to look for localization dependencies between the two GEFs, a similar pattern was found (Fig. 13B). BUD3-GFP localized as cortical rings in $\Delta r g f-3$ (31 cortical rings per 22 visual fields; Table 5). At three of these 31 cortical rings, we observed accumulation of chitin, suggesting initial attempts to generate a septum. In contrast, RGF3-GFP localized in a diffuse manner throughout the cytoplasm of $\Delta b u d-3$. Thus, cortical BUD3 rings can be formed in strains lacking RHO4 and RGF3, while RGF3 is dependent on RHO4 and BUD3 for cortical localization. However, the number of observed cortical BUD3-GFP rings was much lower in rho-4(RIP) than in $\Delta r g f-3$ (three rings per 27 visual fields versus 31 rings per 22 visual fields, respectively; Table 5), suggesting that RHO4 may be involved in reinforcing the localization of BUD3 and in the stabilization of a hypothetical marker complex at the cortex.

Table 5: Summary of the localization patterns observed for BUD4, BUD3, RHO4 and RGF3

\begin{tabular}{|c|c|c|c|c|}
\hline & RHO4-GFP & RGF3-GFP & BUD3-GFP & BUD4-GFP \\
\hline \multirow[t]{2}{*}{ rho-4(RIP) } & \multirow{2}{*}{$\begin{array}{l}\text { constricting ring; } \\
\text { mature septal pore }\end{array}$} & $0 / 59$ & $3 / 27$ & $0 / 30$ \\
\hline & & cytosolic & cortical dots & cortical dots \\
\hline \multirow[t]{2}{*}{$\Delta r g f-3$} & $5 / 9$ & \multirow{2}{*}{ constricting ring } & $31 / 22$ & 45 / 23 \\
\hline & cortical rings & & cortical rings & cortical rings \\
\hline \multirow[t]{2}{*}{$\Delta b u d-3$} & $0 / 15$ & $0 / 10$ & \multirow{2}{*}{$\begin{array}{l}\text { constricting ring; } \\
\text { mature septal pore }\end{array}$} & $36 / 30$ \\
\hline & large patches & cytosolic & & cortical rings \\
\hline \multirow[t]{2}{*}{$\Delta b u d-4$} & $0 / 39$ & $0 / 28$ & not detected & \multirow{2}{*}{$\begin{array}{l}\text { constricting ring; } \\
\text { mature septal pore }\end{array}$} \\
\hline & large patches & large patches & cytosolic? & \\
\hline
\end{tabular}

cortical rings per visual fields

prominent localization

localization in complemented mutant

This was consistent with the observation that GFP-RHO4 localized as cortical rings in $\Delta r g f-3$ (five cortical rings per nine visual fields; four of them showed accumulation of chitin at these sites; Table 5), but in a patchy manner in $\Delta b u d-3$ (Fig. 13C), Thus, RHO4 localization at 
incipient septation sites was facilitated by BUD3, but proper RHO4 localization was independent of RGF3. However, the ability of RHO4 to associate with the cortex (although in large patches and not distinct dots) was not affected by deleting bud-3 (Fig. 13C). These data indicate two independent functions of RHO4 that are regulated via the exchange factors BUD3 and RGF3, respectively. The different localization patterns and dependencies for localization further suggest that the BUD3-RHO4 module is acting prior to the RGF3-RHO4 module.

Dominant-active RHO4 was expressed in both GEF deletion strains looking for a GEFindependent septum formation. The expression of daRHO4 was verified by Western blot experiments (Fig. 14A). The artificial activation of RHO4 suppressed the septation defect of $\Delta b u d-3$ (Fig. 14B) but not the slow-growth phenotype of $\Delta b u d-3$ (15\% of wild-type growth). In $\Delta r g f-3$ however, no septa were induced by introducing dominant-active RHO4 (Fig. 14B) implying further functions of RGF3 during septation others than being a RHO4-GEF.

$\mathbf{A}$

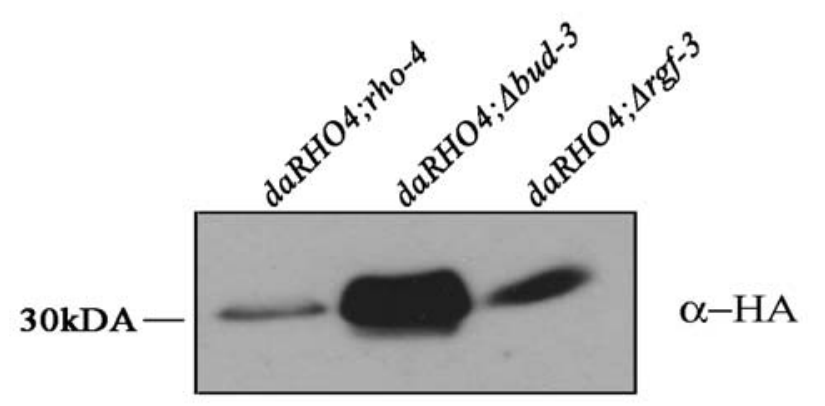

B

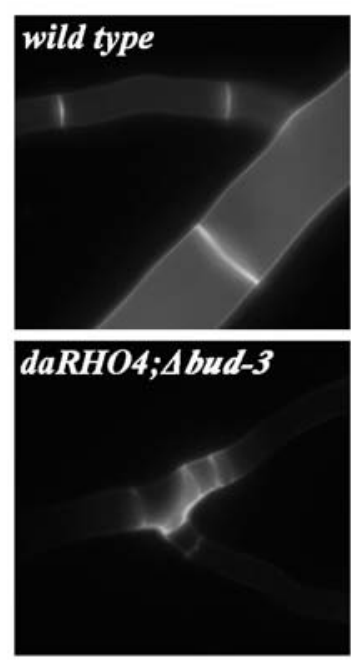

daRHO4;rho-4

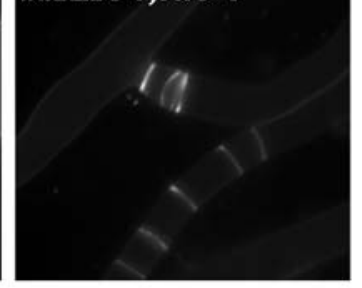

daRHO4;4rgf-3

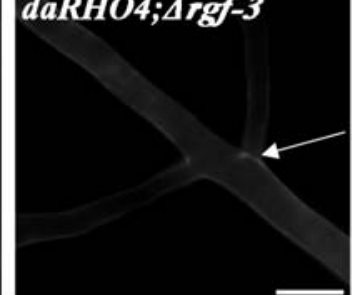

Fig. 14: The septation defect of $\Delta b u d-3$, but not of $\Delta r g f-3$ is suppressed by dominant-active (da)RHO4. (A) Expression of daRHO4 was verified by Western blot analysis using anti-HA antibodies. (B) Ectopic expression of daRHO4 leads to the formation of abnormal and irregular distributed septa in $\Delta b u d-3$, whereas no septa are induced in $\Delta r g f-3$. Note that the arrow does not mark a septum at the base of the site branch, but the Calcofluor White stained cell wall of the leading hypha proceeding above. Cell wall and septa are stained with Calcofluor White. $\mathrm{Bar}=10 \mu \mathrm{m}$.

Because of the presence of a well-conserved PH and CNH domain in RGF3 that could mediate interactions with further proteins, it was examined which region of RGF3 was responsible for proper localization. RGF3_GEF_PH-GFP (amino acids 1-1270) and RGF3_PH_CNH-GFP (amino acids 1077-1688) were expressed in wild-type background (Fig. 15A) and both constructs were tested for their ability to localize in a proper manner during septum formation. A protein harbouring only the $\mathrm{CNH}$ domain (amino acids 1323- 
1688) was unstable and not detectable in Western blot experiments. The two mutant proteins RGF3_GEF_PH-GFP and RGF3_PH_CNH-GFP localized primarily in the cytosol and no constricting rings during septum formation could be detected. However, a faint accumulation of both proteins at mature septa was observed (Fig. 15B). Thus, the presence of the GEF as well as the CNH domain is necessary for proper localization of RGF3 to the forming septum.

A

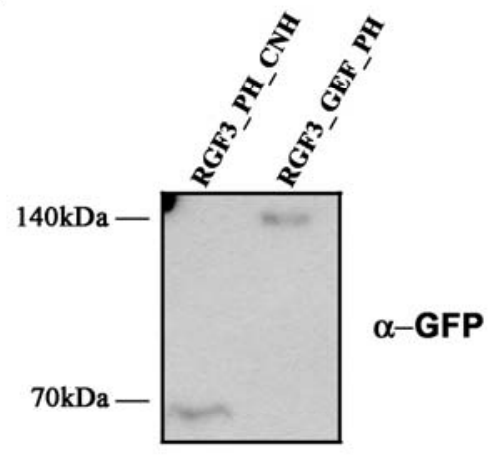

B
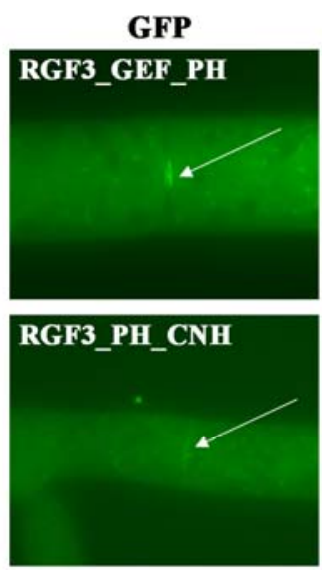

FM4-64
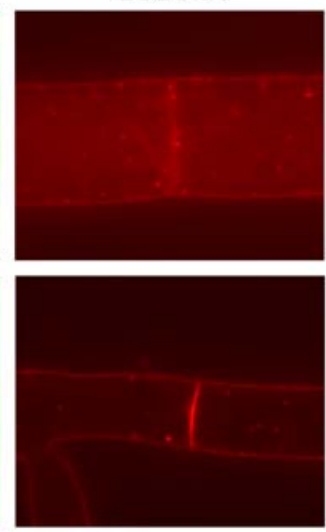
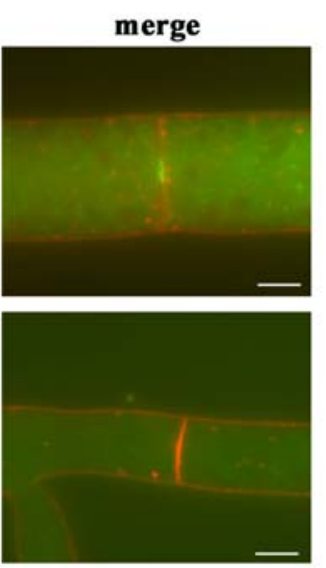

Fig. 15: Truncated RGF3-GFP variants are not able to localize properly. (A) The expression of RGF3_GEF_PH (aa1-1270) containing the GEF and the PH domain of RGF3 as well as RGF3_PH_CNH (aa1077-1688) containing the $\mathrm{PH}$ and the $\mathrm{CNH}$ domain of RGF3 was verified in Western blot experiments using anti-GFP antibodies. (B) Both RGF3 constructs are not associated with the contracting septum. In the RGF3_GEF_PH and RGF3_PH_CNH strain only $20 \%$ and $10 \%(\mathrm{n}=10)$ of observed mature septa, respectively, display a weak GFP signal that is indicated by the arrows. The plasma membrane and endomembranes are stained with FM4-64. Bars $=5 \mu \mathrm{m}$.

To examine if both domains are required for the function of RGF3, RGF3_GEF_PH and RGF3_PH_CNH expressing strains were crossed with $\Delta r g f-3$. Preliminary data suggest that both truncated RGF3 variants are unable to complement the deletion phenotype. However, further work is needed to confirm that the GEF and the CNH domains are necessary for full RGF3 function.

\subsection{The two RHO4 modules BUD3-RHO4 and RGF3-RHO4 are acting prior to actomyosin ring formation}

Rasmussen and Glass have shown that rho-4 was unable to form actin rings (Rasmussen \& Glass, 2005). To determine which of the two RHO4 modules is required for actin ring formation, I asked if cortical BNI1 rings are formed in $\Delta r g f-3$ and $\Delta b u d-3$. BNI1 was used as a marker for the formation of actin rings, since in other organism such as $S$. cerevisiae or $A$. nidulans formins are known to be required for actin ring formation during 
cytokinesis/septation (Sharpless \& Harris, 2002, Vallen et al., 2000). First, a bni-1-gfp fusion construct under the control of the ccg-1 promoter targeted to the his-3 locus was generated that complemented the lethality of $\Delta b n i-1$. Expression of BNI1-GFP was verified by Western blot experiments (Fig. 11). Similar to the situation in A. nidulans (Sharpless \& Harris, 2002), BNI1-GFP localized in a dynamic fashion to the forming septum and to the growing hyphal tips of N. crassa (Fig. 16A; Fig. 19C). Consistent with a function of the contractile actomyosin ring during the actual septation process, we observed BNI1-GFP accumulating as constricting ring at the onset of septum constriction that disappeared from the mature septum. An exemplary TIFF series of time course experiments with BNI1-GFP is shown in Fig. 19C at the end of section 3.6.

$\mathbf{A}$

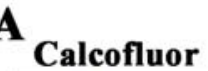

GFP merge BNII-GFP; $\Delta$ bni-I
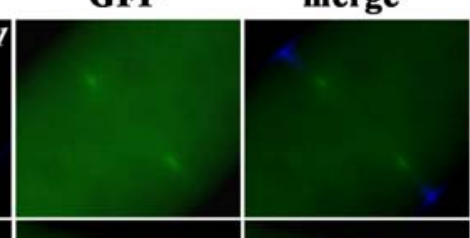

B
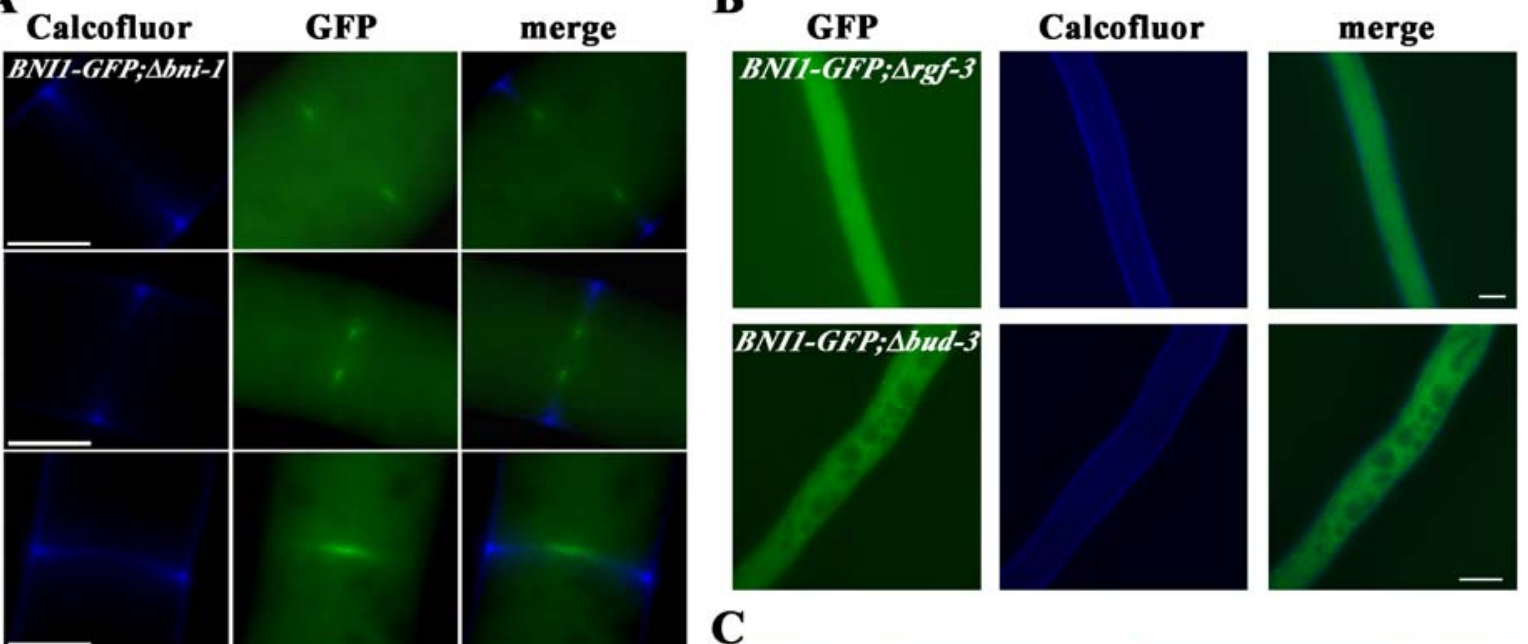

C
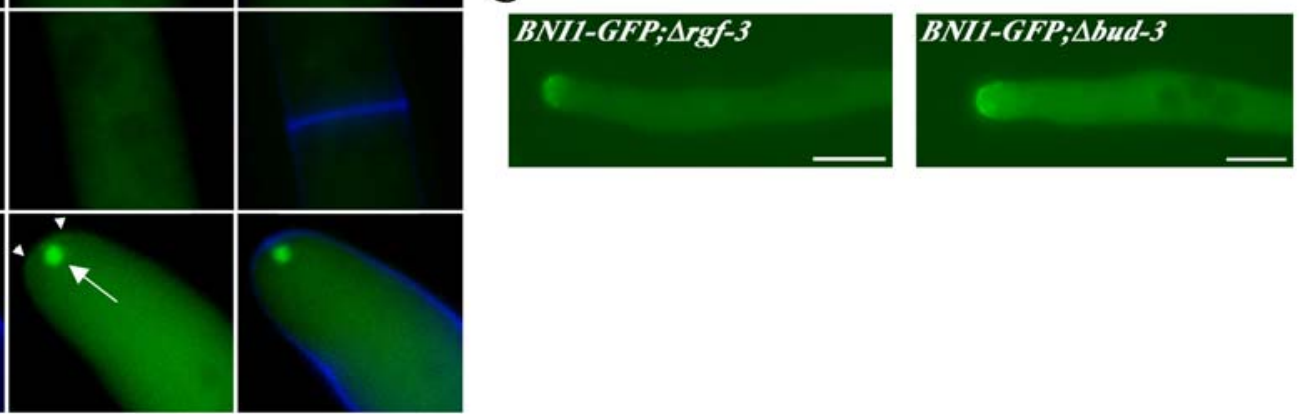

Fig. 16: The formation of cortical BNI1 rings requires the presence of RGF3 and BUD3. (A) Localization of a BNI1-GFP fusion protein in a $\Delta b n i-1$ background. BNI1-GFP accumulates at incipient septation sites and forms constricting rings. Septal pores of mature septa are not labelled. Furthermore, BNI1-GFP localizes to the Spitzenkörper (arrow) and the apical tip (arrow heads) of a growing hypha. A TIFF series of BNI1-GFP localization during septum formation is shown in Fig.19C. (B) In $\Delta r g f-3$ and $\Delta b u d-3$, BNI1-GFP localizes in the cytoplasm and no cortical rings are formed. (C) BNI1-GFP accumulates at the hyphal apex in the two mutant strains. Cell wall and septa are labelled with Calcofluor White. Bars $=5 \mu \mathrm{m}$.

In $\Delta r g f-3$ and $\Delta b u d-3$ cells, no formation of cortical BNI1-GFP rings was observed (Fig. 16B), although BNI1 was still able to accumulate at the hyphal apex in both mutants (Fig. 
16C). However, there were minor differences in the localization pattern of BNI1-GFP at hyphal tips in $\Delta r g f-3$ and $\Delta b u d-3$ compared to the pattern observed in the complemented strain. While BNI1-GFP accumulated in the Spitzenkörper (Spk) and at the apical tip in the complemented strain, no Spk localization of BNI1-GFP was observed in both GEF deletion mutants. BNI1-GFP only localized at the hyphal apex. These localization differences could be a consequence of the slow-growth phenotype of both mutants. The size and stability of the Spk, which is predicted to function as a vesicle supply center and is crucial for hyphal extension (reviewed in Harris et al., 2005), is directly correlated with the growth rate of the fungus (Araujo-Palomares et al., 2007, Riquelme et al., 2000, Riquelme et al., 2002). In slowgrowing mutants the Spk is often less prominent and sometimes even undetectable. This was for example described for the two ropy mutants ro- 1 and $r o-3$, which are deficient in dynein and dynactin (Riquelme et al., 2000).

The result that no BNI1-GFP rings are formed in both GEF deletion mutants indicate that both RHO4 modules are required for formin assembly at septa and thus, probably also for the formation of the actomyosin ring.

\subsection{The localization of BUD4 at the cortex is independent of BUD3 and RGF3}

In the screen for aseptate mutants in the $N$. crassa deletion strain collection I identified in addition to $\Delta b u d-3$ and $\Delta r g f-3$ a strain deleted for the gene NCU00152 (Table 4). This strain displayed typical rho-4-like defects, such as slow growth (ca. 15\% of wild type and similar to $\Delta r h o-4, \Delta r g f-3$ and $\Delta b u d-3)$, abnormal branching, thin hyphae, cytoplasmic leakage and abolished conidiation (Fig. 17A). Sequence analysis revealed significant homologies to anillin-related proteins (expect values of $3.8 \mathrm{e}^{-17}$ and $1.1 \mathrm{e}^{-35}$ to budding yeast Bud4 $\mathrm{p}$ and fission yeast Mid2, respectively), that are known to be involved in cytokinesis/septation. Because the function of Mid2 and its relationship to Mid1 in S. pombe is currently poorly defined (Berlin et al., 2003), the protein NCU00152 with a predicted size of $156 \mathrm{kDa}$ was named BUD4, a name also suggested in a sequence-based annotation review (Walther \& Wendland, 2003). Next, the localization of BUD4 in N. crassa was investigated to analyze if BUD4 also has a function in the septation process. Therefore, a BUD4-GFP fusion construct targeted to the his-3 locus under the control of the $c c g-1$ promoter was generated, which complemented the mutant defects. Expression of BUD4 was weak and detectable in Western blot experiments only after immunoprecipitation of the protein (Fig. 11). It localized as a 
cortical ring prior to septum initiation, in a dynamic fashion to the forming septum and persisted around the mature septal pore (Fig. 17B; Fig. 19E). An exemplary TIFF series of time course experiments with BUD4-GFP is shown in Fig. 19E at the end of section 3.6.

A

wild type
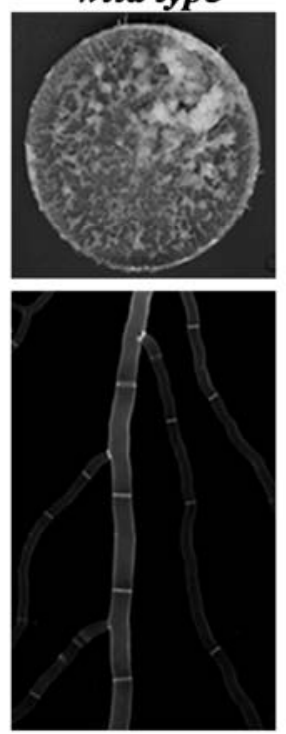

C

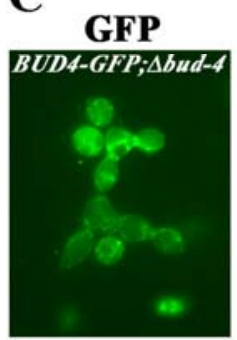

$\Delta b u d-4$
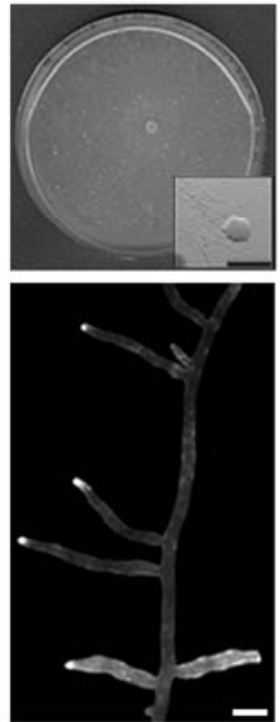

B
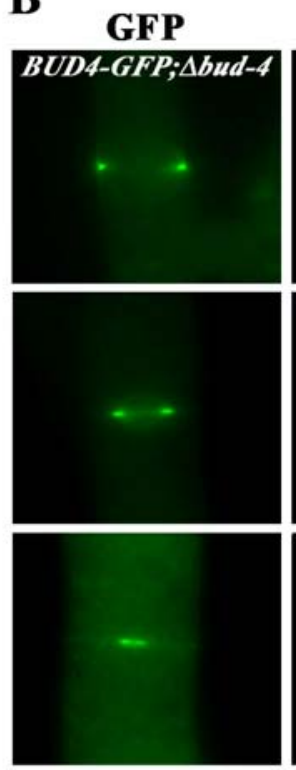

D

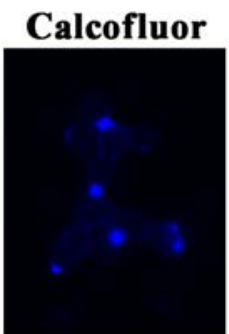

merge

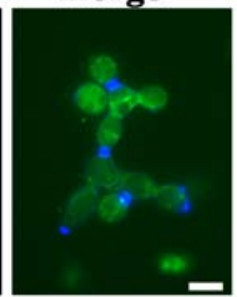

Calcofluor
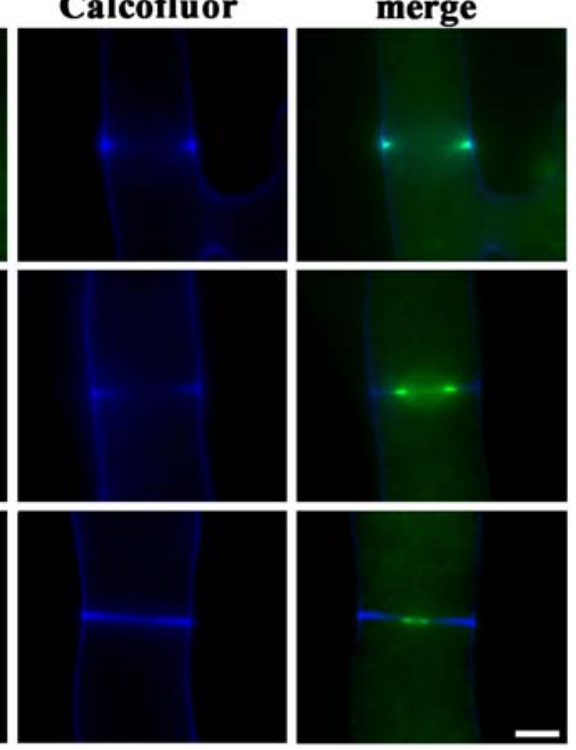
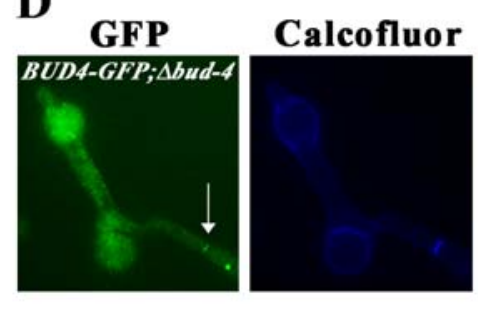

merge

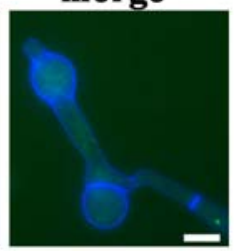

Fig. 17: The anillin-like protein BUD4 is required for septum formation. (A) $\Delta b u d-4$ displays phenotypic characteristics identical to $r h o-4(\mathrm{bar}=10 \mu \mathrm{m})$; leaking cytoplasm is shown as insert (bar $=500 \mu \mathrm{m})$. (B) BUD4GFP localizes at incipient septation sites and the forming septum. Note that BUD4-GFP remains at the septal pore of mature septa. TIFF series of BUD4-GFP localization during septum formation and during unsuccessful septation site selection are shown in Fig. 19E, F. (C, D) Distribution of BUD4-GFP in dormant conidiospores and young germlings. Neither in dormant conidiospores (C) nor in young germlings (D) is BUD4-GFP distributed in a polarized manner. The arrow marks a septum labelled with BUD4-GFP. Cell wall and septa are labelled with Calcofluor White. Bars $=5 \mu \mathrm{m}$.

Interestingly, during the time course experiments with BUD4 the accumulation of BUD4-GFP dots and rings that moved along the cortex, but disappeared without establishing functional septa was observed (in two of 12 monitored septation events). This is consistent with a putative marker function of BUD4 during the selection of the septation site. An exemplary TIFF series of such an unsuccessful septation event is shown in Fig. 19F at the end of section 3.6. Furthermore, we observed no polar localization of BUD4 in dormant conidia, during conidial germination and at the hyphal apex of mature hyphae (Fig. 17C, D), making a prominent function of BUD4 for polar cell growth unlikely. However, the persistence of 
BUD4, BUD3 and RHO4 around the septal pore may suggest additional (marker) functions at the mature septum (Fig. 19E).

To test whether BUD4 is acting prior to the both GEFs RGF3 and BUD3 and to RHO4 as implicated by its localization pattern, BUD4-GFP was expressed in $\Delta b u d-3, \Delta r g f-3$ or $\Delta r h o-4$. In $\Delta b u d-3$ or $\Delta r g f-3$, the fusion protein localized as cortical rings (36 and 45 cortical rings per 30 and 23 visual fields, respectively; Table 5), indicating that its localization was independent of the two GEFs (Fig. 18A). A role of BUD4 upstream of the two RHO4 modules was further supported by the inability of GFP-RHO4 and of RGF3-GFP to form cortical rings in $\Delta b u d-4$ (Fig. 18B; no cortical rings per 39 and 28 visual fields, respectively; Table 5).
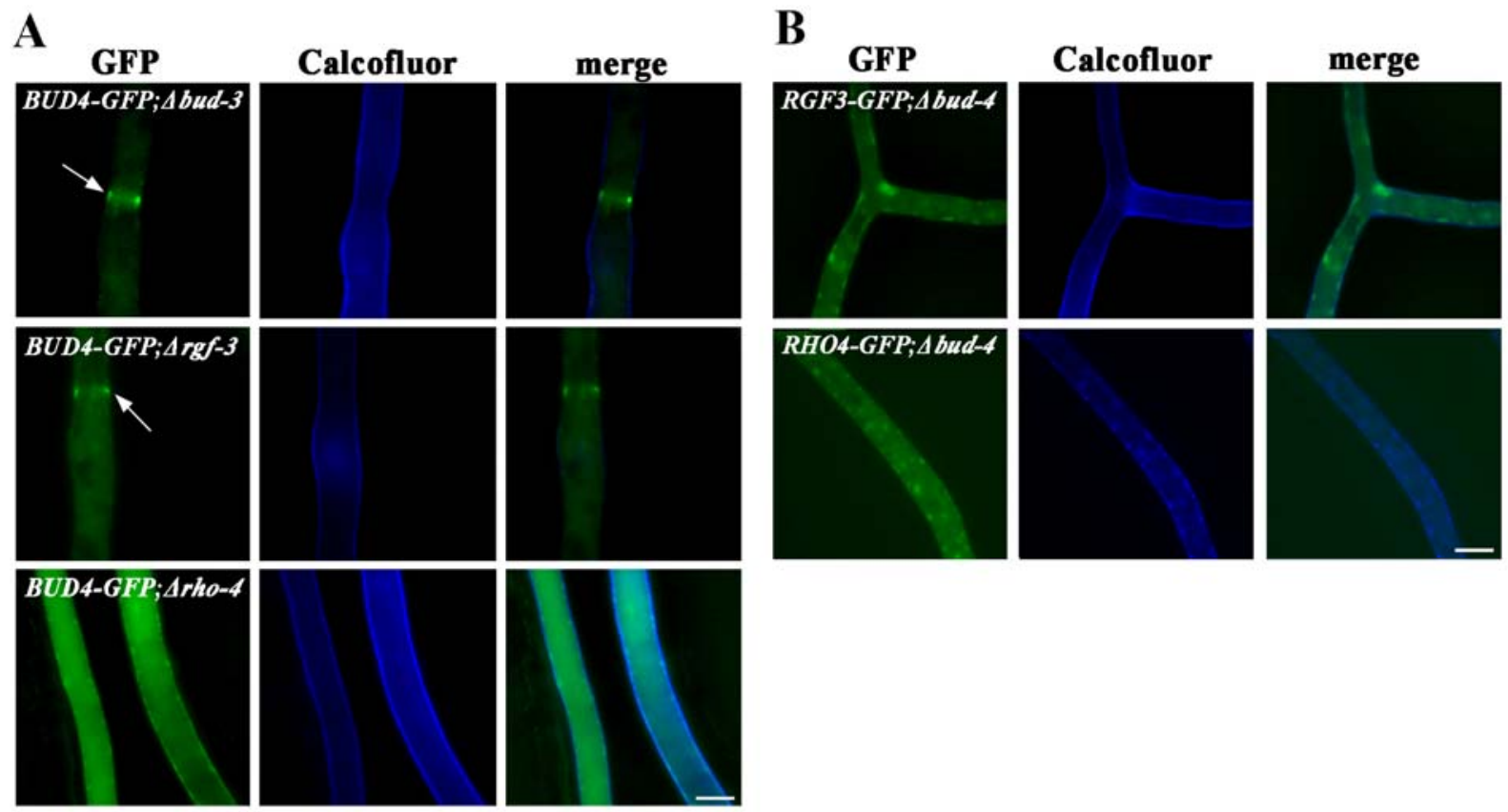

Fig. 18: BUD4 acts as a cortical marker upstream of RHO4. (A) Localization of BUD4-GFP in $\Delta b u d-3, \Delta r g f-3$ and $\Delta r h o-4$. BUD4-GFP localizes as cortical rings in both aseptate GEF deletion strains (upper and middle panels; indicated by arrows). Only localization in cortical dots, but not in proper BUD4-GFP rings is observed in $\Delta r h o-4$ (lower panel). (B) Localization of RGF3-GFP and RHO4-GFP in $\Delta b u d-4$. Both proteins localize in a patchy manner in the aseptate deletion strain. Cell wall and septa are labelled with Calcofluor White. Bars $=5 \mu \mathrm{m}$.

Unfortunately, we were unable to detect BUD3-GFP in $\Delta b u d-4$ by live microscopy, presumably because its cortical localization was abolished and its cytosolic signal was too weak to be detected. Interestingly, RHO4-GFP localized in a patchy manner in $\Delta b u d-4$ that frequently correlated with aberrant accumulation of cell wall material along the cortex. This was also observed for RHO4-GFP in the $\Delta b u d-3$ mutant and could be indicative of uncoordinated attempts of septum formation in mutants with abolished site selection capacity. However, we also found no cortical BUD4-GFP rings in $\Delta r h o-4$ (no cortical rings observed in 30 visual fields; Table 5), but the fusion protein accumulated in cortical dots in a manner that 
was highly similar to BUD3-GFP in $\Delta r h o-4$ (Fig. 13A). This may indicate that BUD4 is able to accumulate at the cortex independently of BUD3 and of RHO4, but BUD3-dependent RHO4 activity is needed for the correct organization of BUD4 in a ring prior to septation.

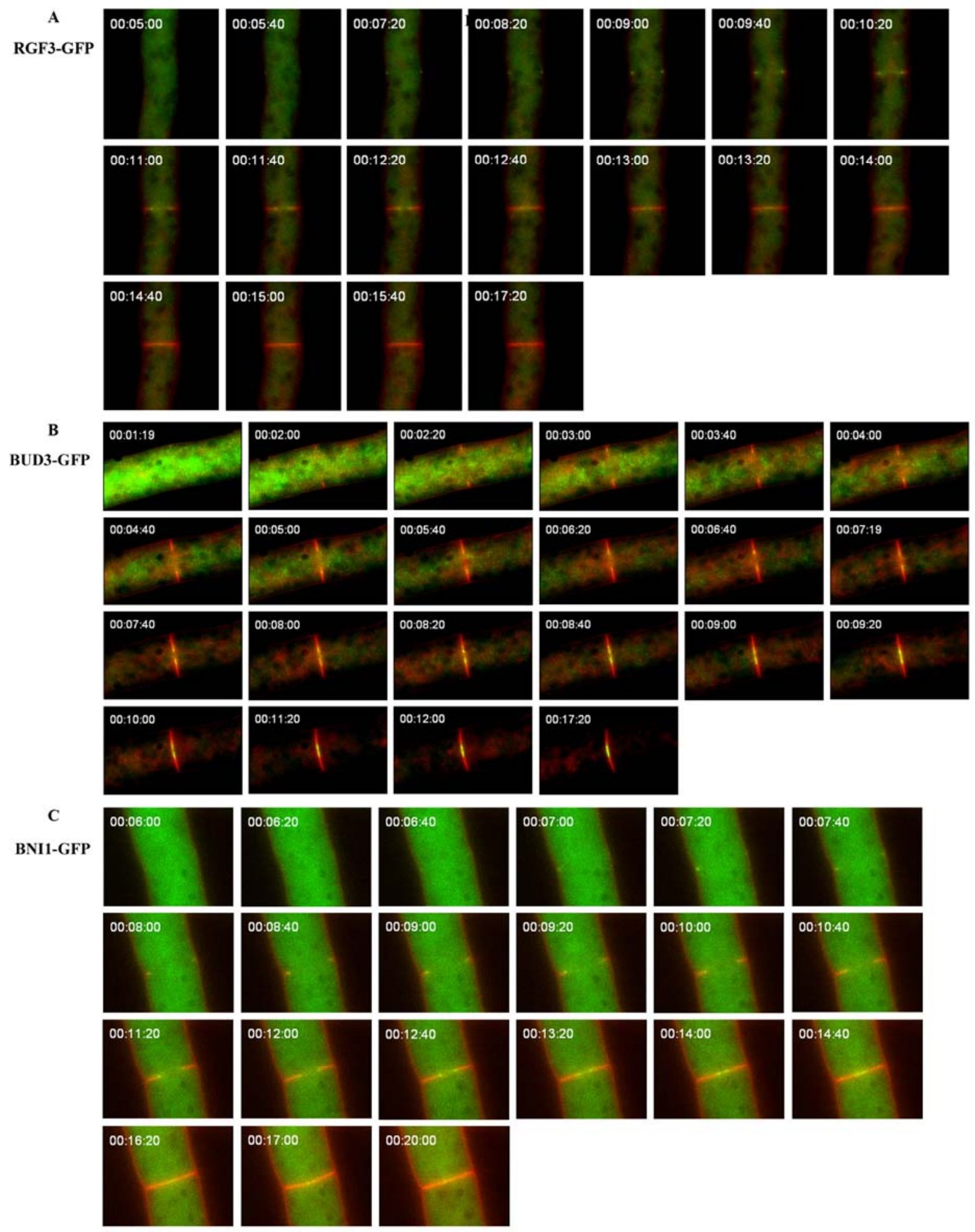

Fig. 19: TIFF series of RGF3-GFP (A), BUD3-GFP (B) and BNI1-GFP (C) localization during septum formation. Note that BUD3-GFP, but not RGF3-GFP and BNI-GFP remain at the septal pore of the mature septum. The plasma membrane and endomembranes are stained with FM4-64. Time is given in hrs:min:sec. 


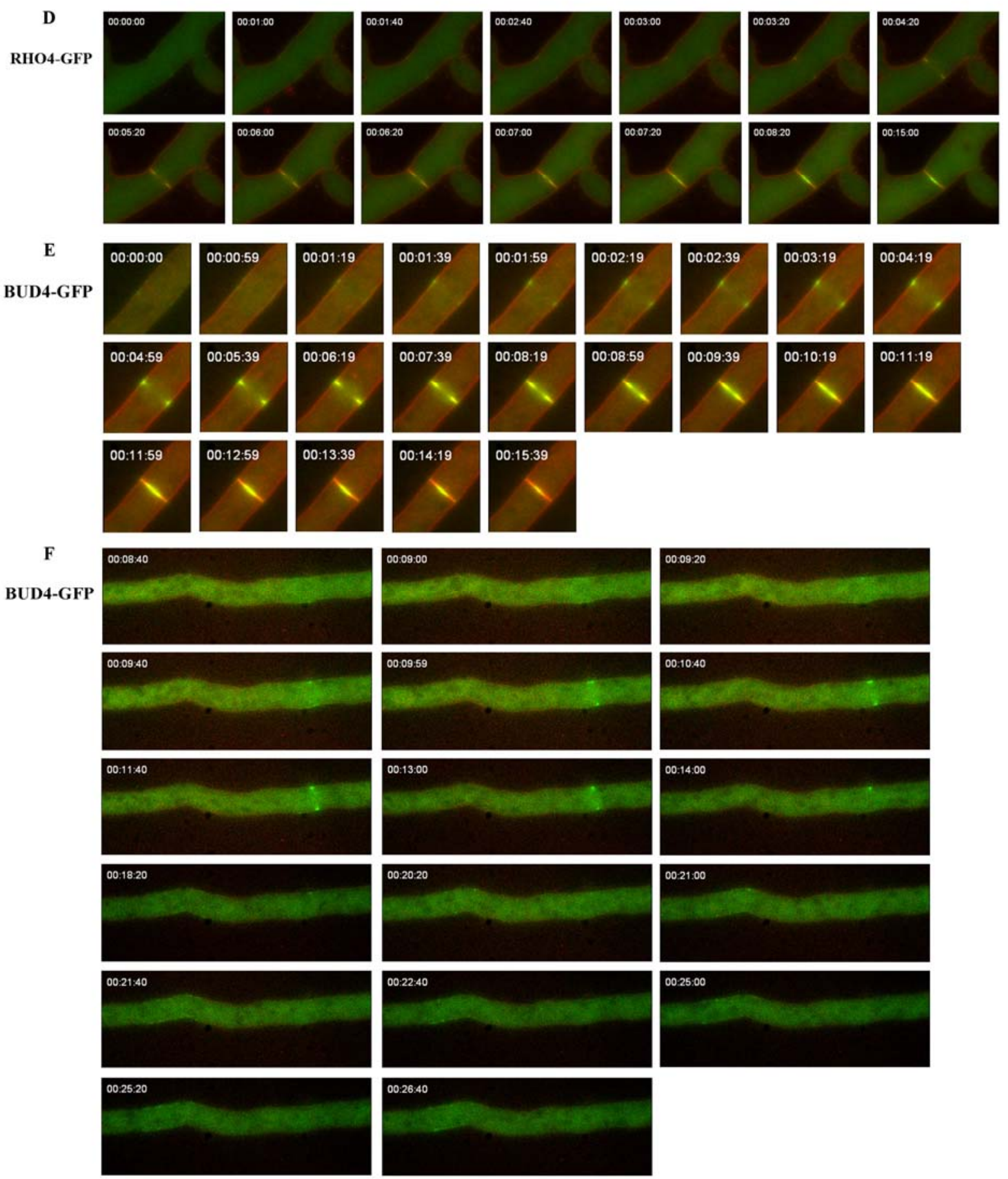

Fig. 19 (continued): TIFF series of RHO4-GFP (D) and BUD4-GFP (E) localization during septum formation and BUD4-GFP (F) localization during a failed attempt of septum formation. Note that RHO4GFP and BUD4-GFP remain at the septal pore of the mature septum. The plasma membrane and endomembranes are stained with FM4-64. Time is given in hrs:min:sec. 


\subsection{CDC12-GFP and CLA4-GFP localize as constricting rings at forming septa}

Septins and PAK kinases are described to be involved in cytokinesis/septation (reviewed in (Balasubramanian et al., 2004, Gonzalez-Novo et al., 2009b, Walther \& Wendland, 2003). Thus, the N. crassa genome was searched for homologues of genes of both families. Amongst others, the two genes NCU00406 and NCU03795 were identified, which were named according to their homologues in S. cerevisiae CLA4 and CDC12, respectively. To investigate if these two proteins also have a function during the formation of septa, CLA4and CDC12-GFP fusion constructs were generated and targeted to the his-3 locus under the control of the $c c g-1$ promoter in wild type background. The protein level of CDC12 was detectable in cell extracts by Western blot experiments, whereas CLA4 expression could only be detected by Western blot analysis after immunoprecipitation (Fig. 20A). Interestingly, CLA4 showed a double band on the immunoblot with the upper band representing the fulllength CLA4 and a protein of smaller size, representing a degraded or modified product.

A

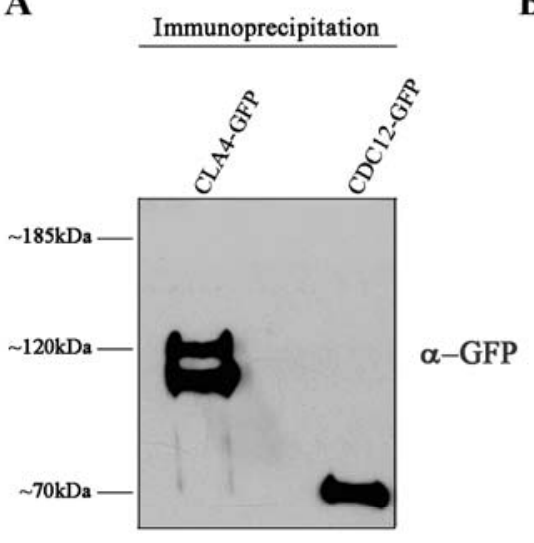

B

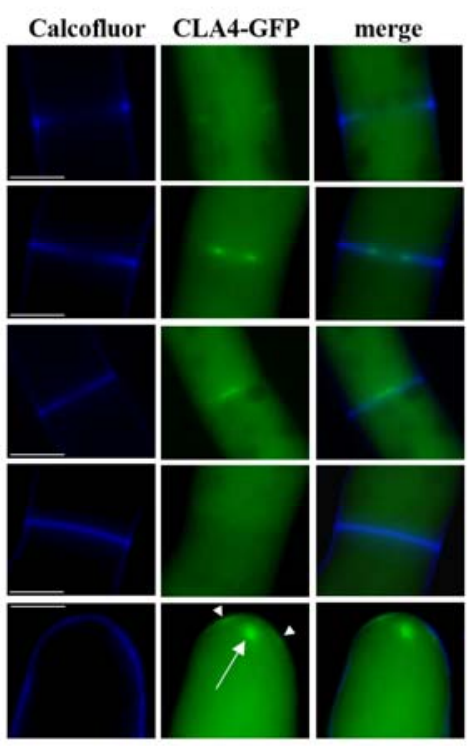

C

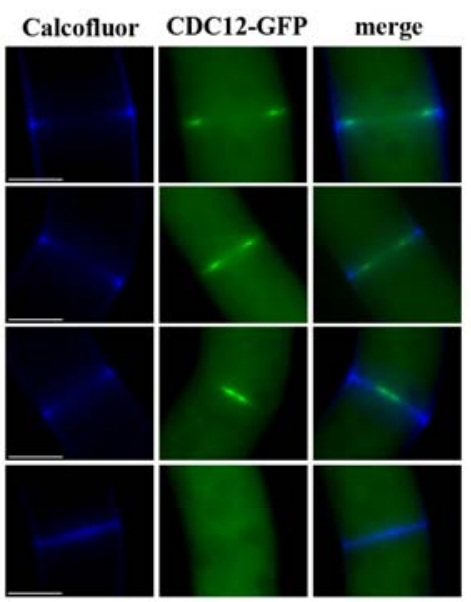

Fig. 20: CDC12-GFP and CLA4-GFP localize as constricting rings to forming septa. (A) Expression of GFP fusion proteins of $\mathrm{CDC} 12(70 \mathrm{kDa})$ and CLA4 $(120 \mathrm{kDa})$ in wild-type background are verified by immunoprecipitation with anti-GFP antibodies and subsequently Western blot analysis using anti-GFP antibodies. (B) CLA4-GFP and (C) CDC12-GFP accumulate at incipient septation sites and form constricting rings. Septal pores of mature septa are not labelled. Furthermore, CLA4-GFP localizes to the apical tip (arrow heads) and to the Spitzenkörper (arrow) of growing hyphae (B, lowest panel). Cell wall and septa are labelled with Calcofluor White. Bars $=5 \mu \mathrm{m}$. 
CDC12 and CLA4 localized to the forming septum as a contractile ring. Both proteins did not persist around the septal pore after the septum was completed (Fig. 20B,C). Furthermore, CLA4-GFP also accumulated as an apical cap at the hyphal tip and in the Spitzenkörper of growing hyphae (Fig. 20B lowest panel) indicating an additional role during polar growth. Since the functionality of both fusion proteins could not be confirmed so far (CDC12-GFP and CLA4-GFP did not suppress the mutant phenotype of the respective deletion strain), the work with these two proteins was not continued. 


\section{Discussion}

\subsection{The cell division machinery is conserved in $N$. crassa}

The annotation of multiple fungal genomes and subsequent phylogenomic and proteomic approaches have revealed that the cell division machinery for septation is largely conserved across the fungal kingdom (Banuett et al., 2008, Borkovich et al., 2004, Eme et al., 2009, Garcia-Pedrajas et al., 2008, Harris et al., 2009). On the basis of sequence similarities to proteins involved in bud-site selection and septation in other fungi, I identified and characterized proteins in this work that are required for selection of the septation site and septum formation in the filamentous fungus $N$. crassa. However, functional differences seem to exist between unicellular yeasts, filamentous hemiascomycetes and filamentous euascomycetes, which may be explained by different morphologies and phylogenetic relationships. These common mechanisms and species-specific differences will be discussed in the following paragraphs.

\subsection{The two RHO4 modules BUD3-RHO4 and RGF3-RHO4 are involved in septation}

Rho GTPases function as key regulators of the actin cytoskeleton and the regulation of cell cycle progression. Consistently, fungal Rho proteins are also involved in the septation process (Park \& Bi, 2007). In the euascomycetes $N$. crassa and A. nidulans the Rho GTPase RHO4/AnRho4 is essential for septation and normal hyphal morphology (Park \& Bi, 2007, Rasmussen \& Glass, 2005, Rasmussen \& Glass, 2007, Si et al., 2010). In this work, BUD3 was identified as component of the septation network and as activator of RHO4 (Justa-Schuch et al., 2010). BUD3 specifically stimulated the in vitro guanine nucleotide exchange activity of RHO4, and its deletion led to mutant characteristics that were identical to those described for $\Delta r h o-4$ (Rasmussen \& Glass, 2005). In addition, BUD3 localized as RHO4 (Rasmussen \& Glass, 2007) to future septation sites, forming septa and around the mature septal pore. Consistent with this, the $A$. nidulans BUD3 homologue AnBud3 also acted as activator of the RHO4 homologue AnRho4 as well as of N. crassa RHO4 in vitro. Furthermore, the absence of BUD3 or RHO4 in both organisms resulted in aseptate strains, and both proteins localized to septa (Justa-Schuch et al., 2010, Si et al., 2010). Thus, the BUD3-RHO4 module and its function during septation seem to be conserved in these two fungal species of the euascomycetes. 
Interestingly, RHO4 in N. crassa was also activated in in vitro experiments through a second GEF, which is highly homologous to fission yeast Rgf3p. Like BUD3 and RHO4 RGF3 localized to future septation sites and formed a constricting ring there. Since N. crassa $\Delta r g f-3$ also shared an identical phenotype with $\Delta r h o-4$, I conclude that RHO4 has two distinct functions during septation that are specifically regulated through one of the two GEFs. A RGF3 homologue is also present in A. nidulans encoded by the gene AN7783 (expect value of 0.0). Whether this homologue functions as an AnRho4 GEF and is involved in septation in $A$. nidulans will be investigated in future.

\subsection{BUD3 functions in site selection and the actual septation process}

Bud3p has originally been identified as marker protein to determine the axial budding pattern in haploid S. cerevisiae cells (Chant \& Herskowitz, 1991). Since mutants lacking BUD3 also displayed a mild cytokinesis defect, and Bud3p targets the cyclin Clb2 to the bud neck, it may also have additional functions during cytokinesis in budding yeast (Bailly et al., 2003, Chant et al., 1995, Roemer et al., 1996). The landmark function of Bud3p for the selection of septation sites is conserved in A. gossypii, where it is involved in the proper positioning of the contractile ring (Kaufmann \& Philippsen, 2009, Wendland, 2003). Bud3p localizes to sites of septation, but not to the hyphal tips in Ashbya or growing buds in budding yeast. A similar localization pattern was observed for BUD3 in N. crassa (Justa-Schuch et al., 2010) and AnBud3 (Si et al., 2010), arguing for a general function of Bud3 as a common marker for selecting future septation sites in unicellular and filamentous hemi- and euascomycetes. This is further supported by the recent finding that the $N$. crassa BUD3 homologue RhoGEF Gef2p in $S$. pombe localizes in interphase nodes around the equator of interphase cells along with the anillin-like protein Mid1p, which provides the spatial cue for cell division in $S$. pombe (Moseley et al., 2009).

In addition to its marker function, BUD3 seems to have a prominent role in the actual septation process in euascomycetes. While Bud3p is nonessential for bud formation and septation in the hemiascomycetes $S$. cerevisiae and A. gossypii, Bud3 homologues in the euascomycetes $N$. crassa and A. nidulans are absolutely required for septum formation. The fact that bud-3 deletion in S. cerevisiae and A. gossypii only leads to an altered budding pattern or to delocalized septa, respectively, but results in aseptate hyphae in N. crassa and A. nidulans, coincides with the observation that Rho4 has different functions in these organisms. While N. crassa RHO4 and AnRho4 are essential for septation, no function of Rho4p during 
cytokinesis is described in budding yeast. Here, Rholp is a key regulator of cytokinesis (Park \& Bi, 2007). Consistently, Rom2p, the homologue of the second RHO4-specific GEF RGF3 identified in N. crassa, is not Rho4p- but Rho1p-specific in S. cerevisiae (Ozaki et al., 1996, Park \& Bi, 2007). Thus, the function of BUD3 as a landmark is conserved in hemi- and euascomycetes, but hemiascomycetes may have lost the GEF activity of Bud3p, which is required for the activation of RHO4. These functional differences may be reflected by the distinct localization patterns of these proteins in the different organisms: in N. crassa and A. nidulans the formation of one constricting BUD3 ring was observed, whereas in S. cerevisiae and $A$. gossypii the cortical Bud3p ring did not constrict, but split into two cortical rings embracing the forming septum (Chant et al., 1995, Kaufmann \& Philippsen, 2009, Wendland, 2003).

\subsection{The anillin-related protein BUD4 marks future septation sites}

Furthermore, the anillin-like protein BUD4 was identified as required for septation in $N$. crassa. It shows homology to the landmark protein Bud4p in S. cerevisiae as well as to the anillin-related proteins Mid1p/Mid2p in S. pombe. The two homologues Bud4p and Mid1p are critical for septation site selection in budding and fission yeast, respectively. However, in contrast to their counterpart BUD4 in Neurospora they are not essential for septum formation in the corresponding yeast. S. pombe cells lacking functional Mid1p position their septa randomly (Sohrmann et al., 1996) and BUD4 deletion in haploid S. cerevisiae cells results in an altered budding pattern (cells exhibit a bipolar pattern instead of the axial one) (Chant \& Herskowitz, 1991, Fujita et al., 1994). In S. cerevisiae, Bud4p functionally interacts with Bud3p, and both proteins cooperate as landmark for bud site selection (Chant \& Herskowitz, 1991, Gladfelter et al., 2005, Lord et al., 2002, Sanders \& Herskowitz, 1996). Mid1p in fission yeast provides the spatial cue for cytokinesis by defining the site for recruitment of actomyosin ring components (Paoletti \& Chang, 2000). It is one of the earliest proteins recruited to the future division site assembling in interphase nodes around the equator of interphase cells (Wu et al., 2003). Recently, the N. crassa BUD3 homologue RhoGEF Gef2p in S. pombe was shown to localize along with Mid1p in these nodes (Moseley et al., 2009) suggesting that both proteins may cooperate in providing the spatial cue. The homology of BUD4 to these division site markers argues for a function of BUD4 in determining the septation site in $N$. crassa. This hypothesis is supported by its localization pattern. As described for BUD3, BUD4 marked future septation sites, and localized to the forming 
septum and around the septal pore of mature septa. Furthermore, BUD4-GFP spots that transiently assembled at the cortex, but disappeared without subsequent septum formation were observed, suggesting failed attempts of septum initiation. This was not observed for the proteins BUD3, RGF3 and RHO4, suggesting BUD4 as the earliest marker for septation site selection. This conclusion is further supported by the fact that BUD4 localization as cortical ring is independent of BUD3 and RGF3. Since BUD3 is also suggested to be involved in site selection and since its localization was shown to be dependent on BUD4, both proteins may cooperate as landmark complex determining the future septation site as also described for Bud3p and Bud4p in budding yeast (Chant \& Herskowitz, 1991, Gladfelter et al., 2005, Lord et al., 2002, Sanders \& Herskowitz, 1996). In this context, it will be interesting to investigate by co-immunoprecipitation experiments whether BUD3 and BUD4 physically interact with each other. Interestingly, the presence of a GTP binding motif was described for Bud $4 p$ (Sanders \& Herskowitz, 1996) that is also (poorly) conserved in N. crassa BUD4. Thus, BUD4 may assist the BUD3-RHO4 complex in supplying high levels of GTP for the GTPase in N. crassa. Strikingly, also during animal cytokinesis, Anillin is one of the first proteins recruited to the cleavage site and is thought to play a key role in the proper assembly of the contractile actomyosin ring. It interacts with the major structural components of the ring, such as actin, myosin II and septins, thereby providing a scaffold for ring organization.

Furthermore, Anillin links these ring components to signalling proteins that control cytokinesis (reviewed in D'Avino, 2009). For example, Anillin was shown to interact with the microtubule-bound RacGAP50C in D. melanogaster, which in turn, can interact with and thereby activate the RhoA GEF Pbl (D'Avino et al., 2008, Gregory et al., 2008). In humans Anillin binds to the Rho GTPase RhoA, the key regulator of cytokinesis in humans (Piekny \& Glotzer, 2008).

\subsection{BUD3 and BUD4 do not seem to have a prominent role in polar growth}

In addition to the function of BUD3 and BUD4 in regulating septation initiation, both BUD proteins may also have marker functions during branch initiation or at the hyphal tip. However, deletion of these genes did not abolish polar growth and GFP fusion proteins do not localize at the hyphal tip or emerging branches. Consistently, the BUD3 homologue AnBud3 is also not necessary for polar growth in A. nidulans (Si et al., 2010). Furthermore, similar observations were made in budding and fission yeast and in A. gossypii. In these organisms, 
polar growth was not abolished upon deletion of the respective BUD counterparts and the corresponding GFP fusion proteins did not localize to growing buds, cell ends or hyphal tips (Casamayor \& Snyder, 2002, Sohrmann et al., 1996, Wendland, 2003). Based on these data, I conclude that BUD3 and BUD4 do not have a prominent function for polar growth. However, a minor involvement of the BUD proteins in regulating cell polarity cannot be excluded. The thin and slow-growing hyphae observed in the mutants may be a secondary consequence of lacking septa, a phenotype also observed in mutants of SIN kinase network components that have functions unrelated to Rho GTPase signalling during septation (Dvash et al., 2009, Maerz et al., 2009).

Interestingly, BUD3 as well as BUD4 (and RHO4 (Rasmussen \& Glass, 2007)) remained at the septal pore after septum completion. This is distinct from the situation observed in S. cerevisiae and $A$. gossypii and suggests additional functions of these proteins at the septum in N. crassa and potentially other euascomycetes. Maybe these proteins serve as a marker for the ends of the hyphal compartment. In fission yeast, for example, the two ends of the cell are marked by the cell end markers Tea1p, Tea4p, and Pom1p. Their accumulation to both ends prevents cytokinesis there (Huang et al., 2007). Moreover, they are also involved in growth regulation and microtubular dynamics at the cell ends (Niccoli et al., 2003). A further possible function of BUD3, BUD4 and RHO4 at the mature septum could be to act as a scaffold for organelles, such as the Woronin bodies, which are associated with septa and plug the septal pore upon hyphal injury (Jedd \& Chua, 2000), or for proteins, such as for components of microtubule organizing centres (MTOCs). The latter were shown to be present at septa in A. nidulans (Veith et al., 2005). It will be interesting to explore the septationindependent functions of this BUD4/BUD3/RHO4 landmark complex at the mature septa in future.

\subsection{Expression of dominant-active RHO4 suppressed the septation defect of $\Delta b u d-3$ but not of $\Delta r g f-3$}

The septation defect of $\Delta b u d-3$ can be overcome by dominant-active (da) RHO4, which is consistent with a function of BUD3 upstream of RHO4. In contrast, $\Delta r g f-3$ defects were not suppressed by over-expressing daRHO4. This on the first view confusing observation may be explained by a function of RHO4 as part of the BUD3-BUD4 landmark complex, a notion that is also supported by the necessity of RHO4 for solid localization of BUD3 and BUD4. Artificial activation of RHO4 may overcome the marker function of BUD3-BUD4 (consistent 
with the irregular placement of septa), but the actual septation process may require consecutive cycles of RHO4 activation through the second GEF RGF3. Although originally described as simple "on - off" switches, there is accumulating evidence that the function of small G-proteins requires not only the coordination of the "on" switch, but also that the shuttling between both the active and inactive states of the GTPase is essential for its full signalling (Barale et al., 2006, Bement et al., 2006, D'Avino \& Glover, 2009, Vanni et al., 2005). Alternatively, proper localization and function of RGF3 may be the prerequisite for septation by serving as interaction platform for RHO4 and its effectors, and thus $\Delta r g f-3$ cannot be suppressed by daRHO4. In the literature, there are examples of GEFs that directly bind to GTPase effectors or to scaffolds that form a complex with GTPase effectors, thereby specifying downstream signalling of RHO proteins (reviewed in Buchsbaum, 2007). In this context, a RGF3 construct with the putative Rho interaction helix deleted was generated to investigate if the concerted expression of this catalytic inactive GEF version and daRHO4 can overcome the septation defect in $\Delta r g f-3$. Unfortunately, the corresponding protein was unstable and not detectable in Western experiments. Since in addition to the GEF domain the well-conserved $\mathrm{PH}$ and $\mathrm{CNH}$ domains, which both may mediate protein and/or lipid interactions, are present in RGF3, I investigated, which region of RGF3 is responsible for its proper localization. In this test the GEF as well as the $\mathrm{CNH}$ domain were shown to be necessary to localize properly. Moreover, preliminary data imply that both RGF3 domains are required for its function supporting the idea that the spatial coordination of GEFs is important for their function. Interestingly, RGF3 was found to be expressed as (at least) two isoforms, which only differ in the PH domain. This may be an indication for multiple functions of this protein in fine tuning the septation process.

\subsection{BUD3-RHO4 and RGF3-RHO4 are required for the formation of the contractile actomyosin ring and act downstream of BUD4}

To provide a potential hierarchy of action of BUD4, BUD3, RGF3, RHO4 and the formin BNI1 during septum formation in N. crassa, the dependencies for localization of these proteins in the different mutant backgrounds were determined. The formation of BNI1 rings was dependent on both GEFs, placing the two RHO4 functions upstream of the contractile actin ring. A similar observation was made in A. nidulans: the formin SepA was no longer recruited to septation sites in $\triangle A n b u d-3$ mutants. Furthermore, AnBud3 still accumulated at septation sites in the absence of SepA (Si et al., 2010). Rho GTPases are known to activate 
formins, which in turn control the assembly of the contractile actomyosin ring (e.g. Dong et al., 2003, Martin et al., 2007, Tolliday et al., 2001). Thus, RHO4 may control the assembly of the contractile actomyosin ring in $N$. crassa and in $A$. nidulans by regulating formin localization and activity.

Since in N. crassa both RHO4 modules function upstream of BNI1, it will be interesting to investigate whether BNI1 physically interacts with RHO4 thus being a downstream effector. For this purpose co-immunoprecipitation experiments with both proteins will be performed. Moreover, in case of an interaction between both proteins, it will be analyzed, which of both RHO4 modules activates BNI1.

BUD4 localized as cortical rings in $\Delta b u d-3$ and $\Delta r g f-3$. The accumulation of RGF3 as cortical ring was abolished in $\Delta r h o-4, \Delta b u d-3$ and $\Delta b u d-4$. Based on these data, it was concluded that RGF3 localization is dependent on all other proteins, and is thus likely acting after BUD3 and BUD4. The connection of BUD3 and BUD4 with RHO4 follows a more complex pattern: both BUD proteins are able to associate with the cortex as dots in $\Delta r h o-4$, and rare examples of BUD3 rings were also found. The formation of abundant BUD3 and BUD4 rings, however, was dependent on the presence of RHO4, indicating that ring formation, but not cortical localization of BUD3 and BUD4 required RHO4.

Consistent with the localization data, the following model is proposed (Fig. 21): the anillinrelated protein BUD4 acts as early landmark for the specification of future septation sites in $N$. crassa. This is in agreement with the site selection function described for its yeast counterparts Bud4p and Mid1p in budding and fission yeast, respectively (see section 4.4). Localization of BUD3 depends on BUD4, whose localizations lead to the recruitment and activation of RHO4. This may be reinforced by the GTPase itself and may provide a potential positive feedback loop for site selection and initiation of septation. The cooperation as a landmark providing the spatial cue for septation was also described for Bud3p and Bud4p in budding yeast and the Bud3 function in site selection is also conserved in A. gossypii and $A$. nidulans. Furthermore, the $A$. nidulans homologue AnBud3p also functions as Rho4-GEF (see sections 4.3 and 4.4).

A second function of RHO4 in N. crassa is controlled by RGF3, which is dependent on BUD4 and the BUD3-RHO4 module. Thus, this second RHO4 module is potentially acting later during the actual septation process. The formation of cortical BNI1 rings and subsequent actin rings may be driven by RGF3-activated RHO4. Similarly, the AnBud3-AnRho4 module in $A$. nidulans functions upstream of the formin SepA and is required for localization of SepA to the septation site (see section 4.7). Furthermore, the presence of BUD3, BUD4 and RHO4 
around the septal pore of mature septa suggests additional (marker) functions of these proteins (see section 4.5).

In the proposed model the septation initiation network (SIN) is suggested to be a potential upstream regulator of the BUD4-BUD3-RHO4-RGF3 signalling cascade, since in other organism such as fission yeast the SIN network is involved in controlling septation (see also the outlook section). This hypothesis is supported by the observation that the localization of AnBud3 to septation sites is dependent on a functional SIN pathway in A. nidulans (Si et al., 2010 ) and that the deletion mutants of the SIN components $d b f-2$ and $m o b-1$ in N. crassa lack septa indicating a function of these two genes in septation (Dvash et al., 2009, Maerz et al., 2009).

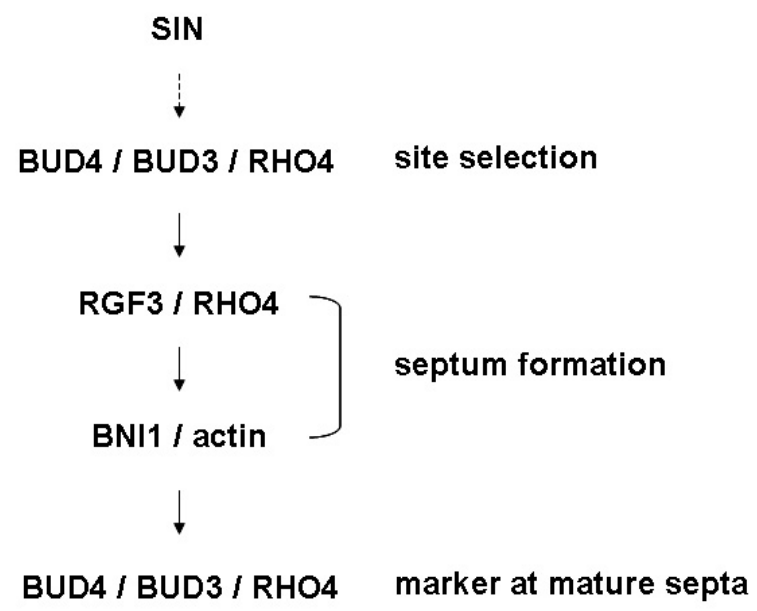

Fig. 21: Model summarizing the functions of the two RHO4 modules and their relationship to BUD4 during septum formation. The anillin-like protein BUD4 and the Rho GTPase RHO4 together with its GEF BUD3 may act as an early marker complex specifying future septation sites in $N$. crassa, whereas the second RHO4-GEF RGF3 may drive the BNI1-dependent septation process. The presence of BUD4, BUD3 and RHO4, but not RGF3, at the septal pore of mature septa suggests additional (marker) functions of these proteins. The SIN network is suggested as potential upstream regulator of this signalling cascade. For further details see text.

\subsection{Outlook}

In this study BUD3 and RGF3 were identified as RHO4-specific GEFs involved in septation. To investigate if both proteins also have redundant functions, the generation of a double knockout of bud-3 and rgf-3 was attempted, but no double mutant was obtained so far. Thus, a project for the near future is the generation of this double knockout mutant and its subsequent characterization.

The BUD4-BUD3-RHO4-RGF3 signalling cascade described above is far from being complete. Future work will need to focus on the extension of this network e.g. by identifying upstream regulators of BUD4 and thus of this network and downstream targets of both RHO4 
modules. For this purpose, co-purification experiments with the known network components followed by identification of their associated proteins via mass spectrometry are planned. Subsequently, co-immunoprecipitation studies will be performed to confirm the found interactions.

Interesting candidates, which may also be involved in this septation network, are the essential septin CDC12 and the PAK-like kinase CLA4. Both proteins localized to septa, but the GFP fusion proteins were not functional. Thus, the first step will be to confirm these preliminary localization data in wild type and subsequently in selected mutants. Moreover, additional genes were identified as essential for septum formation in N. crassa in the screen and thus are likely involved in the septation process. The two genes iqg-1 and cyk-3 belong to this group and it would be interesting to analyze their function in septation. In S. cerevisiae as well as in S. pombe the IQGAP protein is a component of the contractile actomyosin ring and is required for ring construction e.g. by binding to actin (Eng et al., 1998, Lippincott \& Li, 1998b, Shannon \& Li, 1999). Furthermore, in budding yeast Iqg1p interacts with Bud4p and the septin Cdc12p and is required for proper Bud4p localization (Osman et al., 2002). Thus, IQG1 in $N$. crassa could also interact with BUD4 thereby recruiting it to the septation site. However, IQG1 may also function downstream of the landmark complex BUD3-BUD4. This idea is supported by the observation in Ashbya that the localization of the IQGAP protein AgCyk1 is restricted to septation sites by AgBud3 (Wendland, 2003). Cyk3p in budding yeast is involved in the formation of the primary septum. It is thought to activate in cooperation with Inn1p the chitin synthase Chs2p for septum formation (Jendretzki et al., 2009, Korinek et al., 2000, Nishihama et al., 2009). Moreover, Cyk3p seems to act downstream of Iqg1p and is involved in the alternative cytokinesis pathway in budding yeast, which is independent of actomyosin ring formation (Jendretzki et al., 2009, Korinek et al., 2000). Maybe the N. crassa CYK3 functions in the formation of the septum by activation of chitin synthases. In Neurospora chitin deposition is coordinated with actomyosin ring constriction. Since both RHO4 modules are necessary for actin ring formation, they may also be involved in coordinating ring constriction with septum formation. Thus, CYK3 could be an effector protein of one of the two RHO4 modules.

There is evidence that the SIN/MEN network is conserved throughout the fungal kingdom and that it is involved in controlling septation. In S. pombe, for example, SIN coordinates cytokinesis with mitosis (Gould \& Simanis, 1997, Krapp \& Simanis, 2008, Simanis, 2003, Wolfe \& Gould, 2005) and in A. nidulans the SIN was shown to be essential for the formation of the contractile actomyosin ring (Bruno et al., 2001, Kim et al., 2006). In N. crassa the SIN 
is also likely involved in septation indicated by the phenotype of mutants deleted in $d b f-2$ and $m o b-1$, which lack septa. DBF2 and MOB1 are the sole SIN components described in $N$. crassa so far (Dvash et al., 2009, Maerz et al., 2009). In S. cerevisiae Dbf2p acts downstream of the kinase Cdc15p and is not only involved in mitotic exit, but also in cytokinesis (Frenz et al., 2000, Yoshida \& Toh-e, 2001). The same functions are described for the Dbf2p homologue in C. albicans (Gonzalez-Novo et al., 2009a). Moreover, the DBF2 counterpart in S. pombe $\operatorname{Sid} 2 \mathrm{p}$ promotes septum formation and upon its deletion cells are elongated and multinucleated (reviewed in Krapp \& Simanis, 2008). Thus, DBF2 is a good candidate for an upstream regulator of the BUD4-BUD3-RHO4-RGF3 signalling cascade. Consistently, in $A$. nidulans the localization of $\mathrm{AnBud} 3$ to septation sites is dependent on a functional SIN pathway (Si et al., 2010). In mutants lacking functional SepH, which is the A. nidulans homologue of the most upstream SIN kinase Cdc7p in S. pombe, AnBud3 was not recruited to septation sites, placing the SIN pathway upstream of AnBud3 function (Si et al., 2010). To investigate if this is also true in N. crassa, the localization pattern of both BUD proteins in the $d b f-2$ background and vice versa will be determined. Furthermore, to get more insights into the hierarchical organization of DBF2 and the BUD4-BUD3-RHO4-RGF3 signalling cascade a hyper-active mutant of DBF2 DBF2 ${ }^{\mathrm{T} 671 \mathrm{E}}$ based on observations made with the SIN kinase Sid2p in S. pombe (Hou et al., 2004) was generated. DBF2 ${ }^{\text {T671E }}$ was expressed in wild-type background and its hyper-activity (a three-fold stimulation compared to the wild type control) was validated in in vitro kinase assays performed by Dr. Sabine März (Institute of Microbiology and Genetics, University of Göttingen, D). Future work will need to address, if expression of hyper-active DBF2 can overcome the septation defect in the bud-3 and bud-4 deletion strains. This would indicate a DBF2 function downstream of the network. A further experiment will be to express dominant-active RHO4 in $\Delta d b f-2$. The suppression of the mutant defects would point at an upstream function of the kinase. 


\section{References}

Abe, K., K. L. Rossman, B. Liu, K. D. Ritola, D. Chiang, S. L. Campbell, K. Burridge \& C. J. Der, (2000) Vav2 is an activator of Cdc42, Rac1, and RhoA. J Biol Chem 275: 1014110149.

Almonacid, M., J. B. Moseley, J. Janvore, A. Mayeux, V. Fraisier, P. Nurse \& A. Paoletti, (2009) Spatial control of cytokinesis by Cdr2 kinase and Mid1/anillin nuclear export. Curr Biol 19: 961-966.

Araujo-Palomares, C. L., E. Castro-Longoria \& M. Riquelme, (2007) Ontogeny of the Spitzenkorper in germlings of Neurospora crassa. Fungal Genet Biol 44: 492-503.

Archambault, V. \& D. M. Glover, (2009) Polo-like kinases: conservation and divergence in their functions and regulation. Nat Rev Mol Cell Biol 10: 265-275.

Asakawa, K., S. Yoshida, F. Otake \& A. Toh-e, (2001) A novel functional domain of Cdc15 kinase is required for its interaction with Tem1 GTPase in Saccharomyces cerevisiae. Genetics 157: 1437-1450.

Ausübel, F., R. Brent, R. Kingston, D. Moore, J. Seidman, J. Smith, K. Struhl, L. Albright, D. Coen \& A. Varki, (1997) Current Protocols in Molecular Biology. John Wiley \& Sons, New York.

Ayad-Durieux, Y., P. Knechtle, S. Goff, F. Dietrich \& P. Philippsen, (2000) A PAK-like protein kinase is required for maturation of young hyphae and septation in the filamentous ascomycete Ashbya gossypii. J Cell Sci 113 Pt 24: 4563-4575.

Bahler, J., A. B. Steever, S. Wheatley, Y. Wang, J. R. Pringle, K. L. Gould \& D. McCollum, (1998) Role of polo kinase and Mid1p in determining the site of cell division in fission yeast. J Cell Biol 143: 1603-1616.

Bailly, E., S. Cabantous, D. Sondaz, A. Bernadac \& M. N. Simon, (2003) Differential cellular localization among mitotic cyclins from Saccharomyces cerevisiae: a new role for the axial budding protein Bud3 in targeting Clb2 to the mother-bud neck. J Cell Sci 116: 4119-4130.

Balasubramanian, M. K., E. Bi \& M. Glotzer, (2004) Comparative analysis of cytokinesis in budding yeast, fission yeast and animal cells. Curr Biol 14: R806-818.

Balasubramanian, M. K., D. M. Helfman \& S. M. Hemmingsen, (1992) A new tropomyosin essential for cytokinesis in the fission yeast S. pombe. Nature 360: 84-87.

Balasubramanian, M. K., B. R. Hirani, J. D. Burke \& K. L. Gould, (1994) The Schizosaccharomyces pombe cdc3+ gene encodes a profilin essential for cytokinesis. $J$ Cell Biol 125: 1289-1301.

Balasubramanian, M. K., D. McCollum, L. Chang, K. C. Wong, N. I. Naqvi, X. He, S. Sazer \& K. L. Gould, (1998) Isolation and characterization of new fission yeast cytokinesis mutants. Genetics 149: 1265-1275.

Banuett, F., R. H. Quintanilla, Jr. \& C. G. Reynaga-Pena, (2008) The machinery for cell polarity, cell morphogenesis, and the cytoskeleton in the Basidiomycete fungus Ustilago maydis-a survey of the genome sequence. Fungal Genet Biol 45 Suppl 1: S3S14.

Barale, S., D. McCusker \& R. A. Arkowitz, (2006) Cdc42p GDP/GTP cycling is necessary for efficient cell fusion during yeast mating. Mol Biol Cell 17: 2824-2838.

Bardin, A. J. \& A. Amon, (2001) Men and sin: what's the difference? Nat Rev Mol Cell Biol 2: $815-826$.

Bardin, A. J., R. Visintin \& A. Amon, (2000) A mechanism for coupling exit from mitosis to partitioning of the nucleus. Cell 102: 21-31.

Barr, F. A. \& U. Gruneberg, (2007) Cytokinesis: placing and making the final cut. Cell 131: 847-860. 
Becker, D. M. \& V. Lundblad, (2001) Introduction of DNA into yeast cells. Curr Protoc Mol Biol Chapter 13: Unit13 17.

Bement, W. M., H. A. Benink \& G. von Dassow, (2005) A microtubule-dependent zone of active RhoA during cleavage plane specification. J Cell Biol 170: 91-101.

Bement, W. M., A. L. Miller \& G. von Dassow, (2006) Rho GTPase activity zones and transient contractile arrays. Bioessays 28: 983-993.

Bender, A. \& J. R. Pringle, (1989) Multicopy suppression of the cdc24 budding defect in yeast by $\mathrm{CDC} 42$ and three newly identified genes including the ras-related gene RSR1. Proc Natl Acad Sci U S A 86: 9976-9980.

Benink, H. A. \& W. M. Bement, (2005) Concentric zones of active RhoA and Cdc42 around single cell wounds. J Cell Biol 168: 429-439.

Berlin, A., A. Paoletti \& F. Chang, (2003) Mid2p stabilizes septin rings during cytokinesis in fission yeast. J Cell Biol 160: 1083-1092.

Bertani, G., (1951) Studies on lysogenesis. I. The mode of phage liberation by lysogenic Escherichia coli. J Bacteriol 62: 293-300.

Bezanilla, M., S. L. Forsburg \& T. D. Pollard, (1997) Identification of a second myosin-II in Schizosaccharomyces pombe: Myp2p is conditionally required for cytokinesis. Mol Biol Cell 8: 2693-2705.

Bi, E., P. Maddox, D. J. Lew, E. D. Salmon, J. N. McMillan, E. Yeh \& J. R. Pringle, (1998) Involvement of an actomyosin contractile ring in Saccharomyces cerevisiae cytokinesis. J Cell Biol 142: 1301-1312.

Borkovich, K. A., L. A. Alex, O. Yarden, M. Freitag, G. E. Turner, N. D. Read, S. Seiler, D. Bell-Pedersen, J. Paietta, N. Plesofsky, M. Plamann, M. Goodrich-Tanrikulu, U. Schulte, G. Mannhaupt, F. E. Nargang, A. Radford, C. Selitrennikoff, J. E. Galagan, J. C. Dunlap, J. J. Loros, D. Catcheside, H. Inoue, R. Aramayo, M. Polymenis, E. U. Selker, M. S. Sachs, G. A. Marzluf, I. Paulsen, R. Davis, D. J. Ebbole, A. Zelter, E. R. Kalkman, R. O'Rourke, F. Bowring, J. Yeadon, C. Ishii, K. Suzuki, W. Sakai \& R. Pratt, (2004) Lessons from the genome sequence of Neurospora crassa: tracing the path from genomic blueprint to multicellular organism. Microbiol Mol Biol Rev 68: 1108.

Bradford, M. M., (1976) A rapid and sensitive method for the quantitation of microgram quantities of protein utilizing the principle of protein-dye binding. Anal Biochem 72: 248-254.

Brandt, D. T. \& R. Grosse, (2007) Get to grips: steering local actin dynamics with IQGAPs. EMBO Rep 8: 1019-1023.

Bruno, K. S., J. L. Morrell, J. E. Hamer \& C. J. Staiger, (2001) SEPH, a Cdc7p orthologue from Aspergillus nidulans, functions upstream of actin ring formation during cytokinesis. Mol Microbiol 42: 3-12.

Buchsbaum, R. J., (2007) Rho activation at a glance. J Cell Sci 120: 1149-1152.

Carnahan, R. H. \& K. L. Gould, (2003) The PCH family protein, Cdc15p, recruits two F-actin nucleation pathways to coordinate cytokinetic actin ring formation in Schizosaccharomyces pombe. J Cell Biol 162: 851-862.

Casamayor, A. \& M. Snyder, (2002) Bud-site selection and cell polarity in budding yeast. Curr Opin Microbiol 5: 179-186.

Celton-Morizur, S., V. Racine, J. B. Sibarita \& A. Paoletti, (2006) Pom1 kinase links division plane position to cell polarity by regulating Mid1p cortical distribution. J Cell Sci 119: 4710-4718.

Chang, F., D. Drubin \& P. Nurse, (1997) cdc12p, a protein required for cytokinesis in fission yeast, is a component of the cell division ring and interacts with profilin. J Cell Biol 137: 169-182. 
Chang, F., A. Woollard \& P. Nurse, (1996) Isolation and characterization of fission yeast mutants defective in the assembly and placement of the contractile actin ring. $J$ Cell Sci 109 ( Pt 1): 131-142.

Chant, J., K. Corrado, J. R. Pringle \& I. Herskowitz, (1991) Yeast BUD5, encoding a putative GDP-GTP exchange factor, is necessary for bud site selection and interacts with bud formation gene BEM1. Cell 65: 1213-1224.

Chant, J. \& I. Herskowitz, (1991) Genetic control of bud site selection in yeast by a set of gene products that constitute a morphogenetic pathway. Cell 65: 1203-1212.

Chant, J., M. Mischke, E. Mitchell, I. Herskowitz \& J. R. Pringle, (1995) Role of Bud3p in producing the axial budding pattern of yeast. $J$ Cell Biol 129: 767-778.

Chen, C. T., A. Feoktistova, J. S. Chen, Y. S. Shim, D. M. Clifford, K. L. Gould \& D. McCollum, (2008) The SIN kinase Sid2 regulates cytoplasmic retention of the $S$. pombe Cdc14-like phosphatase Clp1. Curr Biol 18: 1594-1599.

Chesarone, M. A., A. G. DuPage \& B. L. Goode, (2010) Unleashing formins to remodel the actin and microtubule cytoskeletons. Nat Rev Mol Cell Biol 11: 62-74.

Christianson, T. W., R. S. Sikorski, M. Dante, J. H. Shero \& P. Hieter, (1992) Multifunctional yeast high-copy-number shuttle vectors. Gene 110: 119-122.

Chuang, J. S. \& R. W. Schekman, (1996) Differential trafficking and timed localization of two chitin synthase proteins, Chs2p and Chs3p. J Cell Biol 135: 597-610.

Clifford, D. M., C. T. Chen, R. H. Roberts, A. Feoktistova, B. A. Wolfe, J. S. Chen, D. McCollum \& K. L. Gould, (2008) The role of Cdc14 phosphatases in the control of cell division. Biochem Soc Trans 36: 436-438.

Colot, H. V., G. Park, G. E. Turner, C. Ringelberg, C. M. Crew, L. Litvinkova, R. L. Weiss, K. A. Borkovich \& J. C. Dunlap, (2006) A high-throughput gene knockout procedure for Neurospora reveals functions for multiple transcription factors. Proc Natl Acad Sci US A 103: 10352-10357.

Corpet, F., (1988) Multiple sequence alignment with hierarchical clustering. Nucleic Acids Res 16: 10881-10890.

Cote, J. F. \& K. Vuori, (2007) GEF what? Dock180 and related proteins help Rac to polarize cells in new ways. Trends Cell Biol 17: 383-393.

Cvrckova, F., C. De Virgilio, E. Manser, J. R. Pringle \& K. Nasmyth, (1995) Ste20-like protein kinases are required for normal localization of cell growth and for cytokinesis in budding yeast. Genes Dev 9: 1817-1830.

D'Avino, P. P., (2009) How to scaffold the contractile ring for a safe cytokinesis - lessons from Anillin-related proteins. J Cell Sci 122: 1071-1079.

D'Avino, P. P. \& D. M. Glover, (2009) Cytokinesis: mind the GAP. Nat Cell Biol 11: 112114.

D'Avino, P. P., T. Takeda, L. Capalbo, W. Zhang, K. S. Lilley, E. D. Laue \& D. M. Glover, (2008) Interaction between Anillin and RacGAP50C connects the actomyosin contractile ring with spindle microtubules at the cell division site. J Cell Sci 121: 1151-1158.

Daga, R. R. \& F. Chang, (2005) Dynamic positioning of the fission yeast cell division plane. Proc Natl Acad Sci U S A 102: 8228-8232.

DeMarini, D. J., A. E. Adams, H. Fares, C. De Virgilio, G. Valle, J. S. Chuang \& J. R. Pringle, (1997) A septin-based hierarchy of proteins required for localized deposition of chitin in the Saccharomyces cerevisiae cell wall. J Cell Biol 139: 75-93.

DerMardirossian, C. \& G. M. Bokoch, (2005) GDIs: central regulatory molecules in Rho GTPase activation. Trends Cell Biol 15: 356-363.

Dietrich, F. S., S. Voegeli, S. Brachat, A. Lerch, K. Gates, S. Steiner, C. Mohr, R. Pohlmann, P. Luedi, S. Choi, R. A. Wing, A. Flavier, T. D. Gaffney \& P. Philippsen, (2004) The 
Ashbya gossypii genome as a tool for mapping the ancient Saccharomyces cerevisiae genome. Science 304: 304-307.

Dobbelaere, J. \& Y. Barral, (2004) Spatial coordination of cytokinetic events by compartmentalization of the cell cortex. Science 305: 393-396.

Dong, Y., D. Pruyne \& A. Bretscher, (2003) Formin-dependent actin assembly is regulated by distinct modes of Rho signaling in yeast. J Cell Biol 161: 1081-1092.

Doonan, J. H. \& G. Kitsios, (2009) Functional evolution of cyclin-dependent kinases. Mol Biotechnol 42: 14-29.

Dovas, A. \& J. R. Couchman, (2005) RhoGDI: multiple functions in the regulation of Rho family GTPase activities. Biochem J390: 1-9.

Drechsel, D. N., A. A. Hyman, A. Hall \& M. Glotzer, (1997) A requirement for Rho and Cdc42 during cytokinesis in Xenopus embryos. Curr Biol 7: 12-23.

Dunkler, A. \& J. Wendland, (2007) Candida albicans Rho-type GTPase-encoding genes required for polarized cell growth and cell separation. Eukaryot Cell 6: 844-854.

Dunlap, J. C., K. A. Borkovich, M. R. Henn, G. E. Turner, M. S. Sachs, N. L. Glass, K. McCluskey, M. Plamann, J. E. Galagan, B. W. Birren, R. L. Weiss, J. P. Townsend, J. J. Loros, M. A. Nelson, R. Lambreghts, H. V. Colot, G. Park, P. Collopy, C. Ringelberg, C. Crew, L. Litvinkova, D. DeCaprio, H. M. Hood, S. Curilla, M. Shi, M. Crawford, M. Koerhsen, P. Montgomery, L. Larson, M. Pearson, T. Kasuga, C. Tian, M. Basturkmen, L. Altamirano \& J. Xu, (2007) Enabling a community to dissect an organism: overview of the Neurospora functional genomics project. Adv Genet 57: 4996.

Dutartre, H., J. Davoust, J. P. Gorvel \& P. Chavrier, (1996) Cytokinesis arrest and redistribution of actin-cytoskeleton regulatory components in cells expressing the Rho GTPase CDC42Hs. J Cell Sci 109 ( Pt 2): 367-377.

Dvash, E., G. Kra-Oz, C. Ziv, S. Carmeli \& O. Yarden, (2009) The NDR kinase DBF-2 is Involved in Regulation of Mitosis, Conidial Development and Glycogen Metabolism in Neurospora crassa. Eukaryot Cell.

Dynesen, J. \& J. Nielsen, (2003) Branching is coordinated with mitosis in growing hyphae of Aspergillus nidulans. Fungal Genet Biol 40: 15-24.

Eggert, U. S., T. J. Mitchison \& C. M. Field, (2006) Animal cytokinesis: from parts list to mechanisms. Annu Rev Biochem 75: 543-566.

Eme, L., D. Moreira, E. Talla \& C. Brochier-Armanet, (2009) A complex cell division machinery was present in the last common ancestor of eukaryotes. PLoS One 4: e5021.

Eng, K., N. I. Naqvi, K. C. Wong \& M. K. Balasubramanian, (1998) Rng2p, a protein required for cytokinesis in fission yeast, is a component of the actomyosin ring and the spindle pole body. Curr Biol 8: 611-621.

Etienne-Manneville, S. \& A. Hall, (2002) Rho GTPases in cell biology. Nature 420: 629-635.

Fankhauser, C., A. Reymond, L. Cerutti, S. Utzig, K. Hofmann \& V. Simanis, (1995) The $S$. pombe cdc15 gene is a key element in the reorganization of F-actin at mitosis. Cell $\mathbf{8 2}$ : 435-444.

Fankhauser, C. \& V. Simanis, (1994) The cdc7 protein kinase is a dosage dependent regulator of septum formation in fission yeast. EMBOJ 13: 3011-3019.

Fidyk, N., J. B. Wang \& R. A. Cerione, (2006) Influencing cellular transformation by modulating the rates of GTP hydrolysis by Cdc42. Biochemistry 45: 7750-7762.

Field, C., R. Li \& K. Oegema, (1999) Cytokinesis in eukaryotes: a mechanistic comparison. Curr Opin Cell Biol 11: 68-80.

Fleissner, A., A. C. Leeder, M. G. Roca, N. D. Read \& N. L. Glass, (2009) Oscillatory recruitment of signaling proteins to cell tips promotes coordinated behavior during cell fusion. Proc Natl Acad Sci US A 106: 19387-19392. 
Freitag, M., P. C. Hickey, N. B. Raju, E. U. Selker \& N. D. Read, (2004) GFP as a tool to analyze the organization, dynamics and function of nuclei and microtubules in Neurospora crassa. Fungal Genet Biol 41: 897-910.

Frenz, L. M., S. E. Lee, D. Fesquet \& L. H. Johnston, (2000) The budding yeast Dbf2 protein kinase localises to the centrosome and moves to the bud neck in late mitosis. J Cell Sci 113 Pt 19: 3399-3408.

Fujita, A., C. Oka, Y. Arikawa, T. Katagai, A. Tonouchi, S. Kuhara \& Y. Misumi, (1994) A yeast gene necessary for bud-site selection encodes a protein similar to insulindegrading enzymes. Nature 372: 567-570.

Gale, C., M. Gerami-Nejad, M. McClellan, S. Vandoninck, M. S. Longtine \& J. Berman, (2001) Candida albicans Int1p interacts with the septin ring in yeast and hyphal cells. Mol Biol Cell 12: 3538-3549.

Gao, X. D., L. M. Sperber, S. A. Kane, Z. Tong, A. H. Tong, C. Boone \& E. Bi, (2007) Sequential and distinct roles of the cadherin domain-containing protein Axl2p in cell polarization in yeast cell cycle. Mol Biol Cell 18: 2542-2560.

Garcia-Pedrajas, M. D., M. Nadal, M. Bolker, S. E. Gold \& M. H. Perlin, (2008) Sending mixed signals: redundancy vs. uniqueness of signaling components in the plant pathogen, Ustilago maydis. Fungal Genet Biol 45 Suppl 1: S22-30.

Garcia, P., V. Tajadura, I. Garcia \& Y. Sanchez, (2006) Rgflp is a specific Rho1-GEF that coordinates cell polarization with cell wall biogenesis in fission yeast. Mol Biol Cell 17: $1620-1631$.

Gladfelter, A. \& J. Berman, (2009) Dancing genomes: fungal nuclear positioning. Nat Rev Microbiol 7: 875-886.

Gladfelter, A. S., (2006) Nuclear anarchy: asynchronous mitosis in multinucleated fungal hyphae. Curr Opin Microbiol 9: 547-552.

Gladfelter, A. S., L. Kozubowski, T. R. Zyla \& D. J. Lew, (2005) Interplay between septin organization, cell cycle and cell shape in yeast. J Cell Sci 118: 1617-1628.

Gladfelter, A. S., J. R. Pringle \& D. J. Lew, (2001) The septin cortex at the yeast mother-bud neck. Curr Opin Microbiol 4: 681-689.

Glotzer, M., (2001) Animal cell cytokinesis. Annu Rev Cell Dev Biol 17: 351-386.

Gonzalez-Novo, A., J. Jimenez, M. J. Garcia, I. Rios-Serrano, J. Pla, A. Jimenez \& M. Sanchez-Perez, (2004) Dynamics of CaCdc10, a septin of Candida albicans, in living cells and during infection. Int Microbiol 7: 105-112.

Gonzalez-Novo, A., L. Labrador, M. E. Pablo-Hernando, J. Correa-Bordes, M. Sanchez, J. Jimenez \& C. R. Vazquez de Aldana, (2009a) Dbf2 is essential for cytokinesis and correct mitotic spindle formation in Candida albicans. Mol Microbiol 72: 1364-1378.

Gonzalez-Novo, A., C. R. Vazquez de Aldana \& A. Jimenez, (2009b) Fungal septins: one ring to rule it all? Central European Journal of Biology 4: 274-289.

Gould, K. L. \& V. Simanis, (1997) The control of septum formation in fission yeast. Genes Dev 11: 2939-2951.

Gregory, S. L., S. Ebrahimi, J. Milverton, W. M. Jones, A. Bejsovec \& R. Saint, (2008) Cell division requires a direct link between microtubule-bound RacGAP and Anillin in the contractile ring. Curr Biol 18: 25-29.

Guertin, D. A., S. Trautmann \& D. McCollum, (2002) Cytokinesis in eukaryotes. Microbiol Mol Biol Rev 66: 155-178.

Hachet, O. \& V. Simanis, (2008) Mid1p/anillin and the septation initiation network orchestrate contractile ring assembly for cytokinesis. Genes Dev 22: 3205-3216.

Hall, A., (2005) Rho GTPases and the control of cell behaviour. Biochem Soc Trans 33: 891895. 
Harkins, H. A., N. Page, L. R. Schenkman, C. De Virgilio, S. Shaw, H. Bussey \& J. R. Pringle, (2001) Bud8p and Bud9p, proteins that may mark the sites for bipolar budding in yeast. Mol Biol Cell 12: 2497-2518.

Harris, S. D., (2001) Septum formation in Aspergillus nidulans. Curr Opin Microbiol 4: 736739.

Harris, S. D., L. Hamer, K. E. Sharpless \& J. E. Hamer, (1997) The Aspergillus nidulans sepA gene encodes an FH1/2 protein involved in cytokinesis and the maintenance of cellular polarity. EMBO J 16: 3474-3483.

Harris, S. D., J. L. Morrell \& J. E. Hamer, (1994) Identification and characterization of Aspergillus nidulans mutants defective in cytokinesis. Genetics 136: 517-532.

Harris, S. D., N. D. Read, R. W. Roberson, B. Shaw, S. Seiler, M. Plamann \& M. Momany, (2005) Polarisome meets spitzenkorper: microscopy, genetics, and genomics converge. Eukaryot Cell 4: 225-229.

Harris, S. D., G. Turner, V. Meyer, E. A. Espeso, T. Specht, N. Takeshita \& K. Helmstedt, (2009) Morphology and development in Aspergillus nidulans: a complex puzzle. Fungal Genet Biol 46 Suppl 1: S82-S92.

Helfer, H. \& A. S. Gladfelter, (2006) AgSwelp regulates mitosis in response to morphogenesis and nutrients in multinucleated Ashbya gossypii cells. Mol Biol Cell 17: 4494-4512.

Hickey, P. C., D. J. Jacobson, N. D. Read \& N. Louise Glass, (2002) Live-cell imaging of vegetative hyphal fusion in Neurospora crassa. Fungal Genetics and Biology 37: 109119.

Honda, S. \& E. U. Selker, (2009) Tools for fungal proteomics: multifunctional neurospora vectors for gene replacement, protein expression and protein purification. Genetics 182: 11-23.

Hou, M. C., D. A. Guertin \& D. McCollum, (2004) Initiation of cytokinesis is controlled through multiple modes of regulation of the Sid2p-Mob1p kinase complex. Mol Cell Biol 24: 3262-3276.

Hu, F., Y. Wang, D. Liu, Y. Li, J. Qin \& S. J. Elledge, (2001) Regulation of the Bub2/Bfa1 GAP complex by Cdc5 and cell cycle checkpoints. Cell 107: 655-665.

Huang, Y., T. G. Chew, W. Ge \& M. K. Balasubramanian, (2007) Polarity determinants Tealp, Tea $4 p$, and Pom $1 p$ inhibit division-septum assembly at cell ends in fission yeast. Dev Cell 12: 987-996.

Hwa Lim, H., F. M. Yeong \& U. Surana, (2003) Inactivation of mitotic kinase triggers translocation of MEN components to mother-daughter neck in yeast. Mol Biol Cell 14: 4734-4743.

Iden, S. \& J. G. Collard, (2008) Crosstalk between small GTPases and polarity proteins in cell polarization. Nat Rev Mol Cell Biol 9: 846-859.

Imai, J., A. Toh-e \& Y. Matsui, (1996) Genetic analysis of the Saccharomyces cerevisiae RHO3 gene, encoding a rho-type small GTPase, provides evidence for a role in bud formation. Genetics 142: 359-369.

Inoue, H., H. Nojima \& H. Okayama, (1990) High efficiency transformation of Escherichia coli with plasmids. Gene 96: 23-28.

Irazoqui, J. E., A. S. Gladfelter \& D. J. Lew, (2003) Scaffold-mediated symmetry breaking by Cdc42p. Nat Cell Biol 5: 1062-1070.

Iwase, M., J. Luo, S. Nagaraj, M. Longtine, H. B. Kim, B. K. Haarer, C. Caruso, Z. Tong, J. R. Pringle \& E. Bi, (2006) Role of a Cdc42p effector pathway in recruitment of the yeast septins to the presumptive bud site. Mol Biol Cell 17: 1110-1125.

James, P., J. Halladay \& E. A. Craig, (1996) Genomic libraries and a host strain designed for highly efficient two-hybrid selection in yeast. Genetics 144: 1425-1436. 
Jedd, G. \& N. H. Chua, (2000) A new self-assembled peroxisomal vesicle required for efficient resealing of the plasma membrane. Nat Cell Biol 2: 226-231.

Jendretzki, A., I. Ciklic, R. Rodicio, H. P. Schmitz \& J. J. Heinisch, (2009) Cyk3 acts in actomyosin ring independent cytokinesis by recruiting Inn1 to the yeast bud neck. Mol Genet Genomics 282: 437-451.

Jimenez, J., V. J. Cid, R. Cenamor, M. Yuste, G. Molero, C. Nombela \& M. Sanchez, (1998) Morphogenesis beyond cytokinetic arrest in Saccharomyces cerevisiae. J Cell Biol 143: $1617-1634$.

Jin, Q. W., M. Zhou, A. Bimbo, M. K. Balasubramanian \& D. McCollum, (2006) A role for the septation initiation network in septum assembly revealed by genetic analysis of sid2-250 suppressors. Genetics 172: 2101-2112.

Jones, T., N. A. Federspiel, H. Chibana, J. Dungan, S. Kalman, B. B. Magee, G. Newport, Y. R. Thorstenson, N. Agabian, P. T. Magee, R. W. Davis \& S. Scherer, (2004) The diploid genome sequence of Candida albicans. Proc Natl Acad Sci U S A 101: 73297334.

Justa-Schuch, D., Y. Heilig, C. Richthammer \& S. Seiler, Septum formation is regulated by the RHO4-specific exchange factors BUD3 and RGF3 and by the landmark protein BUD4 in Neurospora crassa. Mol Microbiol. 2010 Feb. 28. [Epub ahead of print]. doi:10.1111/j.1365-2958.2010.07093x.

Kamei, T., K. Tanaka, T. Hihara, M. Umikawa, H. Imamura, M. Kikyo, K. Ozaki \& Y. Takai, (1998) Interaction of Bnrlp with a novel Src homology 3 domain-containing Hoflp. Implication in cytokinesis in Saccharomyces cerevisiae. J Biol Chem 273: 2834128345.

Kaminskyj, S. G., (2000) Septum position is marked at the tip of Aspergillus nidulans hyphae. Fungal Genet Biol 31: 105-113.

Kaminskyj, S. G. \& J. E. Hamer, (1998) hyp loci control cell pattern formation in the vegetative mycelium of Aspergillus nidulans. Genetics 148: 669-680.

Kang, P. J., E. Angerman, K. Nakashima, J. R. Pringle \& H. O. Park, (2004) Interactions among Rax 1p, Rax2p, Bud8p, and Bud9p in marking cortical sites for bipolar bud-site selection in yeast. Mol Biol Cell 15: 5145-5157.

Kang, P. J., A. Sanson, B. Lee \& H. O. Park, (2001) A GDP/GTP exchange factor involved in linking a spatial landmark to cell polarity. Science 292: 1376-1378.

Kaufmann, A. \& P. Philippsen, (2009) Of bars and rings: Hof1-dependent cytokinesis in multiseptated hyphae of Ashbya gossypii. Mol Cell Biol 29: 771-783.

Kim, J. M., L. Lu, R. Shao, J. Chin \& B. Liu, (2006) Isolation of mutations that bypass the requirement of the septation initiation network for septum formation and conidiation in Aspergillus nidulans. Genetics 173: 685-696.

Kim, J. M., C. J. Zeng, T. Nayak, R. Shao, A. C. Huang, B. R. Oakley \& B. Liu, (2009) Timely septation requires SNAD-dependent spindle pole body localization of the septation initiation network components in the filamentous fungus Aspergillus nidulans. Mol Biol Cell 20: 2874-2884.

Knechtle, P., F. Dietrich \& P. Philippsen, (2003) Maximal polar growth potential depends on the polarisome component AgSpa2 in the filamentous fungus Ashbya gossypii. Mol Biol Cell 14: 4140-4154.

Kono, K., S. Nogami, M. Abe, M. Nishizawa, S. Morishita, D. Pellman \& Y. Ohya, (2008) G1/S cyclin-dependent kinase regulates small GTPase Rholp through phosphorylation of RhoGEF Tus1p in Saccharomyces cerevisiae. Mol Biol Cell 19: 1763-1771.

Korinek, W. S., E. Bi, J. A. Epp, L. Wang, J. Ho \& J. Chant, (2000) Cyk3, a novel SH3domain protein, affects cytokinesis in yeast. Curr Biol 10: 947-950. 
Kovar, D. R., J. R. Kuhn, A. L. Tichy \& T. D. Pollard, (2003) The fission yeast cytokinesis formin $\mathrm{Cdc} 12 \mathrm{p}$ is a barbed end actin filament capping protein gated by profilin. $J$ Cell Biol 161: 875-887.

Kovar, D. R., J. Q. Wu \& T. D. Pollard, (2005) Profilin-mediated competition between capping protein and formin Cdc12p during cytokinesis in fission yeast. Mol Biol Cell 16: $2313-2324$.

Kozminski, K. G., L. Beven, E. Angerman, A. H. Tong, C. Boone \& H. O. Park, (2003) Interaction between a Ras and a Rho GTPase couples selection of a growth site to the development of cell polarity in yeast. Mol Biol Cell 14: 4958-4970.

Krapp, A. \& V. Simanis, (2008) An overview of the fission yeast septation initiation network (SIN). Biochem Soc Trans 36: 411-415.

Le Goff, X., F. Motegi, E. Salimova, I. Mabuchi \& V. Simanis, (2000) The S. pombe rlc1 gene encodes a putative myosin regulatory light chain that binds the type II myosins myo3p and myo2p. J Cell Sci 113 Pt 23: 4157-4163.

Lee, J., H. S. Hwang, J. Kim \& K. Song, (1999) Ibd1p, a possible spindle pole body associated protein, regulates nuclear division and bud separation in Saccharomyces cerevisiae. Biochim Biophys Acta 1449: 239-253.

Lee, S. E., L. M. Frenz, N. J. Wells, A. L. Johnson \& L. H. Johnston, (2001a) Order of function of the budding-yeast mitotic exit-network proteins Tem1, Cdc15, Mob1, Dbf2, and Cdc5. Curr Biol 11: 784-788.

Lee, S. E., S. Jensen, L. M. Frenz, A. L. Johnson, D. Fesquet \& L. H. Johnston, (2001b) The Bub2-dependent mitotic pathway in yeast acts every cell cycle and regulates cytokinesis. J Cell Sci 114: 2345-2354.

Levin, D. E., (2005) Cell wall integrity signaling in Saccharomyces cerevisiae. Microbiol Mol Biol Rev 69: 262-291.

Li, C. R., Y. M. Wang \& Y. Wang, (2008) The IQGAP Iqg1 is a regulatory target of CDK for cytokinesis in Candida albicans. EMBO J 27: 2998-3010.

Lindsey, R., S. Cowden, Y. Hernandez-Rodriguez \& M. Momany, (2010) Septins AspA and AspC are important for normal development and limit the emergence of new growth foci in the multicellular fungus Aspergillus nidulans. Eukaryot Cell 9: 155-163.

Lippincott, J. \& R. Li, (1998a) Dual function of Cyk2, a cdc15/PSTPIP family protein, in regulating actomyosin ring dynamics and septin distribution. J Cell Biol 143: 19471960.

Lippincott, J. \& R. Li, (1998b) Sequential assembly of myosin II, an IQGAP-like protein, and filamentous actin to a ring structure involved in budding yeast cytokinesis. J Cell Biol 140: $355-366$.

Lippincott, J. \& R. Li, (2000) Involvement of PCH family proteins in cytokinesis and actin distribution. Microsc Res Tech 49: 168-172.

Lippincott, J., K. B. Shannon, W. Shou, R. J. Deshaies \& R. Li, (2001) The Tem1 small GTPase controls actomyosin and septin dynamics during cytokinesis. J Cell Sci 114: $1379-1386$.

Liu, J., X. Tang, H. Wang, S. Oliferenko \& M. K. Balasubramanian, (2002) The localization of the integral membrane protein Cps1p to the cell division site is dependent on the actomyosin ring and the septation-inducing network in Schizosaccharomyces pombe. Mol Biol Cell 13: 989-1000.

Longtine, M. S., D. J. DeMarini, M. L. Valencik, O. S. Al-Awar, H. Fares, C. De Virgilio \& J. R. Pringle, (1996) The septins: roles in cytokinesis and other processes. Curr Opin Cell Biol 8: 106-119.

Lord, M., F. Inose, T. Hiroko, T. Hata, A. Fujita \& J. Chant, (2002) Subcellular localization of Axl1, the cell type-specific regulator of polarity. Curr Biol 12: 1347-1352. 
Luca, F. C., M. Mody, C. Kurischko, D. M. Roof, T. H. Giddings \& M. Winey, (2001) Saccharomyces cerevisiae Mob1p is required for cytokinesis and mitotic exit. Mol Cell Biol 21: 6972-6983.

Madhani, H. D. \& G. R. Fink, (1998) The control of filamentous differentiation and virulence in fungi. Trends Cell Biol 8: 348-353.

Maerz, S., A. Dettmann, C. Ziv, Y. Liu, O. Valerius, O. Yarden \& S. Seiler, (2009) Two NDR kinase-MOB complexes function as distinct modules during septum formation and tip extension in Neurospora crassa. Mol Microbiol 74: 707-723.

Mah, A. S., J. Jang \& R. J. Deshaies, (2001) Protein kinase Cdc15 activates the Dbf2-Mob1 kinase complex. Proc Natl Acad Sci U S A 98: 7325-7330.

Martin, S. G., S. A. Rincon, R. Basu, P. Perez \& F. Chang, (2007) Regulation of the formin for3p by cdc42p and bud6p. Mol Biol Cell 18: 4155-4167.

Matsui, Y. \& E. A. Toh, (1992) Yeast RHO3 and RHO4 ras superfamily genes are necessary for bud growth, and their defect is suppressed by a high dose of bud formation genes CDC42 and BEM1. Mol Cell Biol 12: 5690-5699.

McCollum, D., M. K. Balasubramanian, L. E. Pelcher, S. M. Hemmingsen \& K. L. Gould, (1995) Schizosaccharomyces pombe cdc4+ gene encodes a novel EF-hand protein essential for cytokinesis. J Cell Biol 130: 651-660.

Merril, C. R., (1990) Gel-staining techniques. Methods Enzymol 182: 477-488.

Miller, P. J. \& D. I. Johnson, (1994) Cdc42p GTPase is involved in controlling polarized cell growth in Schizosaccharomyces pombe. Mol Cell Biol 14: 1075-1083.

Miller, R. K., D. Matheos \& M. D. Rose, (1999) The cortical localization of the microtubule orientation protein, Kar9p, is dependent upon actin and proteins required for polarization. J Cell Biol 144: 963-975.

Minet, M., P. Nurse, P. Thuriaux \& J. M. Mitchison, (1979) Uncontrolled septation in a cell division cycle mutant of the fission yeast Schizosaccharomyces pombe. J Bacteriol 137: 440-446.

Mishra, M., J. Karagiannis, M. Sevugan, P. Singh \& M. K. Balasubramanian, (2005) The 143-3 protein $\operatorname{rad} 24 p$ modulates function of the cdc14p family phosphatase clp $1 p / f l p 1 p$ in fission yeast. Curr Biol 15: 1376-1383.

Mishra, M., J. Karagiannis, S. Trautmann, H. Wang, D. McCollum \& M. K. Balasubramanian, (2004) The Clplp/Flp1p phosphatase ensures completion of cytokinesis in response to minor perturbation of the cell division machinery in Schizosaccharomyces pombe. J Cell Sci 117: 3897-3910.

Mishra, N. C. \& E. L. Tatum, (1972) Effect of L-sorbose on polysaccharide synthetases of Neurospora crassa (glycogen- -1,3-glucan-morphology-cell wall-digitonin-particulate enzymes). Proc Natl Acad Sci U S A 69: 313-317.

Mohl, D. A., M. J. Huddleston, T. S. Collingwood, R. S. Annan \& R. J. Deshaies, (2009) Dbf2-Mob1 drives relocalization of protein phosphatase Cdc14 to the cytoplasm during exit from mitosis. $J$ Cell Biol 184: 527-539.

Momany, M. \& J. E. Hamer, (1997) Relationship of actin, microtubules, and crosswall synthesis during septation in Aspergillus nidulans. Cell Motil Cytoskeleton 38: 373 384.

Momany, M., J. Zhao, R. Lindsey \& P. J. Westfall, (2001) Characterization of the Aspergillus nidulans septin (asp) gene family. Genetics 157: 969-977.

Morrell-Falvey, J. L., L. Ren, A. Feoktistova, G. D. Haese \& K. L. Gould, (2005) Cell wall remodeling at the fission yeast cell division site requires the Rho-GEF Rgf3p. J Cell Sci 118: 5563-5573.

Moseley, J. B., A. Mayeux, A. Paoletti \& P. Nurse, (2009) A spatial gradient coordinates cell size and mitotic entry in fission yeast. Nature 459: 857-860. 
Motegi, F., M. Mishra, M. K. Balasubramanian \& I. Mabuchi, (2004) Myosin-II reorganization during mitosis is controlled temporally by its dephosphorylation and spatially by Mid1 in fission yeast. $J$ Cell Biol 165: 685-695.

Mutoh, T., K. Nakano \& I. Mabuchi, (2005) Rho1-GEFs Rgf1 and Rgf2 are involved in formation of cell wall and septum, while Rgf3 is involved in cytokinesis in fission yeast. Genes Cells 10: 1189-1202.

Nakano, K., R. Arai \& I. Mabuchi, (1997) The small GTP-binding protein Rhol is a multifunctional protein that regulates actin localization, cell polarity, and septum formation in the fission yeast Schizosaccharomyces pombe. Genes Cells 2: 679-694.

Nakano, K., R. Arai \& I. Mabuchi, (2005) Small GTPase Rho5 is a functional homologue of Rho1, which controls cell shape and septation in fission yeast. FEBS Lett 579: 51815186.

Nakano, K., J. Imai, R. Arai, E. A. Toh, Y. Matsui \& I. Mabuchi, (2002) The small GTPase Rho3 and the diaphanous/formin For3 function in polarized cell growth in fission yeast. J Cell Sci 115: 4629-4639.

Nakano, K., T. Mutoh, R. Arai \& I. Mabuchi, (2003) The small GTPase Rho4 is involved in controlling cell morphology and septation in fission yeast. Genes Cells 8: 357-370.

Nakano, K., K. Satoh, A. Morimatsu, M. Ohnuma \& I. Mabuchi, (2001) Interactions among a fimbrin, a capping protein, and an actin-depolymerizing factor in organization of the fission yeast actin cytoskeleton. Mol Biol Cell 12: 3515-3526.

Nargang, F. E., K. P. Kunkele, A. Mayer, R. G. Ritzel, W. Neupert \& R. Lill, (1995) 'Sheltered disruption' of Neurospora crassa MOM22, an essential component of the mitochondrial protein import complex. EMBO J 14: 1099-1108.

Niccoli, T., M. Arellano \& P. Nurse, (2003) Role of Tea1p, Tea3p and Pom1p in the determination of cell ends in Schizosaccharomyces pombe. Yeast 20: 1349-1358.

Nishihama, R., J. H. Schreiter, M. Onishi, E. A. Vallen, J. Hanna, K. Moravcevic, M. F. Lippincott, H. Han, M. A. Lemmon, J. R. Pringle \& E. Bi, (2009) Role of Inn1 and its interactions with Hofl and Cyk3 in promoting cleavage furrow and septum formation in S. cerevisiae. J Cell Biol 185: 995-1012.

Ohkura, H., I. M. Hagan \& D. M. Glover, (1995) The conserved Schizosaccharomyces pombe kinase plo1, required to form a bipolar spindle, the actin ring, and septum, can drive septum formation in G1 and G2 cells. Genes Dev 9: 1059-1073.

Oliferenko, S., T. G. Chew \& M. K. Balasubramanian, (2009) Positioning cytokinesis. Genes Dev 23: 660-674.

Orlean, P., (1987) Two chitin synthases in Saccharomyces cerevisiae. J Biol Chem 262: 57325739.

Orr-Weaver, T. L. \& J. W. Szostak, (1983) Yeast recombination: the association between double-strand gap repair and crossing-over. Proc Natl Acad Sci U S A 80: 4417-4421.

Osman, M. A., J. B. Konopka \& R. A. Cerione, (2002) Iqg1p links spatial and secretion landmarks to polarity and cytokinesis. $J$ Cell Biol 159: 601-611.

Ozaki-Kuroda, K., Y. Yamamoto, H. Nohara, M. Kinoshita, T. Fujiwara, K. Irie \& Y. Takai, (2001) Dynamic localization and function of Bnilp at the sites of directed growth in Saccharomyces cerevisiae. Mol Cell Biol 21: 827-839.

Ozaki, K., K. Tanaka, H. Imamura, T. Hihara, T. Kameyama, H. Nonaka, H. Hirano, Y. Matsuura \& Y. Takai, (1996) Rom1p and Rom2p are GDP/GTP exchange proteins (GEPs) for the Rholp small GTP binding protein in Saccharomyces cerevisiae. EMBO J 15: 2196-2207.

Padte, N. N., S. G. Martin, M. Howard \& F. Chang, (2006) The cell-end factor pom1p inhibits midlp in specification of the cell division plane in fission yeast. Curr Biol 16: 24802487. 
Paoletti, A. \& F. Chang, (2000) Analysis of mid1p, a protein required for placement of the cell division site, reveals a link between the nucleus and the cell surface in fission yeast. Mol Biol Cell 11: 2757-2773.

Park, H. O. \& E. Bi, (2007) Central roles of small GTPases in the development of cell polarity in yeast and beyond. Microbiol Mol Biol Rev 71: 48-96.

Park, H. O., E. Bi, J. R. Pringle \& I. Herskowitz, (1997) Two active states of the Ras-related Bud1/Rsr1 protein bind to different effectors to determine yeast cell polarity. Proc Natl Acad Sci U S A 94: 4463-4468.

Park, H. O., J. Chant \& I. Herskowitz, (1993) BUD2 encodes a GTPase-activating protein for Bud1/Rsr1 necessary for proper bud-site selection in yeast. Nature 365: 269-274.

Park, H. O., P. J. Kang \& A. W. Rachfal, (2002) Localization of the Rsr1/Bud1 GTPase involved in selection of a proper growth site in yeast. J Biol Chem 277: 26721-26724.

Piekny, A., M. Werner \& M. Glotzer, (2005) Cytokinesis: welcome to the Rho zone. Trends Cell Biol 15: 651-658.

Piekny, A. J. \& M. Glotzer, (2008) Anillin is a scaffold protein that links RhoA, actin, and myosin during cytokinesis. Curr Biol 18: 30-36.

Pollard, T. D. \& J. Q. Wu, (2010) Understanding cytokinesis: lessons from fission yeast. Nat Rev Mol Cell Biol 11: 149-155.

Pringle, A. \& J. Taylor, (2002) The fitness of filamentous fungi. Trends Microbiol 10: 474481.

Pruyne, D., M. Evangelista, C. Yang, E. Bi, S. Zigmond, A. Bretscher \& C. Boone, (2002) Role of formins in actin assembly: nucleation and barbed-end association. Science 297: 612-615.

Rasmussen, C. G. \& N. L. Glass, (2005) A Rho-type GTPase, rho-4, is required for septation in Neurospora crassa. Eukaryot Cell 4: 1913-1925.

Rasmussen, C. G. \& N. L. Glass, (2007) Localization of RHO-4 indicates differential regulation of conidial versus vegetative septation in the filamentous fungus Neurospora crassa. Eukaryot Cell 6: 1097-1107.

Richman, T. J., M. M. Sawyer \& D. I. Johnson, (2002) Saccharomyces cerevisiae Cdc42p localizes to cellular membranes and clusters at sites of polarized growth. Eukaryot Cell 1: 458-468.

Rincon, S. A., B. Santos \& P. Perez, (2006) Fission yeast Rho5p GTPase is a functional paralogue of Rholp that plays a role in survival of spores and stationary-phase cells. Eukaryot Cell 5: 435-446.

Riquelme, M., G. Gierz \& S. Bartnicki-Garcia, (2000) Dynein and dynactin deficiencies affect the formation and function of the Spitzenkorper and distort hyphal morphogenesis of Neurospora crassa. Microbiology 146 ( Pt 7): 1743-1752.

Riquelme, M., R. W. Roberson, D. P. McDaniel \& S. Bartnicki-Garcia, (2002) The effects of ropy-1 mutation on cytoplasmic organization and intracellular motility in mature hyphae of Neurospora crassa. Fungal Genet Biol 37: 171-179.

Roberts-Galbraith, R. H. \& K. L. Gould, (2008) Stepping into the ring: the SIN takes on contractile ring assembly. Genes Dev 22: 3082-3088.

Roemer, T., K. Madden, J. Chang \& M. Snyder, (1996) Selection of axial growth sites in yeast requires Ax12p, a novel plasma membrane glycoprotein. Genes Dev 10: 777-793.

Roh, D. H., B. Bowers, M. Schmidt \& E. Cabib, (2002) The septation apparatus, an autonomous system in budding yeast. Mol Biol Cell 13: 2747-2759.

Rose, M. D., F. Winston \& P. Hieter, (1990) Methods in Yeast Genetics: A Laboratory Course Manual. Cold Spring Harbor, NY: Cold Spring Harbor Laboratory Press.

Rossman, K. L., C. J. Der \& J. Sondek, (2005) GEF means go: turning on RHO GTPases with guanine nucleotide-exchange factors. Nat Rev Mol Cell Biol 6: 167-180. 
Roumanie, O., H. Wu, J. N. Molk, G. Rossi, K. Bloom \& P. Brennwald, (2005) Rho GTPase regulation of exocytosis in yeast is independent of GTP hydrolysis and polarization of the exocyst complex. J Cell Biol 170: 583-594.

Sagot, I., S. K. Klee \& D. Pellman, (2002) Yeast formins regulate cell polarity by controlling the assembly of actin cables. Nat Cell Biol 4: 42-50.

Sambrook, J., E. F. Fritsch \& T. Maniatis, (1989) Molecular Cloning: A Laboratory Manual. New York: Cold Spring Harbor Laboratory Press.

Sanchez-Diaz, A., V. Marchesi, S. Murray, R. Jones, G. Pereira, R. Edmondson, T. Allen \& K. Labib, (2008) Inn1 couples contraction of the actomyosin ring to membrane ingression during cytokinesis in budding yeast. Nat Cell Biol 10: 395-406.

Sanders, S. L. \& I. Herskowitz, (1996) The BUD4 protein of yeast, required for axial budding, is localized to the mother/BUD neck in a cell cycle-dependent manner. $J$ Cell Biol 134: 413-427.

Santos, B., J. Gutierrez, T. M. Calonge \& P. Perez, (2003) Novel Rho GTPase involved in cytokinesis and cell wall integrity in the fission yeast Schizosaccharomyces pombe. Eukaryot Cell 2: 521-533.

Santos, B., A. B. Martin-Cuadrado, C. R. Vazquez de Aldana, F. del Rey \& P. Perez, (2005) Rho4 GTPase is involved in secretion of glucanases during fission yeast cytokinesis. Eukaryot Cell 4: 1639-1645.

Sawin, K. E., (2007) Cell division: mid-level management. Curr Biol 17: R93-95.

Schenkman, L. R., C. Caruso, N. Page \& J. R. Pringle, (2002) The role of cell cycle-regulated expression in the localization of spatial landmark proteins in yeast. $J$ Cell Biol 156: 829-841.

Schmidt, A. \& A. Hall, (2002) Guanine nucleotide exchange factors for Rho GTPases: turning on the switch. Genes Dev 16: 1587-1609.

Schmidt, M., A. Varma, T. Drgon, B. Bowers \& E. Cabib, (2003) Septins, under Cla4p regulation, and the chitin ring are required for neck integrity in budding yeast. Mol Biol Cell 14: 2128-2141.

Schmidt, S., M. Sohrmann, K. Hofmann, A. Woollard \& V. Simanis, (1997) The Spg1p GTPase is an essential, dosage-dependent inducer of septum formation in Schizosaccharomyces pombe. Genes Dev 11: 1519-1534.

Schmitz, H. P., A. Kaufmann, M. Kohli, P. P. Laissue \& P. Philippsen, (2006) From function to shape: a novel role of a formin in morphogenesis of the fungus Ashbya gossypii. Mol Biol Cell 17: 130-145.

Schuyler, S. C. \& D. Pellman, (2001) Search, capture and signal: games microtubules and centrosomes play. J Cell Sci 114: 247-255.

Shannon, K. B. \& R. Li, (1999) The multiple roles of Cyk1p in the assembly and function of the actomyosin ring in budding yeast. Mol Biol Cell 10: 283-296.

Shannon, K. B. \& R. Li, (2000) A myosin light chain mediates the localization of the budding yeast IQGAP-like protein during contractile ring formation. Curr Biol 10: 727-730.

Sharpless, K. E. \& S. D. Harris, (2002) Functional characterization and localization of the Aspergillus nidulans formin SEPA. Mol Biol Cell 13: 469-479.

Sheeman, B., P. Carvalho, I. Sagot, J. Geiser, D. Kho, M. A. Hoyt \& D. Pellman, (2003) Determinants of $S$. cerevisiae dynein localization and activation: implications for the mechanism of spindle positioning. Curr Biol 13: 364-372.

Si, H., D. Justa-Schuch, S. Seiler \& S. D. Harris, Regulation of Septum Formation by the Bud3-Rho4 GTPase Module in Aspergillus nidulans. Genetics. 2010 Feb. 23. [Epub ahead of print]. doi:10.1534/genetics.110.114165.

Simanis, V., (2003) Events at the end of mitosis in the budding and fission yeasts. $J$ Cell Sci 116: $4263-4275$. 
Slaughter, B. D., S. E. Smith \& R. Li, (2009) Symmetry breaking in the life cycle of the budding yeast. Cold Spring Harb Perspect Biol 1: a003384.

Sohrmann, M., C. Fankhauser, C. Brodbeck \& V. Simanis, (1996) The dmfl/mid1 gene is essential for correct positioning of the division septum in fission yeast. Genes Dev 10: 2707-2719.

Song, S. \& K. S. Lee, (2001) A novel function of Saccharomyces cerevisiae CDC5 in cytokinesis. J Cell Biol 152: 451-469.

Sudbery, P. E., (2001) The germ tubes of Candida albicans hyphae and pseudohyphae show different patterns of septin ring localization. Mol Microbiol 41: 19-31.

Tajadura, V., B. Garcia, I. Garcia, P. Garcia \& Y. Sanchez, (2004) Schizosaccharomyces pombe Rgf3p is a specific Rhol GEF that regulates cell wall beta-glucan biosynthesis through the GTPase Rho1p. J Cell Sci 117: 6163-6174.

Tasto, J. J., J. L. Morrell \& K. L. Gould, (2003) An anillin homologue, Mid2p, acts during fission yeast cytokinesis to organize the septin ring and promote cell separation. J Cell Biol 160: 1093-1103.

Tcherkezian, J. \& N. Lamarche-Vane, (2007) Current knowledge of the large RhoGAP family of proteins. Biol Cell 99: 67-86.

Tolic-Norrelykke, I. M., L. Sacconi, C. Stringari, I. Raabe \& F. S. Pavone, (2005) Nuclear and division-plane positioning revealed by optical micromanipulation. Curr Biol 15: 1212-1216.

Tolliday, N., N. Bouquin \& R. Li, (2001) Assembly and regulation of the cytokinetic apparatus in budding yeast. Curr Opin Microbiol 4: 690-695.

Tolliday, N., L. VerPlank \& R. Li, (2002) Rho1 directs formin-mediated actin ring assembly during budding yeast cytokinesis. Curr Biol 12: 1864-1870.

Tong, Z., X. D. Gao, A. S. Howell, I. Bose, D. J. Lew \& E. Bi, (2007) Adjacent positioning of cellular structures enabled by a Cdc42 GTPase-activating protein-mediated zone of inhibition. J Cell Biol 179: 1375-1384.

Tran, P. T., L. Marsh, V. Doye, S. Inoue \& F. Chang, (2001) A mechanism for nuclear positioning in fission yeast based on microtubule pushing. $J$ Cell Biol 153: 397-411.

Vallen, E. A., J. Caviston \& E. Bi, (2000) Roles of Hoflp, Bnilp, Bnrlp, and myolp in cytokinesis in Saccharomyces cerevisiae. Mol Biol Cell 11: 593-611.

Vanni, C., C. Ottaviano, F. Guo, M. Puppo, L. Varesio, Y. Zheng \& A. Eva, (2005) Constitutively active Cdc42 mutant confers growth disadvantage in cell transformation. Cell Cycle 4: 1675-1682.

Veith, D., N. Scherr, V. P. Efimov \& R. Fischer, (2005) Role of the spindle-pole-body protein ApsB and the cortex protein ApsA in microtubule organization and nuclear migration in Aspergillus nidulans. J Cell Sci 118: 3705-3716.

Versele, M. \& J. Thorner, (2004) Septin collar formation in budding yeast requires GTP binding and direct phosphorylation by the PAK, Cla4. J Cell Biol 164: 701-715.

Vogt, N., (2008) Governing fungal polar cell extension: Analysis of Rho GTPase and NDR kinase signalling in Neurospora crassa. Dissertation, Georg-August-Universität Göttingen.

Vogt, N. \& S. Seiler, (2008) The RHO1-specific GTPase-activating protein LRG1 regulates polar tip growth in parallel to Ndr kinase signaling in Neurospora. Mol Biol Cell 19: 4554-4569.

Vollmer, S. J. \& C. Yanofsky, (1986) Efficient cloning of genes of Neurospora crassa. Proc Natl Acad Sci U S A 83: 4869-4873.

Wachtler, V., Y. Huang, J. Karagiannis \& M. K. Balasubramanian, (2006) Cell cycledependent roles for the FCH-domain protein Cdc15p in formation of the actomyosin ring in Schizosaccharomyces pombe. Mol Biol Cell 17: 3254-3266. 
Walther, A. \& J. Wendland, (2003) Septation and cytokinesis in fungi. Fungal Genetics and Biology 40: 187-196.

Wang, J., H. Hu, S. Wang, J. Shi, S. Chen, H. Wei, X. Xu \& L. Lu, (2009) The important role of actinin-like protein (AcnA) in cytokinesis and apical dominance of hyphal cells in Aspergillus nidulans. Microbiology 155: 2714-2725.

Warenda, A. J. \& J. B. Konopka, (2002) Septin function in Candida albicans morphogenesis. Mol Biol Cell 13: 2732-2746.

Wedlich-Soldner, R., S. Altschuler, L. Wu \& R. Li, (2003) Spontaneous cell polarization through actomyosin-based delivery of the Cdc42 GTPase. Science 299: 1231-1235.

Wendland, J., (2003) Analysis of the landmark protein Bud3 of Ashbya gossypii reveals a novel role in septum construction. EMBO Rep 4: 200-204.

Wendland, J., Y. Ayad-Durieux, P. Knechtle, C. Rebischung \& P. Philippsen, (2000) PCRbased gene targeting in the filamentous fungus Ashbya gossypii. Gene 242: 381-391.

Wendland, J. \& P. Philippsen, (2001) Cell polarity and hyphal morphogenesis are controlled by multiple rho-protein modules in the filamentous ascomycete Ashbya gossypii. Genetics 157: 601-610.

Wendland, J. \& P. Philippsen, (2002) An IQGAP-related protein, encoded by AgCYK1, is required for septation in the filamentous fungus Ashbya gossypii. Fungal Genet Biol 37: 81-88.

Wennerberg, K., K. L. Rossman \& C. J. Der, (2005) The Ras superfamily at a glance. J Cell Sci 118: 843-846.

Westfall, P. J. \& M. Momany, (2002) Aspergillus nidulans septin AspB plays pre- and postmitotic roles in septum, branch, and conidiophore development. Mol Biol Cell 13: 110-118.

Wolfe, B. A. \& K. L. Gould, (2005) Split decisions: coordinating cytokinesis in yeast. Trends Cell Biol 15: 10-18.

Wolkow, T. D., S. D. Harris \& J. E. Hamer, (1996) Cytokinesis in Aspergillus nidulans is controlled by cell size, nuclear positioning and mitosis. J Cell Sci 109 ( Pt 8): 21792188.

Woodcock, D. M., P. J. Crowther, J. Doherty, S. Jefferson, E. DeCruz, M. Noyer-Weidner, S. S. Smith, M. Z. Michael \& M. W. Graham, (1989) Quantitative evaluation of Escherichia coli host strains for tolerance to cytosine methylation in plasmid and phage recombinants. Nucleic Acids Res 17: 3469-3478.

Wortman, J. R., J. M. Gilsenan, V. Joardar, J. Deegan, J. Clutterbuck, M. R. Andersen, D. Archer, M. Bencina, G. Braus, P. Coutinho, H. von Dohren, J. Doonan, A. J. Driessen, P. Durek, E. Espeso, E. Fekete, M. Flipphi, C. G. Estrada, S. Geysens, G. Goldman, P. W. de Groot, K. Hansen, S. D. Harris, T. Heinekamp, K. Helmstaedt, B. Henrissat, G. Hofmann, T. Homan, T. Horio, H. Horiuchi, S. James, M. Jones, L. Karaffa, Z. Karanyi, M. Kato, N. Keller, D. E. Kelly, J. A. Kiel, J. M. Kim, I. J. van der Klei, F. M. Klis, A. Kovalchuk, N. Krasevec, C. P. Kubicek, B. Liu, A. Maccabe, V. Meyer, P. Mirabito, M. Miskei, M. Mos, J. Mullins, D. R. Nelson, J. Nielsen, B. R. Oakley, S. A. Osmani, T. Pakula, A. Paszewski, I. Paulsen, S. Pilsyk, I. Pocsi, P. J. Punt, A. F. Ram, Q. Ren, X. Robellet, G. Robson, B. Seiboth, P. van Solingen, T. Specht, J. Sun, N. Taheri-Talesh, N. Takeshita, D. Ussery, P. A. vanKuyk, H. Visser, P. J. van de Vondervoort, R. P. de Vries, J. Walton, X. Xiang, Y. Xiong, A. P. Zeng, B. W. Brandt, M. J. Cornell, C. A. van den Hondel, J. Visser, S. G. Oliver \& G. Turner, (2009) The 2008 update of the Aspergillus nidulans genome annotation: a community effort. Fungal Genet Biol 46 Suppl 1: S2-13.

Wu, J. Q., J. Bahler \& J. R. Pringle, (2001) Roles of a fimbrin and an alpha-actinin-like protein in fission yeast cell polarization and cytokinesis. Mol Biol Cell 12: 1061-1077. 
Wu, J. Q., J. R. Kuhn, D. R. Kovar \& T. D. Pollard, (2003) Spatial and temporal pathway for assembly and constriction of the contractile ring in fission yeast cytokinesis. Dev Cell 5: $723-734$.

Yeh, E., C. Yang, E. Chin, P. Maddox, E. D. Salmon, D. J. Lew \& K. Bloom, (2000) Dynamic positioning of mitotic spindles in yeast: role of microtubule motors and cortical determinants. Mol Biol Cell 11: 3949-3961.

Yeong, F. M., (2005) Severing all ties between mother and daughter: cell separation in budding yeast. Mol Microbiol 55: 1325-1331.

Yoshida, S., K. Asakawa \& A. Toh-e, (2002) Mitotic exit network controls the localization of Cdc14 to the spindle pole body in Saccharomyces cerevisiae. Curr Biol 12: 944-950.

Yoshida, S. \& A. Toh-e, (2001) Regulation of the localization of Dbf2 and mob1 during cell division of Saccharomyces cerevisiae. Genes Genet Syst 76: 141-147.

Zahner, J. E., H. A. Harkins \& J. R. Pringle, (1996) Genetic analysis of the bipolar pattern of bud site selection in the yeast Saccharomyces cerevisiae. Mol Cell Biol 16: 18571870.

Zheng, Y., A. Bender \& R. A. Cerione, (1995) Interactions among proteins involved in budsite selection and bud-site assembly in Saccharomyces cerevisiae. J Biol Chem 270: 626-630. 


\section{Acknowledgements}

First of all I would like to thank Prof. Dr. Gerhard Braus for giving me the opportunity to conduct my Ph.D. research in his department. I highly appreciate the great working conditions there.

I am very grateful to Dr. Stephan Seiler for his supervision, endless support and friendly discussions during the course of my work. I also thank him for giving me the opportunity to participate in national and international conferences.

Furthermore, I would like to thank Prof. Dr. Stefanie Pöggeler for being the co-referent of this thesis. For providing plasmids, protocols and helpful hints I am grateful to the whole "Pöggeler" group, especially to Yasmine Bernhards.

Special thanks go to Yvonne Heilig for her contribution to this work by her diploma thesis research. You did a fantastic job!

Further thanks go the whole department for the great working atmosphere, the support in experimental questions and the friendly discussions, especially during the lunch breaks. In this context, I also wish to thank Martin Christmann and Rebekka Harting for providing Aspergillus cDNA.

Special thanks go to all present and former members of the "007" lab: Josie, Inga, Nico, Thomas, Immo, Bine, Anne and Yvonne. I would like to thank them for their endless helpfulness, the fruitful discussions, their support especially after setbacks and the good atmosphere in the lab (at least during most of the time;-)). Thank you for being such great colleagues!

My deepest thanks go to my friends, my family, and especially my husband Sebastian for accompanying me through all the ups and downs during this work. Without your endless support the completion of this thesis would not have been possible.

\section{THANK YOU!}




\section{CURRICULUM VITAE}

\section{Daniela Justa-Schuch}

Anschrift:

Geburtsdatum:

Geburtsort:

Staatsangehörigkeit:

Januar 2006 - März 2010

November 2005 - Dezember 2005

Oktober 2000 - Juli 2005

August 1993 - Juli 2000

$1991-1993$

$1987-1991$
Am Pfingstanger 53

37075 Göttingen

04.06.1981

Quakenbrück

deutsch

Doktorarbeit am Institut für Mikrobiologie und Genetik der Georg-August-Universität Göttingen

in der Abteilung für molekulare Mikrobiologie und Genetik (Prof. G. Braus)

Thema: Regulation of septum formation by RHO4 GTPase signalling in Neurospora crassa

Anstellung als wissenschaftliche Hilfskraft am Institut für Mikrobiologie und Genetik der Georg-August-Universität Göttingen

Biologiestudium an der Ruprecht-Karls Universität Heidelberg

Abschluss: Diplom-Biologe

Hauptfach: Molekularbiologie

1. Nebenfach: Zellbiologie

2. Nebenfach: Biochemie

Thema der Diplomarbeit: „Rolle der viralen Onkogene E6 und E7 des humanen Papillomvirus in der genomischen Instabilität humaner Keratinozyten“

Gymnasium Bersenbrück, Bersenbrück

Orientierungsstufe, Bersenbrück

Grundschule, Bersenbrück 